\title{
Notes on Conservation Laws, Equations of Motion of Matter, and Particle Fields in Lorentzian and Teleparallel de Sitter Space-Time Structures
}

\author{
Waldyr A. Rodrigues and Samuel A. Wainer \\ IMECC, UNICAMP, Campinas, SP, Brazil \\ Correspondence should be addressed to Waldyr A. Rodrigues; walrod@ime.unicamp.br
}

Received 28 September 2015; Revised 9 November 2015; Accepted 2 December 2015

Academic Editor: Claudio Dappiaggi

Copyright (C) 2016 W. A. Rodrigues and S. A. Wainer. This is an open access article distributed under the Creative Commons Attribution License, which permits unrestricted use, distribution, and reproduction in any medium, provided the original work is properly cited.

\begin{abstract}
We discuss the physics of interacting fields and particles living in a de Sitter Lorentzian manifold (dSLM), a submanifold of a 5-dimensional pseudo-Euclidean (5dPE) equipped with a metric tensor inherited from the metric of the 5dPE space. The dSLM is naturally oriented and time oriented and is the arena used to study the energy-momentum conservation law and equations of motion for physical systems living there. Two distinct de Sitter space-time structures $M^{\mathrm{dSL}}$ and $M^{\mathrm{dSTP}}$ are introduced given dSLM, the first equipped with the Levi-Civita connection of its metric field and the second with a metric compatible parallel connection. Both connections are used only as mathematical devices. Thus, for example, $M^{\mathrm{dSL}}$ is not supposed to be the model of any gravitational field in the General Relativity Theory (GRT). Misconceptions appearing in the literature concerning the motion of free particles in dSLM are clarified. Komar currents are introduced within Clifford bundle formalism permitting the presentation of Einstein equation as a Maxwell like equation and proving that in GRT there are infinitely many conserved currents. We prove that in GRT even when the appropriate Killing vector fields exist it is not possible to define a conserved energy-momentum covector as in special relativistic theories.
\end{abstract}

\section{Introduction}

In this paper we study some aspects of Physics of fields living and interacting in a manifold $M=\operatorname{SO}(1,4) / \mathrm{SO}(1,3) \simeq$ $\mathbb{R} \times S^{3}$. We introduce two different geometrical space-time structures that we can form starting from the manifold $M$ which is supposed to be a vector manifold, that is, a submanifold of $(\stackrel{\circ}{M}, \stackrel{\mathrm{g}}{\mathrm{g}})$ with $\stackrel{M}{M}=\mathbb{R}^{5}$ and $\stackrel{\circ}{\mathrm{g}}$ a metric of signature $(1,4)$. If $\mathbf{i}: M \rightarrow \stackrel{M}{\text { is }}$ is the inclusion map the structures that will be studied are the Lorentzian de Sitter space-time $M^{\mathrm{dSL}}=\left(M, \mathbf{g}, \mathbf{D}, \tau_{\mathbf{g}}, \uparrow\right)$ and teleparallel de Sitter space-time $M^{\mathrm{dSTP}}=\left(M, \mathbf{g}, \boldsymbol{\nabla}, \tau_{\mathbf{g}}, \uparrow\right)$, where $\mathbf{g}=\mathbf{i}^{*} \mathrm{~g}, \mathbf{D}$ is the Levi-Civita connection of $\mathbf{g}$, and $\boldsymbol{\nabla}$ is a metric compatible teleparallel connection (see Section 4.1). Our main objective is the following: taking $(M, \mathbf{g})$ as the arena where physical fields live and interact, how do we formulate conservation laws of energy-momentum and angular momentum for the system of physical fields? In order to give a meaningful meaning to this question we recall the fact that in Lorentzian space-time structures that are models of gravitational fields in the GRT there are no genuine conservation laws of energymomentum (and also angular momentum) for a closed system of fields and moreover there are no genuine energymomentum and angular momentum conservation laws for the system consisting of nongravitational plus the gravitational field. We discuss in Section 2.1 a pure mathematical result, namely, when there exist some conserved currents $\mathscr{F}_{V} \in \sec T^{*} M$ in a Lorentzian space-time associated with a tensor field $\mathbb{W} \in \sec T_{1}^{1} M$ and a vector field $\mathbf{V} \in \sec T M$. In Section 2.2 we briefly recall how a conserved energymomentum tensor for the matter fields is constructed in Special Relativity theories and how in that theory it is possible to construct a conserved energy-momentum covector ${ }^{1}$ for the matter fields. After that we recall that in GRT we have a covariantly "conserved" energy-momentum tensor 
$\mathbf{T} \in \sec T_{1}^{1} M$ (i.e., $\mathbf{D} \cdot \mathbf{T}=0$ ) and so, using the results of Section 2.1, we can immediately construct conserved currents when the Lorentzian space-time modelling the gravitational field generated by $\mathbf{T}$ possesses Killing vector fields. However, we show that it is not possible in general in GRT even when some special conserved currents exist (associated with one timelike and three spacelike Killing vector fields) to build a conserved covector for the system of fields, as it is the case in special relativistic theories. Immediately after showing that we ask the following question:

Is it necessary to have Killing vector fields in a Lorentzian space-time modelling a given gravitational field in order to be possible to construct conserved currents?

Well, we show that the answer is no. In GRT there are an infinite number of conserved currents. This is shown in Section $2.4^{2}$ where we introduce the so-called Komar currents in a Lorentzian space-time modelling a gravitational field generated by a given (symmetric) energy-momentum tensor $\mathbf{T}$ and we show how any diffeomorphism associated with a one-parameter group generated by a vector field $\mathbf{A}$ leads to a conserved current. We show moreover using the Clifford bundle formalism recalled in Appendix A that $F=$ $d A \in \sec \bigwedge^{2} T^{*} M$, where $A=\mathbf{g}(\mathbf{A},) \in \sec \bigwedge^{1} T^{*} M$ satisfy (with $\partial$ denoting the Dirac operator acting on sections of the Clifford bundle of differential forms) a Maxwell-like equation $\partial F=\mathbf{J}_{A}$ (equivalent to $d F=0$ and $\underset{\mathbf{g}}{\delta}=-\mathbf{J}_{A}$ ). The explicit form of $\mathbf{J}_{A}$ as a function of the energy-momentum tensor is derived (see (50)) together with its scalar invariant. We establish that ${ }^{3} \partial F=\mathbf{J}_{A}$ encode the contents of Einstein equation. We show moreover that even if we can get four conserved currents given one timelike and three spacelike vector fields and thus get four scalar invariants, these objects cannot be associated with the components of a momentum covector $^{4}$ for the system of fields producing the energymomentum tensor $\mathbf{T}$. We also give the form of $\mathbf{J}_{A}$ when $\mathbf{A}$ is a Killing vector field and emphasize that even if the Lorentzian space-time under consideration has one timelike and three spacelike Killing vector fields we cannot find a conserved momentum covector for the system of fields.

This paper has several appendices necessary for a perfect intelligibility of the results in the main text. Thus it is opportune to describe what is there and where their contents are used in the main text ${ }^{5}$. To start, in Appendix A, we briefly recall the main results of the Clifford bundle formalism used in this paper which permits one to understand how to arrive at the equation $\partial F=\mathbf{J}_{A}$ in Section $2.2^{6}$. Lie derivatives and variations of tensor fields are discussed in Appendix B. In Appendix C.1 we derive from the Lagrangian formalism conserved currents for fields living in a general Lorentzian spacetime structure and the corresponding generalized covariant energy-momentum "conservation" law. We compare these results in Appendix C.2 with the analogus ones for field theories in special relativistic theories where the Lorentzian space-time structure is Minkowski space-time. We show that despite the fact that we can derive conserved quantities for fields living and interacting in $M^{\mathrm{dSL}}$ we cannot define in this structure a genuine energy-momentum conserved covector for the system of fields as it is the case in Minkowski spacetime. A legitimate energy-momentum covector for the system of fields living in $(M=\mathrm{SO}(1,4) / \mathrm{SO}(1,3)$, g) exists only in the teleparallel structure $M^{\mathrm{dSTP}}$. This is discussed in Section 5.2 after recalling the Lie algebra and the Casimir invariants of the Lie algebra of de Sitter group in Section 5.1. In Appendix D we derive for completeness and to insert Remark D. 3 the so-called covariant energy-momentum conservation law in GRT. Appendix E recalls the intrinsic definition of relative tensors and their covariant derivatives. Appendix F presents proofs of some identities used in the main text.

As we already said the main objective of this paper is to discuss the Physics of interacting fields in de Sitter spacetime structures $M^{\mathrm{dSL}}$ and $M^{\mathrm{dSTP}}$. In particular we want also to clarify some misunderstandings concerning the roles of geodesics in $M^{\mathrm{dSL}}$. So, in Section 3, we briefly recall the conformal representation of the de Sitter space-time structure $M^{\mathrm{dSL}}$ and prove that the one timelike and the three spacelike "translation" Killing vector fields of $(M, \mathbf{g})$ define a basis for almost all $M$. With this result we show in Section 4 that the method used in [1] to obtain the curves which minimizes or maximizes the length function of timelike curves in de Sitter space-time with the result that these curves are not geodesics is equivocated, since those authors use constrained variations instead of arbitrary variations of the length function. Even more, the equation obtained from the constrained variation in [1] is according to our view wrongly interpreted in its mathematical (and physical) contents. Indeed, using some of the results of Section 5.2 and the results of Section 6 which briefly recall Papapetrou's classical results [2] deriving the equation of motion of a probe single-pole particle in GRT, we show in Section 7 that contrary to the authors statement in [1] it is not true that the equation of motion of a singlepole obtained from a method similar to Papapetrou's in [2] but using the generalized energy-momentum tensor of matter fields in $M^{\mathrm{dSL}}$ gives an equation of motion different from the geodesic equation in $M^{\mathrm{dSL}}$ and in agreement with the one they derived from his constrained variation method. Indeed, we prove that from the equation describing the motion of a single-pole the geodesic equation follows automatically.

\section{Preliminaries}

Let $\left(M, \mathbf{g}, D, \tau_{\mathbf{g}}, \uparrow\right)$ be a general Lorentzian space-time. Let $\mathcal{U} \subseteq M$ be an open set covered by coordinates $\left\{x^{\mu}\right\}$. Let $\left\{e_{\mu}=\partial_{\mu}\right\}$ be a basis of $T \mathscr{U}$ and $\left\{\boldsymbol{\vartheta}^{\mu}=d x^{\mu}\right\}$ the basis of $T^{*} \mathcal{U}$ dual to the basis $\left\{\partial_{\mu}\right\}$; that is, $\boldsymbol{\vartheta}^{\mu}\left(\partial_{\nu}\right)=\delta_{\nu}^{\mu}$. We denote by $\mathrm{g}$ a metric of the cotangent bundle such that if $\mathbf{g}=g_{\mu \nu} \boldsymbol{\vartheta}^{\mu} \otimes \boldsymbol{\vartheta}^{\nu}$, then $g=g^{\mu \nu} \partial_{\mu} \otimes \partial_{\nu}$ with $g^{\mu \rho} g_{\rho \nu}=\delta_{\nu}^{\mu}$. We introduce also $\left\{\partial^{\mu}\right\}$ and $\left\{\boldsymbol{\vartheta}_{\mu}\right\}$, respectively, as the reciprocal bases of $\left\{\partial_{\mu}\right\}$ and $\left\{\boldsymbol{\vartheta}^{\mu}\right\}$; that is, we have

$$
\begin{gathered}
\mathbf{g}\left(\partial_{v}, \partial^{\mu}\right)=\delta_{v}^{\mu}, \\
\mathbf{g}\left(\boldsymbol{\vartheta}^{\mu}, \boldsymbol{\vartheta}_{v}\right)=\delta_{v}^{\mu} .
\end{gathered}
$$

Next we introduce in TU the tetrad basis $\left\{\mathbf{e}_{\alpha}=h_{\alpha}^{\mu} \partial_{\mu}\right\}$ and in $T^{*} \mathcal{U}$ the cotetrad basis $\left\{\gamma^{\alpha}=h_{\mu}^{\alpha} \gamma^{\mu}\right\}$ which are dual 
basis. We introduce moreover the bases $\left\{\mathbf{e}^{\alpha}\right\}$ and $\left\{\boldsymbol{\gamma}_{\alpha}\right\}$ as the reciprocal bases of $\left\{\mathbf{e}_{\alpha}\right\}$ and $\left\{\gamma^{\alpha}\right\}$ satisfying

$$
\begin{aligned}
& \mathbf{g}\left(\mathbf{e}_{\alpha}, \mathbf{e}^{\beta}\right)=\delta_{\alpha}^{\beta}, \\
& \mathrm{g}\left(\boldsymbol{\gamma}^{\beta}, \gamma_{\alpha}\right)=\delta_{\alpha}^{\beta} .
\end{aligned}
$$

Moreover recall that it is

$$
\begin{aligned}
& \mathbf{g}=\eta_{\alpha \beta} \gamma^{\alpha} \otimes \gamma^{\beta}=\eta^{\alpha \beta} \gamma_{\alpha} \otimes \gamma_{\beta}, \\
& \mathbf{g}=\eta^{\alpha \beta} \mathbf{e}_{\alpha} \otimes \mathbf{e}_{\beta}=\eta_{\alpha \beta} \mathbf{e}^{\alpha} \otimes \mathbf{e}^{\beta} .
\end{aligned}
$$

2.1. The Currents $\mathscr{J}_{V}$ and $\mathscr{J}_{K}$. Let $\mathbf{W}=W^{\alpha \beta} \mathbf{e}_{\alpha} \otimes \mathbf{e}_{\beta} \in$ $\sec T_{2}^{0} M$ with $W^{\alpha \beta}=W^{\beta \alpha}$ and $\check{\mathbf{W}}=W_{\alpha \beta} \gamma^{\alpha} \otimes \gamma^{\beta} \in \sec T_{0}^{2} M$, $W_{\alpha \beta}=\eta_{\alpha \varsigma} \eta_{\beta \tau} W^{\varsigma \tau}$, and $\mathbb{W}=W_{\beta}^{\alpha} \gamma^{\beta} \otimes \mathbf{e}_{\alpha} \in \sec T_{1}^{1} M$. For the applications we have in mind we will say that $\mathbf{W}, \check{\mathbf{W}}$, and $\mathbb{W}$ are physically equivalent.

Note that $\mathbb{W}$ (an example of an extensor field ${ }^{7}$ ) is such that

$$
\begin{gathered}
\mathbb{W}: \sec \bigwedge^{1} T^{*} M \longrightarrow \sec \bigwedge^{1} T^{*} M, \\
\mathbb{W}(V)=V_{\alpha} W_{\beta}^{\alpha} \gamma^{\beta} .
\end{gathered}
$$

Define the divergence of $\mathbb{W}$ as the 1-form field

$$
\mathrm{D} \cdot \mathbb{W}:=\left(\mathbf{D}_{\alpha} W_{\beta}^{\alpha}\right) \gamma^{\beta}
$$

where

$$
\mathbf{D}_{\alpha} W_{\beta}^{\alpha}:=\left(\mathbf{D}_{\mathbf{e}_{\alpha}} \mathbb{W}\right)_{\beta}^{\alpha}=\mathbf{e}_{\alpha}\left(W_{\beta}^{\alpha}\right)+\Gamma_{\alpha \iota}^{\alpha} W_{\beta}^{\iota}-\Gamma_{\alpha \beta}^{l} W_{\imath}^{\alpha} .
$$

Moreover, introduce the 1 -form fields

$$
\mathscr{W}^{\beta}:=W^{\alpha \beta} \gamma_{\alpha} \in \sec \bigwedge^{1} T^{*} M .
$$

Remark 1. Take notice for the developments that follow that the Hodge coderivative of the 1 -form fields $\mathscr{W}^{\beta}$ is as follows (see Appendix):

$$
\begin{aligned}
\underset{\mathbf{g}}{\delta \mathscr{W}^{\beta}} & =-\gamma^{\kappa}{ }_{\lrcorner} \mathbf{D}_{\mathbf{e}_{\kappa}}\left(W^{\alpha \beta} \gamma_{\alpha}\right) \\
& \left.\left.=-\mathbf{e}_{\kappa}\left(W^{\alpha \beta}\right) \gamma^{\kappa}\right\lrcorner \gamma_{\alpha}-W^{\alpha \beta} \Gamma_{\kappa \alpha}^{l} \gamma^{\kappa}\right\lrcorner \gamma_{\iota} \\
& =-\mathbf{e}_{\alpha}\left(W^{\alpha \beta}\right)-W^{\alpha \beta} \Gamma_{\kappa \alpha}^{\kappa} .
\end{aligned}
$$

So, $\mathbf{D} \bullet \mathbb{W}=0$ does not imply that $\underset{\mathbf{g}}{\delta \mathscr{W}^{\beta}}=0$.

Now, given a vector field $\mathbf{V}=V^{\alpha} \mathbf{e}_{\alpha}$ and the physically equivalent covector field $V=V^{\alpha} \gamma_{\alpha}$, define the current

$$
\mathscr{J}_{V}=V^{\alpha} \mathscr{W}_{\alpha} \in \sec \bigwedge^{1} T^{*} M \hookrightarrow \sec \mathscr{C} \ell(M, g)
$$

Of course, writing

$$
\mathscr{J}_{V}=\mathscr{J}_{\beta} \gamma^{\beta}
$$

we have

$$
\mathscr{J}_{\beta}=V^{\alpha} W_{\alpha \beta} \text {. }
$$

Recalling (see Appendix A) that $\star 1=\tau_{\mathrm{g}}$, define

$$
\mathfrak{T}=W_{\mathrm{g}}^{\alpha \beta} \underset{\star}{*} 1=W^{\alpha \beta} \tau_{\mathbf{g}} \in \sec \bigwedge^{4} T^{*} M \hookrightarrow \mathscr{C l}(M, \mathrm{~g})
$$

Then, we have, with $\partial$ denoting the Dirac operator,

$$
\begin{aligned}
d \underset{\mathbf{g}}{\star} \mathscr{J}_{V} & =\partial \wedge \underset{\mathbf{g}}{\star} \mathscr{J}_{V}=\gamma^{\alpha} \wedge\left(\mathbf{D}_{\mathbf{e}_{\alpha}} \underset{\mathbf{g}}{\star} \mathscr{J}_{V}\right) \\
& =\mathbf{D}_{\mathbf{e}_{\alpha}}\left(\gamma^{\alpha} \wedge \underset{\mathbf{g}}{\star} \mathscr{J}_{V}\right)-\mathbf{D}_{\mathbf{e}_{\alpha}} \gamma^{\alpha} \wedge \underset{\mathbf{g}}{\star} \mathscr{J}_{V} .
\end{aligned}
$$

Taking into account that $\left.\gamma^{\alpha} \wedge \underset{\mathrm{g}}{\star \mathscr{J}_{V}}=\underset{\mathrm{g}}{\star}\left(\boldsymbol{\gamma}^{\alpha}\right\lrcorner \mathscr{J}_{V}\right)$ and $\left.\mathbf{D}_{\mathbf{e}_{\alpha}} \gamma^{\alpha} \wedge \underset{\mathbf{g}}{\star} \mathscr{J}_{V}=\underset{\mathbf{g}}{\star}\left(\mathbf{D}_{\mathbf{e}_{\alpha}} \gamma^{\alpha}\right\lrcorner \mathscr{J}_{V}\right)$ we can write (13) as

$$
\begin{aligned}
& d \stackrel{g_{\mathrm{g}}}{\mathscr{J}_{V}} \\
& =\left(\mathbf{e}_{\alpha}\left(V^{\kappa}\right) W_{\kappa}^{\alpha}+V^{\kappa} \mathbf{e}_{\alpha}\left(W_{\kappa}^{\alpha}\right)+\Gamma_{\cdot \alpha \beta}^{\alpha \cdot} V^{\kappa} W_{\kappa}^{\beta}\right) \tau_{\mathbf{g}} .
\end{aligned}
$$

Also, we can easily verify from (5) that

$$
\begin{aligned}
\stackrel{\star}{\mathbf{g}} & {[(\mathbf{D} \cdot \mathbb{W})(\mathbf{V})]=[(\mathbf{D} \cdot \mathbb{W})(\mathbf{V})] \tau_{\mathbf{g}} } \\
& =\left(V^{\kappa} \mathbf{e}_{\alpha}\left(W_{\kappa}^{\alpha}\right)+\Gamma_{\cdot \alpha l}^{\alpha \cdot} W_{\kappa}^{\iota} V^{\kappa}-\Gamma_{\cdot \alpha \kappa}^{\iota \cdot} W_{l}^{\alpha} V^{\kappa}\right) \tau_{\mathbf{g}} .
\end{aligned}
$$

Now, let $£$ be the (standard) Lie derivative operator. Let us evaluate the product of $\mathfrak{E}_{\mathbf{V}} \mathbf{g}\left(\mathbf{e}_{\alpha}, \mathbf{e}_{\beta}\right)$ by $\mathfrak{T}$; that is,

$$
\left(\mathfrak{E}_{\mathbf{V}} \mathbf{g}\left(\mathbf{e}_{\alpha}, \mathbf{e}_{\beta}\right)\right) \mathfrak{I} \in \sec \bigwedge^{4} T^{*} M
$$

From Cartan magical formula we get

$$
\begin{aligned}
\mathfrak{E}_{\mathrm{V}} \gamma^{\alpha} & =d\left(V^{\alpha}\right)+V_{\lrcorner}\left(\partial \wedge \gamma^{\alpha}\right) \\
& =\mathbf{e}_{\iota}\left(V^{\alpha}\right) \boldsymbol{\gamma}^{\iota}-V^{\varsigma} \Gamma_{\cdot \zeta \iota}^{\alpha \cdot \cdot} \gamma^{\iota}+V^{\varsigma} \Gamma_{\cdot \iota \zeta}^{\alpha \cdot \cdot} \gamma^{\iota} .
\end{aligned}
$$

Then,

$$
\begin{aligned}
& {\left[\mathfrak{E}_{\mathrm{V}} \mathbf{g}\left(\mathbf{e}_{\alpha}, \mathbf{e}_{\beta}\right)\right] \mathfrak{T}} \\
& \quad=\left[\left(\eta_{\iota \mathcal{K}} \mathfrak{E}_{\mathrm{V}} \gamma^{\iota} \otimes \gamma^{\kappa}+\eta_{\iota \kappa} \gamma^{\iota} \otimes \mathfrak{E}_{\mathrm{V}} \gamma^{\kappa}\right)\left(\mathbf{e}_{\alpha}, \mathbf{e}_{\beta}\right)\right] \mathfrak{T} \\
& \quad=\left[2 \mathbf{e}_{\kappa}\left(V_{\iota}\right) W^{\kappa \iota}+2 V^{\varsigma} \Gamma_{\cdot \iota \varsigma}^{\kappa \cdot} W_{\kappa}^{\iota}\right] \mathfrak{T}
\end{aligned}
$$

and we get from (14), (15), and (18) the important identity [3]

$$
\left(\mathfrak{E}_{\mathbf{V}} \mathbf{g}\left(\mathbf{e}_{\alpha}, \mathbf{e}_{\beta}\right)\right) \mathfrak{T}=2 d \star \mathscr{J}_{V}-2 \underset{\mathrm{g}}{2}[(\mathbf{D} \bullet \mathbb{W})(\mathbf{V})] .
$$

From (19) we see that if $\mathbf{V}$ is a conformal Killing vector field, that is, $\mathfrak{E}_{\mathbf{V}} \mathbf{g}\left(\mathbf{e}_{\alpha}, \mathbf{e}_{\beta}\right)=2 \lambda \eta_{\alpha \beta}$, we have

$$
\underset{\mathrm{g}}{\star \lambda} \operatorname{tr} \mathbb{W}=d \underset{\mathrm{g}}{\star} \mathscr{J}_{V}-\star[(\mathbf{D} \cdot \mathbb{W})(\mathbf{V})],
$$

where $\operatorname{tr} \mathbb{W}$ is the trace of the matrix with entries $W_{\beta}^{\alpha}$. 


\subsection{Conserved Currents Associated with a Covariantly Conserved W}

Definition 2. One says that $\mathbb{W}$ is "covariantly conserved" if

$$
\mathbf{D} \cdot \mathbb{W}=0 \text {. }
$$

In this case, if $\mathbf{V}=\mathbf{K}$ is a Killing vector field, then $\mathfrak{E}_{\mathbf{K}} \mathbf{g}=0$ and we have

$$
d \underset{\mathbf{g}}{\star} \mathscr{J}_{K}=\underset{\mathbf{g}}{\star}[(\mathbf{D} \cdot \mathbb{W})(\mathbf{K})]
$$

and the current 3-form field $\underset{\mathrm{g}}{\star} \mathscr{J}_{K}$ is closed; that is, $d \underset{\mathrm{g}}{\star} \mathscr{J}_{K}=$ 0 , or equivalently (taking into account the definition of the Hodge coderivative operator $\delta$ )

$$
{ }_{\mathbf{g}} \mathscr{J}_{K}=0
$$

In resume, when we have Killing vector fields ${ }^{8} \mathbf{K}_{i}, i=$ $1,2, \ldots, n$, "covariant conservation" of the tensor field $\mathbb{W}$, that is, $\mathbf{D} \cdot \mathbb{W}=0$, implies in genuine conservation laws for the currents $\mathscr{J}_{K_{i}}$ that from $\delta_{\mathrm{g}} \mathscr{F}_{K_{i}}=0$, we can using Stokes theorem build the scalar conserved quantities

$$
\mathscr{E}\left(K_{i}\right):=\frac{1}{8 \pi} \int_{\Sigma^{\prime}} \star_{\mathrm{g}} \mathscr{J}_{K_{i}},
$$

where $N$ is the region where $\mathscr{J}_{K}$ has support and $\partial N=\Sigma+$ $\Sigma^{\prime}+\boldsymbol{\Xi}$, where $\Sigma, \Sigma^{\prime}$ are spacelike surfaces and $\mathscr{J}_{K_{i}}$ is null at $\boldsymbol{\Xi}$ (spatial infinity).

\subsection{Conserved Currents in GRT Associated with Killing Vector} Fields. Before studying the conditions for the existence or not of genuine energy-momentum conservation laws in GRT, let us recall from Appendix C.3.4 that in Minkowski space-time $^{9}\left(M \simeq \mathbb{R}^{4}, \boldsymbol{\eta}, D, \tau_{\boldsymbol{\eta}}, \uparrow\right)$ we can introduce global coordinates $\left\{x^{\mu}\right\}$ (in Einstein-Lorentz-Poincaré gauge) such that $\boldsymbol{\eta}\left(e_{\mu}, e_{\nu}\right)=\eta_{\mu \nu}$ and $D_{e_{\mu}} e_{\nu}=0$, where $\left\{e_{\mu}=\partial / \partial x^{\mu}\right\}$ is simultaneously a global tetrad and a coordinate basis. Also $\left\{\vartheta^{\mu}=d x^{\mu}\right\}$ is a global cotetrad and a coordinate cobasis.

Moreover, $e_{\mu}=\partial / \partial x^{\mu}$ are also Killing vector fields in $\left(M \simeq \mathbb{R}^{4}, \boldsymbol{\eta}\right)$ and thus we have for a closed physical system (consisting of particles and fields in interaction living in Minkowski space-time and whose equations of motion are derived from a variational principle with a Lagrangian density invariant under space-time translations) that the currents $^{10}$

$$
\begin{array}{r}
\mathscr{T}_{\alpha}:=\mathscr{J}_{\partial / \partial x^{\alpha}}=T_{\alpha \nu} \vartheta^{\nu} \in \sec \bigwedge^{1} T^{*} M \hookrightarrow \mathscr{C} \ell(M, \eta), \\
M \simeq \mathbb{R}^{4}
\end{array}
$$

are the conserved energy-momentum 1-form fields of the physical system under consideration, for which we know that the quantity (recall (C.38))

$$
\mathbf{P}=\left.P_{\alpha} \vartheta^{\alpha}\right|_{o}=P_{\alpha} \mathbf{E}^{\alpha}
$$

with

$$
P_{\alpha}=\int_{\mathbf{g}}^{\star \mathscr{T}_{\alpha}}
$$

is the components of the conserved energy-momentum covector (CEMC) $\mathbf{P}$ of the system.

2.3.1. Limited Possibility to Construct a CEMC in GRT. Now, recall that in GRT a gravitational field generated by an energy-momentum $\mathbf{T}$ is modelled by a Lorentzian space$\operatorname{time}^{\mathrm{Il}}\left(M, \mathbf{g}, \mathbf{D}, \tau_{\mathbf{g}}, \uparrow\right)$ where the relation between $\mathbf{g}$ and $\mathbf{T}$ is given by Einstein equation which using the orthonormal bases $\left\{\mathbf{e}_{\alpha}\right\}$ and $\left\{\gamma^{\alpha}\right\}$ introduced above reads

$$
G_{\beta}^{\alpha}=R_{\beta}^{\alpha}-\frac{1}{2} \delta_{\beta}^{\alpha} R=-T_{\beta}^{\alpha} .
$$

Moreover, defining $\mathbf{G}=G_{\beta}^{\alpha} \gamma^{\beta} \otimes \mathbf{e}_{\alpha}$ and recalling that $\mathbf{T}=$ $T_{\beta}^{\alpha} \gamma^{\beta} \otimes \mathbf{e}_{\alpha}$, it is

$$
\begin{aligned}
& \mathbf{D} \cdot \mathbf{G}=0, \\
& \mathbf{D} \cdot \mathbf{T}=0 .
\end{aligned}
$$

Based only on the contents of Section 2.1, given that D • $\mathbf{T}=0$, it may seem at first sight ${ }^{12}$ that the only possibility to construct conserved energy-momentum currents $\mathscr{T}_{\alpha}$ in GRT is for models of the theory where appropriate Killing vector fields (such that one is timelike and the other three are spacelike) exist. However, an arbitrarily given Lorentzian manifold $(M, \mathbf{g}, D)$ in general does not have such Killing vector fields.

Remark 3. Moreover, even if it is the case that if in a particular model $(M, \mathbf{g}, D)$ of GRT there exist one timelike and three spacelike Killing vector fields, we can construct the scalar invariants quantities $P_{\alpha}, \alpha=0,1,2,3$, given by (27), we cannot define an energy-momentum covector $\mathbf{P}$ analogous to the one given by (26). This is so because in this case to have a conserved covector like $\mathbf{P}$ it is necessary to select a $\gamma^{\alpha}$ at a fixed point of the manifold. But in general there is no physically meaningful way to do that, except if $M$ is asymptotically flat $^{13}$ in which case we can choose a chart such that at spatial infinity $\lim _{|\vec{x}| \rightarrow \infty} \gamma^{\alpha}\left(x^{0}, \vec{x}\right)=\vartheta^{\alpha}=d x^{\alpha}$ and $\lim _{|\vec{x}| \rightarrow \gamma} \mathbf{g}_{\mu \nu}=\eta_{\mu \nu}$.

Thus, parodying Sachs and $\mathrm{Wu}$ [4] we must say that nonexistence of genuine conservation laws for energymomentum (and also angular momentum) in GRT is a shame.

Remark 4. Despite what has been said above and the results of Section 2.1 we next show that there exists trivially an infinity of conserved currents (the Komar currents) in any Lorentzian space-time modelling a gravitational field in GRT. We discuss the meaning and disclose the form of these currents, a result possible due to a notable decomposition of the square of the Dirac operator acting on sections of the Clifford bundle $\mathscr{C} \ell(M, \mathrm{~g})$. 
Remark 5. We end this subsection recalling that in order to produce genuine conservation laws in a field theory of gravitation with the gravitational field equations equivalent (in a precise sense) to Einstein equation it is necessary to formulate the theory in a parallelizable manifold and to dispense the Lorentzian space-time structure of GRT. Details of such a theory may be found in [5].

2.4. Komar Currents: Their Mathematical and Physical Meaning. Let $\mathbf{A} \in \sec T M$ be the generator of a one-parameter group of diffeomorphisms of $M$ in the space-time structure $\left\langle M, \mathbf{g}, \mathbf{D}, \tau_{\mathbf{g}}, \uparrow\right\rangle$ which is a model of a gravitational field generated by $\mathbf{T} \in \sec T_{1}^{1} M$ (the matter fields energymomentum tensor) in GRT. It is quite obvious that if we define $F=d A$, where $A=\mathbf{g}(\mathbf{A},) \in \sec \bigwedge^{1} T^{*} M \hookrightarrow \mathscr{C} \ell(M, \mathbf{g})$, then the current

$$
\mathbf{J}_{A}=\underset{\mathbf{g}}{\delta} F
$$

is conserved; that is,

$$
\underset{\mathbf{g}}{\delta \mathbf{J}_{A}}=0
$$

Surprisingly such a trivial mathematical result seems to be very important for people working in GRT who call $\mathbf{J}_{A}$ the Komar current ${ }^{14}[6]$. Komar called ${ }^{15}$

$$
\mathfrak{E}=\int_{V} \underset{\mathbf{g}}{\star \mathbf{J}_{A}}=\int_{\partial V} \stackrel{\star}{\mathbf{g}} F
$$

the generalized energy.

To understand why $\mathbf{J}_{A}$ is considered important in GRT write the action for the gravitational plus matter and nongravitational fields as

$$
\mathscr{A}=\int \mathscr{L}_{\mathrm{g}}+\int \mathscr{L}_{m}=-\frac{1}{2} \int R \tau_{\mathrm{g}}+\int \mathscr{L}_{m} .
$$

Now, the equations of motion for $\mathbf{g}$ can be obtained considering its variation under an (infinitesimal) diffeomorphism $h: M \rightarrow M$ generated by $\mathbf{A}$. We have that $\mathbf{g} \mapsto \mathbf{g}^{\prime}=$ $h^{*} \mathbf{g}=\mathbf{g}+\boldsymbol{\delta}^{0} \mathbf{g}$ where ${ }^{16}$ the variation $\boldsymbol{\delta}^{0} \mathbf{g}=-\mathfrak{E}_{\mathrm{A}} \mathbf{g}$. Taking into account Cartan's magical formula $\left(\mathfrak{E}_{\mathbf{A}} \mathbf{M}=A_{\mathbf{g}} d \mathbf{M}+d\left(\underset{\mathbf{g}}{A_{\lrcorner}} \mathbf{M}\right)\right.$, for any $\left.\mathbf{M} \in \sec \bigwedge T^{*} M\right)$, we have

$$
\begin{aligned}
\boldsymbol{\delta}^{0} \mathscr{A} & =\int \boldsymbol{\delta}^{0} \mathscr{L}_{g}+\int \boldsymbol{\delta}^{0} \mathscr{L}_{m}=-\int \mathfrak{E}_{\mathrm{A}} \mathscr{L}_{g}-\int \mathfrak{E}_{\mathrm{A}} \mathscr{L}_{m} \\
& \left.\left.=-\int d(A\lrcorner \mathscr{L}_{g}\right)-\int d(A\lrcorner \mathscr{L}_{m}\right):=\int d(\underset{\mathrm{g}}{\star} \mathscr{C}),
\end{aligned}
$$

where

$$
\left.\left.{ }_{\mathrm{g}}^{\star} \mathscr{C}=-A\right\lrcorner \mathscr{L}_{g}-A\right\lrcorner \mathscr{L}_{m}+K
$$

with $d K=0$.

To proceed introduce a coordinate chart with coordinates $\left\{x^{\mu}\right\}$ for the region of interest $U \subset M$. Recall that $\mathscr{G}^{\mu}=\mathscr{R}^{\mu}-$ $(1 / 2) R \vartheta^{\mu}$ are the Einstein 1 -form fields ${ }^{17}$, with $\mathscr{R}^{\mu}=R_{\nu}^{\mu} \boldsymbol{\vartheta}^{\mu}$ being the Ricci 1 -forms and $R$ the curvature scalar. Einstein equation obtained from the variation principle $\boldsymbol{\delta}^{0} \mathscr{A}=0$ is $\mathscr{E}^{\mu}:=\mathscr{G}^{\mu}+\mathscr{T}^{\mu}=0$, with $\mathscr{T}^{\mu}=T_{\nu}^{\mu} \boldsymbol{\vartheta}^{\nu}$ being the energymomentum 1-form fields and moreover $D_{\mu} G^{\mu \nu}=0=D_{\mu} T^{\mu \nu}$.

Next write explicitly the action as [7]

$$
\begin{aligned}
\mathscr{A}= & -\frac{1}{2} \int R \sqrt{-\operatorname{det} \mathbf{g}} d x^{0} d x^{1} d x^{2} d x^{3} \\
& +\int L_{m} \sqrt{-\operatorname{det} \mathbf{g}} d x^{0} d x^{1} d x^{2} d x^{3} .
\end{aligned}
$$

We have immediately

$$
\begin{aligned}
& \boldsymbol{\delta}^{0} \mathscr{A}=-\frac{1}{2} \int E^{\mu \nu}\left(\mathfrak{E}_{\mathbf{A}} \mathbf{g}\right)_{\mu \nu} \sqrt{-\operatorname{det} \mathbf{g}} d x^{0} d x^{1} d x^{2} d x^{3} \\
& =-\int E^{\mu \nu} D_{\mu} A_{\nu} \sqrt{-\operatorname{det} \mathbf{g}} d x^{0} d x^{1} d x^{2} d x^{3} \\
& =-\int D_{\mu}\left(E^{\mu \nu} A_{\nu}\right) \sqrt{-\operatorname{det} \mathbf{g}} d x^{0} d x^{1} d x^{2} d x^{3}
\end{aligned}
$$

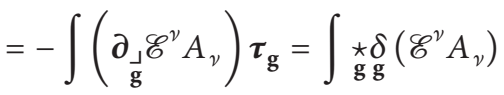

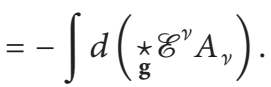

From (34) and (37) we have

$$
\int d\left(\underset{\mathbf{g}}{\star \mathscr{C}^{v}} A_{v}\right)+d(\underset{\mathbf{g}}{\star} \mathscr{C})=0
$$

and thus

$$
\underset{\mathbf{g}}{\delta}\left(\mathscr{E}^{v} A_{\nu}\right)+\underset{\mathbf{g}}{\delta} \mathscr{C}=0
$$

Thus, the current $\mathscr{C} \in \sec \bigwedge^{1} T^{*} M$ is conserved if the field equations $\mathscr{E}^{\nu}=0$ are satisfied. An equation (in component form) equivalent to (39) already appears in [6] (and also previously in [8]) that took $\mathscr{C}=\mathscr{E}^{\nu} K_{\nu}+N$ with $\underset{\mathbf{g}}{\delta} N=0$.

Here, to continue, we prefer to write an identity involving only $\boldsymbol{\delta}^{0} \mathscr{A}_{g}=\int \boldsymbol{\delta}^{0} \mathscr{L}_{g}$. Proceeding exactly as before we get putting $\mathscr{G}(A)=\mathscr{G}^{\mu} A_{\mu}$ that there exists $\mathbf{N} \in \sec \bigwedge^{1} T^{*} M$ such that

$$
\underset{\mathbf{g}}{\delta} \mathscr{G}(A)+\underset{\mathbf{g}}{\delta \mathbf{N}}=0
$$

and we see that we can identify

$$
\mathbf{N}:=-\mathscr{G}^{\mu} A_{\mu}+L,
$$

where $\underset{\mathbf{g}}{\delta} L=0$. Now, we claim the following.

Proposition 6. There exists $L \in \sec \bigwedge^{1} T^{*} M$ such that

$$
\mathbf{N}=-\mathscr{G}^{\mu} A_{\mu}+L=\underset{\mathbf{g}}{\delta} d A=-\mathbf{J}_{A}
$$

where $\mathbf{J}_{A}$ was defined in (30). 
Proof. To prove our claim we suppose from now on that $\bigwedge T^{*} M \hookrightarrow \mathscr{C} \ell(M, \mathrm{~g}){ }^{18}$.

Then it is possible to write

$$
\begin{aligned}
\mathscr{G}^{\mu} A_{\mu} & =\mathscr{R}^{\mu} A_{\mu}-\frac{1}{2} R A=\partial \wedge \partial A-\frac{1}{2} R A \\
& =\partial \wedge \partial A+\partial \cdot \partial A-\frac{1}{2} R A-\partial \cdot \partial A \\
& =\partial^{2} A-\frac{1}{2} R A-\partial \cdot \partial A \\
& =-\underset{\mathbf{g}}{\delta} d A-d \delta_{\mathbf{g}} A-\frac{1}{2} R A-\partial \cdot \partial A,
\end{aligned}
$$

where $\partial \wedge \partial$ is the Ricci operator and $\partial \cdot \partial=\square$ is the D'Alembertian operator. Then we take

$$
-\mathscr{G}^{\mu} A_{\mu}-d \delta_{\mathbf{g}} A-\frac{1}{2} R A-\partial \cdot \partial A=\underset{\mathbf{g}}{\delta} d A
$$

and of course it is ${ }^{19}$

$$
L=-d \delta \delta_{\mathbf{g}} A-\frac{1}{2} R A-\partial \cdot \partial A
$$

proving the proposition.

Now that we found a $L$ satisfying (42) we investigate if we can give some nontrivial physical meaning to such $\mathbf{N}=-\mathbf{J}_{A} \epsilon$ $\sec \bigwedge^{1} T^{*} M$.

2.4.1. Determination of the Explicit Form of $\mathbf{J}_{A}$. We recall that the extensor field $\mathrm{T}$ acts on $A$ as

$$
\mathbf{T}(A)=\mathscr{T}^{\mu} A_{\mu}
$$

Thus, since

$$
\mathscr{G}^{\mu} A_{\mu}=-\mathbf{T}(A)
$$

we have from (44)

$$
\underset{\mathbf{g}}{\delta} d A=\mathbf{T}(A)-d \delta_{\mathbf{g}} A-\frac{1}{2} R A-\partial \cdot \partial A .
$$

We can write (48) taking into account that $R=\operatorname{tr} \mathbf{T}=T_{\mu}^{\mu}$ and putting $F:=d A$ and that

$$
\delta F=-\mathbf{J}_{A}
$$

where [9]

$$
\mathbf{J}_{A}=-\mathbf{T}(A)+\frac{1}{2} \operatorname{tr} \mathbf{T} A+d \delta_{\mathbf{g}} A+\partial \cdot \partial A .
$$

Equation (50) gives the explicit form for the Komar current ${ }^{20}$. Moreover, taking into account that $\underset{\mathrm{g}}{\delta} F=\underset{\mathrm{g}}{\star} \underset{\mathrm{g}}{\star} F$, it is

$$
\begin{aligned}
d \star F & =\underset{\mathbf{g}}{\star^{-1}}\left(-\mathbf{T}(A)+\frac{1}{2} \operatorname{tr} \mathbf{T} A+\underset{\mathbf{g}}{d \delta_{0}} A+\partial \cdot \partial A\right) \\
& =\underset{\mathbf{g}}{\star}\left(-\mathbf{T}(A)+\frac{1}{2} \operatorname{tr} \mathbf{T} A+d \delta_{\mathbf{g}} A+\partial \cdot \partial A\right)
\end{aligned}
$$

and thus taking into account Stokes theorem

$$
\int_{\mathscr{V}} d \underset{\mathbf{g}}{\star} F=\int_{\partial \mathscr{V}} \stackrel{\star}{\mathbf{g}} F=\int_{\mathscr{V}} \stackrel{\star}{\mathbf{g}} \mathbf{J}_{A} .
$$

Moreover, since $\underset{\mathbf{g}}{d} \mathbf{J}_{A}=0$ we have that

$$
0=\int_{N} \underset{\mathbf{g}}{d \star \mathbf{J}_{A}}=\int_{\partial N} \stackrel{\star}{\mathbf{g}} \mathbf{J}_{A}
$$

and thus $\int_{\Sigma_{1}} \star \mathbf{J}_{A}\left(\partial N=\Sigma_{1}-\Sigma_{2}+\Xi\right)$ is a conserved quantity. We arrive at the conclusion that taking $\mathscr{V} \subset \Sigma_{1}\left(\partial \mathscr{V}=\mathcal{S}_{R}\right)$ as a ball of radius $R$ and making $R \rightarrow \infty$ the quantity

$$
\begin{aligned}
\mathscr{E} & \equiv \mathscr{E}(A):=\frac{1}{8 \pi} \int_{\mathcal{S}_{R}} \underset{\mathbf{g}}{\star F} \\
& =\frac{1}{8 \pi} \int_{V} \stackrel{\star}{\mathbf{g}}\left(\mathbf{T}(A)-\frac{1}{2} A \operatorname{tr} \mathbf{T}-d \delta_{\mathbf{g}} A-\partial \cdot \partial A\right)
\end{aligned}
$$

is conserved.

Remark 7. It is very important to realize that quantity $\mathscr{E}$ defined by (54) is a scalar invariant, that is, its value does not depend on the particular reference frame $\mathbf{Z}$ and (nacs $\mid \mathbf{Z}$ ) (the naturally adapted coordinate chart adapted to $\mathbf{Z})^{21}$. But, of course, for each particular vector field $\mathbf{A} \in \sec T M$ (which generates a one-parameter group of diffeomorphisms) we have a different $\mathscr{E}(A)$ and the different $\mathscr{E}(A)$ 's are not related as components of a covector.

Remark 8. As we already remarked an equation equivalent to (54) has already been obtained in [6] that called (as said above) that quantity the conserved generalized energy. But to the best of our knowledge (55) is new and appears for the first time in [9].

However, considering that for each $\mathbf{A} \in \sec T M$ that generates a one-parameter group of diffeomorphisms of $M$ we have a conserved quantity it is not in our opinion appropriate to think about this quantity as a generalized energy. Indeed, why should the energy depend on terms like $d \delta A$ and $\partial \cdot \partial A$ if $A$ is not a dynamical field?

We know that [10] when $\mathbf{A}=\mathbf{K}$ is a Killing vector field it is $\underset{\mathbf{g}}{\delta} A=0$ and $\boldsymbol{\partial} \cdot \boldsymbol{\partial} A=-\mathbf{T}(A)+(1 / 2) \operatorname{tr} \mathbf{T} A$ and thus (55) reads

$$
\mathscr{E}=\frac{1}{4 \pi} \int_{N} \star \mathrm{g}\left(\mathrm{T}(A)-\frac{1}{2} A \operatorname{tr} \mathbf{T}\right)
$$

which is a well known conserved quantity ${ }^{22}$. For a Schwarzschild space-time, as is well known, $\mathbf{A}=\partial / \partial t$ is a timelike Killing vector field and in this case since the components of $\mathbf{T}$ are $T_{v}^{\mu}=(8 \pi / \sqrt{-\operatorname{det} \mathbf{g}}) \rho(r) v^{\mu} v_{v}$ and $v^{i} v_{j}=0\left(\right.$ since $\left.v^{\mu}=\left(1 / \sqrt{g_{00}}\right)(1,0,0,0)\right)$ we get $\mathscr{E}=m$.

Remark 9. Note that the conserved quantity given by (56) differs in general from the conserved quantity obtained with the current defined in (9) when $V=K$ which holds in any structure $(M, \mathbf{g}, D)$ with the conditions given there. However, in the particular case analyzed above, (56) and (24) give the same result. 
Remark 10. Originally Komar obtained the same result as in (56) directly from (54) supposing that the generator of the one-parameter group of diffeomorphisms was $\mathbf{A}=\partial / \partial t$. So, he got $\mathscr{E}=m$ by pure chance. If he had picked another vector field generator of a one-parameter group of diffeomorphisms $\mathbf{A} \neq \partial / \partial t$, he, of course, would have not obtained that result.

Remark 11. The previous remark shows clearly that the construction of Komar currents does not solve the energymomentum conservation problem for a system consisting of the matter and nongravitational fields plus the gravitational field in GRT.

Indeed, to claim that a solution for a meaningful definition for the energy-momentum of the total system ${ }^{23}$ exist, it is necessary to find a way to define a total conserved energy-momentum covector for the total system as it is possible to do in field theories in Minkowski space-time (recall Section C.3.4). This can only be done if the space-time structure modelling a gravitational field (generated by the matter fields energy-momentum tensor T) possesses appropriate additional structure, or if we interpret the gravitational field as a field in the Faraday sense living in Minkowski spacetime. More details are in $[5,11]$.

2.4.2. The Maxwell-Like Equation $\partial F=\mathbf{J}_{A}$ Encodes Einstein Equation. From (49) where $F=d A(A=\mathbf{g}(\mathbf{A})$,$) with$ $\mathbf{A} \in \sec T M$ being an arbitrary generator of a one-parameter group of diffeomorphisms of $M$ (part of the structure $\left.\left(M, \mathbf{g}, D, \boldsymbol{\tau}_{\mathbf{g}}, \uparrow\right)\right)$ taking into account that $d F=0$, we get the Maxwell-like equation (MLE)

$$
\partial F=\mathbf{J}_{A}
$$

with a well defined conserved current. Of course, as we already said, there is an infinity of such equations. Each one encodes Einstein equation; that is, given the form of $\mathbf{J}_{A}$ (see (50)), we can get back (43), which gives immediately Einstein equation (EE). In this sense we can claim that

$$
\mathrm{EE} \mathbf{G}=\mathbf{T} \text { and the MLE } \boldsymbol{\partial F}=\mathbf{J}_{A} \text { are equivalent. }
$$

Remark 12. Finally it is worthy to emphasize that the above results show that in GRT there are infinity of conservation laws, one for each vector field generator of a one-parameter group of diffeomorphisms and, so, Noether's theorem in GRT which follows from the supposition that the Lagrangian density is invariant under the diffeomorphism group gives only identities, that is, an infinite set of conserved currents, each one encoding as we saw above Einstein equation.

It is now time to analyze the possible generalized conservation laws and their implications for the motions of probe single-pole particles in Lorentzian and teleparallel de Sitter space-time structures, where these structures are not supposed to represent models of gravitational fields in GRT, and compare these results with the ones in GRT. This will be one of the next sections.

\section{The Lorentzian de Sitter $M^{\mathrm{dSL}}$ Structure and Its Conformal Representation}

Let $\mathrm{SO}(1,4)$ and $\mathrm{SO}(1,3)$ be, respectively, the special pseudoorthogonal groups in $\mathbb{R}^{1,4}=\left\{\stackrel{\circ}{M}=\mathbb{R}^{5}, \stackrel{\circ}{\mathrm{g}}\right\}$ and in $\mathbb{R}^{1,3}=$ $\left\{\mathbb{R}^{4}, \boldsymbol{\eta}\right\}$, where $\stackrel{\mathrm{g}}{\mathrm{i}}$ is a metric of signature $(1,4)$ and $\boldsymbol{\eta}$ is a metric of signature $(1,3)$. The manifold $M=\mathrm{SO}(1,4) / \mathrm{SO}(1,3)$ will be called the de Sitter manifold. Since

$$
M=\operatorname{SO}(1,4) / \operatorname{SO}(1,3) \approx \mathbb{R} \times S^{3}
$$

this manifold can be viewed as a brane (a submanifold) in the structure $\mathbb{R}^{1,4}$. We now introduce a Lorentzian space-time, that is, the structure $M^{\mathrm{dSL}}=\left(M=\mathbb{R} \times S^{3}, \mathbf{g}, \mathbf{D}, \tau_{\mathbf{g}}, \uparrow\right)$ which will be called Lorentzian de Sitter space-time structure where if $\boldsymbol{\iota}: \mathbb{R} \times S^{3} \rightarrow \mathbb{R}^{5}, \mathbf{g}=\boldsymbol{\iota}^{*} \stackrel{\circ}{\mathbf{g}}$ and $\mathbf{D}$ is the parallel projection on $M$ of the pseudo-Euclidian metric compatible connection in $\mathbb{R}^{1,4}$ (details in [12]). As is well known, $M^{\mathrm{dSL}}$ is a spacetime of constant Riemannian curvature. It has ten Killing vector fields. The Killing vector fields are the generators of infinitesimal actions of the group $\mathrm{SO}(1,4)$ (called the de Sitter group) in $M=\mathbb{R} \times S^{3} \approx \operatorname{SO}(1,4) / \operatorname{SO}(1,3)$. The group $\mathrm{SO}(1,4)$ acts transitively ${ }^{24}$ in $\mathrm{SO}(1,4) / \mathrm{SO}(1,3)$, which is thus a homogeneous space (for $\mathrm{SO}(1,4)$ ).

We now recall the description of the manifold $\mathbb{R} \times S^{3}$ as a pseudosphere (a submanifold) of radius $\ell$ of the pseudoEuclidean space $\mathbb{R}^{1,4}=\left\{\mathbb{R}^{5}, \stackrel{\circ}{\mathrm{g}}\right\}$. If $\left(X^{0}, X^{1}, X^{2}, X^{3}, X^{4}\right)$ are the global coordinates of $\mathbb{R}^{1,4}$, then the equation representing the pseudosphere is

$$
\left(X^{0}\right)^{2}-\left(X^{1}\right)^{2}-\left(X^{2}\right)^{2}-\left(X^{3}\right)^{2}-\left(X^{4}\right)^{2}=-\ell^{2} .
$$

Introducing conformal coordinates ${ }^{25}\left\{x^{\mu}\right\}$ by projecting the points of $\mathbb{R} \times S^{3}$ from the "north pole" to a plane tangent to the "south pole" we see immediately that $\left\{x^{\mu}\right\}$ covers all $\mathbb{R} \times S^{3}$ except the "north pole." We immediately find that

$$
\mathbf{g}=\mathbf{i}^{*} \stackrel{\circ}{\mathbf{g}}=\Omega^{2} \eta_{\mu \nu} d x^{\mu} \otimes d x^{\nu},
$$

where

$$
\begin{aligned}
X^{\mu} & =\Omega x^{\mu}, \\
X^{4} & =-\ell \Omega\left(1+\frac{\sigma^{2}}{4 \ell^{2}}\right), \\
\Omega & =\left(1-\frac{\sigma^{2}}{4 \ell^{2}}\right)^{-1}, \\
\sigma^{2} & =\eta_{\mu \nu} x^{\mu} x^{\nu} .
\end{aligned}
$$

Since the north pole of the pseudosphere is not covered by the coordinate functions, we see that (omitting two dimensions) the region of the space-time as seen by an observer living in the south pole is the region inside the socalled absolute of Cayley-Klein of equation

$$
t^{2}-x^{2}=4 \ell^{2} \text {. }
$$

In Figure 1 we can see that all timelike curves (1) and (2) and lightlike (3) curve start in the "past horizon" and end on the "future horizon." 


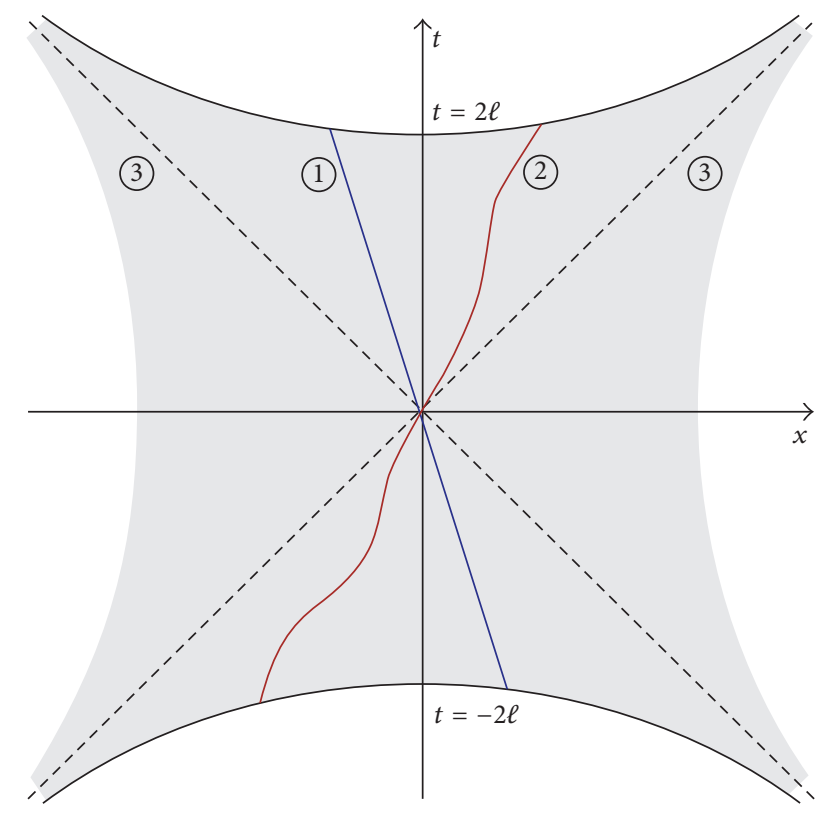

Figure 1: Conformal representation of de Sitter space-time. Note that the "observer" space-time is the interior of the Cayley-Klein absolute $t^{2}-\vec{x}^{2}=4 \ell^{2}$.

\section{On the Geodesics of $M^{\mathrm{dSL}}$}

In a classic book by Hawking and Ellis [13] we can read on page 126 the following statement:

de Sitter spacetime is geodesically complete; however there are points in the space which cannot be joined to each other by any geodesic.

Unfortunately many people do not realize that the points that cannot be joined by a geodesic are some points which can be joined by a spacelike curve (living in region 3 in Figure 1). So, these curves are never the path of any particle. A complete and thoughtful discussion of this issue is given in an old and excellent article by Schmidt [14].

Remark 13. Having said that, let us recall that among the Killing vector fields ${ }^{26}$ of $\left(M=\mathbb{R} \times S^{3}, \mathbf{g}\right)$ there are one timelike and three spacelike vector fields (which are called the translation Killing vector fields in physical literature). So, many people have thought for a long time that this permits the formulation of an energy-momentum conservation law in de Sitter space-time $M^{\mathrm{dSL}}$ structure. However, the fact is that what can really be done is the obtainment of conserved quantities like $P_{\alpha}$ in (27). But we cannot obtain in $M^{\mathrm{dSL}}$ structure an energy-momentum covector like $\mathbf{P}$ for a given closed physical system using an equation similar to (26). This is because de Sitter space-time is not asymptotically flat and so there is no way to physically determine a point to fix the $\gamma^{\alpha}$ to use in an equation similar to (26).

Now, the "translation" Killing vector fields of de Sitter space-time are expressed in the coordinate basis $\left\{\partial_{\mu}=\partial / \partial x^{\mu}\right\}$ (where $\left\{x^{\mu}\right\}$ are the conformal coordinates introduced in the last section) by $^{27}$ :

$$
\Pi_{\alpha}=\xi_{\alpha}^{\mu} \partial_{\mu}
$$

with (putting $\ell=1$, for simplicity)

$$
\begin{aligned}
& \xi_{\alpha}^{\mu}=\delta_{\alpha}^{\mu}-\left(\frac{\eta_{\alpha \nu} x^{\nu} x^{\mu}}{2}-\frac{\sigma^{2}}{4} \delta_{\alpha}^{\mu}\right), \\
& \xi_{\alpha}^{0}=\delta_{\alpha}^{0}-\left(\frac{\sigma^{2}}{4} \frac{\eta_{\alpha \nu} x^{\nu} x^{0}}{2}-\frac{\sigma^{2}}{4} \delta_{\alpha}^{0}\right), \\
& \xi_{\alpha}^{1}=\delta_{\alpha}^{1}-\left(\frac{\sigma^{2}}{4} \frac{\eta_{\alpha \nu} x^{\nu} x^{1}}{2}-\frac{\sigma^{2}}{4} \delta_{\alpha}^{1}\right), \\
& \xi_{\alpha}^{2}=\delta_{\alpha}^{2}-\left(\frac{\sigma^{2}}{4} \frac{\eta_{\alpha \nu} x^{\nu} x^{2}}{2}-\frac{\sigma^{2}}{4} \delta_{\alpha}^{2}\right), \\
& \xi_{\alpha}^{3}=\delta_{\alpha}^{3}-\left(\frac{\sigma^{2}}{4} \frac{\eta_{\alpha \nu} x^{\nu} x^{3}}{2}-\frac{\sigma^{2}}{4} \delta_{\alpha}^{3}\right) .
\end{aligned}
$$

So, we want now to investigate whether there is some region of de Sitter space-time where the "translational" Killing vector fields are linearly independent.

In order to proceed, we take an arbitrary vector field $\mathbf{V}=$ $V^{\mu} \partial_{\mu} \in \sec T \mathscr{U}$. If $\Pi_{\alpha}$ are linearly independent in a region $\mathcal{U}^{\prime} \subset \mathcal{U} \subset \mathbb{R} \times S^{3}$, then we can write

$$
\mathbf{V}=V^{\mu} \partial_{\mu}=\mathbf{V}^{\alpha} \Pi_{\alpha}=\mathbf{V}^{\alpha} \xi_{\alpha}^{\mu} \partial_{\mu}
$$

So, the condition for the existence of a nontrivial solution for the $\mathbf{V}^{\alpha}$ is that

$$
\operatorname{det}\left(\begin{array}{cccc}
\xi_{0}^{0} & \xi_{0}^{1} & \xi_{0}^{2} & \xi_{0}^{3} \\
\xi_{1}^{0} & \xi_{1}^{1} & \xi_{1}^{2} & \xi_{1}^{3} \\
\xi_{2}^{0} & \xi_{2}^{1} & \xi_{2}^{2} & \xi_{2}^{3} \\
\xi_{3}^{0} & \xi_{3}^{1} & \xi_{3}^{2} & \xi_{3}^{3}
\end{array}\right) \neq 0
$$

Thus, putting

$$
\chi_{\mu}:=\left(\frac{\sigma^{2}}{4} \frac{\left(x^{\mu}\right)^{2}}{2}-\frac{\sigma^{2}}{4}\right)
$$

we need to evaluate the determinant of the matrix

$$
\left(\begin{array}{cccc}
1-\chi_{0} & \left(\frac{\sigma^{2}}{4} \frac{t x}{2}\right) & \left(\frac{\sigma^{2}}{4} \frac{t y}{2}\right) & \left(\frac{\sigma^{2}}{4} \frac{t z}{2}\right) \\
-\left(\frac{\sigma^{2}}{4} \frac{x t}{2}\right) & 1-\chi_{1} & -\left(\frac{\sigma^{2}}{4} \frac{x y}{2}\right) & -\left(\frac{\sigma^{2}}{4} \frac{x z}{2}\right) \\
-\left(\frac{\sigma^{2}}{4} \frac{y t}{2}\right) & -\left(\frac{\sigma^{2}}{4} \frac{y x}{2}\right) & 1-\chi_{2} & -\left(\frac{y z}{\left(m^{2} / 4\right) 2}\right) \\
-\left(\frac{\sigma^{2}}{4} \frac{z t}{2}\right) & -\left(\frac{\sigma^{2}}{4} \frac{z x}{2}\right) & -\left(\frac{\sigma^{2}}{4} \frac{z y}{2}\right) & 1-\chi_{3}
\end{array}\right)
$$


Then

$$
\begin{aligned}
\operatorname{det} & {\left[\xi_{\alpha}^{\mu}\right] } \\
= & -\frac{1}{512} \sigma^{10}-\frac{3}{32} \sigma^{6}+\frac{1}{16} \sigma^{6}+\sigma^{2}+1-\frac{3}{128} \sigma^{8} \\
& -\frac{1}{8} \sigma^{4} \\
& +\frac{1}{1024} \sigma^{8}\left(3 t^{2} x^{2} y^{2} z^{2}+t^{2} y^{2} z^{2}-x^{2} y^{2} z^{2}+y^{2} z^{2}\right) \\
& +\frac{1}{512} \sigma^{8}\left(t^{2} x^{2} y^{2}+t^{2} x^{2} z^{2}\right) \\
& +\frac{1}{128} \sigma^{6} t^{2} x^{2}\left(y^{2}+z^{2}\right) \\
& +\frac{1}{256} \sigma^{6}\left(t^{2} y^{2} z^{2}-x^{2} y^{2} z^{2}+2 y^{2} z^{2}\right) \\
& +\frac{1}{64} \sigma^{4}\left(t^{2} x^{2} y^{2} z^{2}+y^{2} z^{2}-x^{2} y^{2} z^{2}\right) \\
& +\frac{1}{16} \sigma^{2}\left(t^{2} y^{2} z^{2}-x^{2} y^{2} z^{2}-2 y^{2} z^{2}\right)+\frac{3}{8} \sigma^{4} \\
& -\frac{1}{4} y^{2} z^{2}+\frac{1}{256} \sigma^{8} .
\end{aligned}
$$

In order to analyze this expression we put (without loss of generality; see the reason in [14]) $y=z=0$. In this case

$$
\operatorname{det}\left[\xi_{\alpha}^{\mu}\right]=\frac{1}{512}\left(\sigma^{2}+4\right)^{3}\left(-\sigma^{4}+2 \sigma^{2}+8\right)
$$

which is null on the Caley-Klein absolute, that is, at the points

$$
t^{2}-\vec{x}^{2}=4,
$$

and also on the spacelike hyperbolas given by

$$
\begin{aligned}
& t^{2}-\vec{x}^{2}=-2, \\
& t^{2}-\vec{x}^{2}=-4 .
\end{aligned}
$$

So, we have proved that the translational Killing vector fields are linearly independent in all the subregions inside the Cayley-Klein absolute except in the points of the hyperbolas $t^{2}-\vec{x}^{2}=-2$ and $t^{2}-\vec{x}^{2}=-4$.

This result is very important for the following reason. Timelike geodesics in de Sitter space-time structure $M^{\mathrm{dSL}}$ are (as is well known) the curves $\sigma: s \rightarrow \sigma(s)$, where $s$ is proper time along $\sigma$, which minimizes or maximizes the length function [15], that is, calling $\sigma_{*}=\mathbf{u}$ the velocity of a "particle" of mass $m$ following a timelike geodesic; we have that the equation of the geodesic is obtained by finding an extreme of the action, written here in sloop notation, as

$$
I[\sigma]=-m \int_{\sigma} d s=-m \int_{\sigma}\left(g_{\mu \nu} d x^{\mu} d x^{\nu}\right)^{1 / 2} .
$$

As is well known, the determination of an extreme for $I[\sigma]$ is given by evaluating the first variation ${ }^{28}$ of $I[\sigma]$, that is,

$$
\boldsymbol{\delta}^{0} I[\sigma]=m \int \mathfrak{E}_{\mathbf{Y}}\left(g_{\mu \nu} d x^{\mu} d x^{\nu}\right)^{1 / 2},
$$

and putting $\boldsymbol{\delta}^{0} I[\sigma]=0$. The result, as is well known, is the geodesic equation

$$
\mathbf{D}_{\mathbf{u}} \mathbf{u}=0
$$

Now, taking into account that the Killing vector fields determine a basis inside the Cayley-Klein absolute we write

$$
\mathbf{u}=u^{\mu} \partial_{\mu}=U^{\alpha} \Pi_{\alpha}=U^{\alpha} \xi_{\alpha}^{\mu} \partial_{\mu} .
$$

We now define the "hybrid" connection coefficients ${ }^{29} \Gamma_{\cdot \mu \alpha}^{\beta \cdot .}$ by

$$
\begin{aligned}
& \mathbf{D}_{\partial_{\mu}} \Pi_{\alpha}:=\boldsymbol{\Gamma}_{\cdot \mu \alpha}^{\beta \cdot \cdot} \Pi_{\beta}, \\
& \mathbf{D}_{\partial_{\mu}} \Pi^{\alpha}:=-\Gamma_{\cdot \mu \beta}^{\alpha \cdot \cdot} \Pi^{\beta}
\end{aligned}
$$

and write the geodesic equation as

$$
\begin{aligned}
\mathbf{D}_{\mathbf{u}} \mathbf{u} & =u^{\mu} \mathbf{D}_{\partial \mu}\left(U^{\alpha} \boldsymbol{\Pi}_{\alpha}\right) \\
& =\left(u^{\mu} \partial_{\mu} U^{\alpha}\right) \Pi_{\alpha}+u^{\mu} U^{\alpha} \mathbf{D}_{\partial \mu} \Pi_{\alpha} \\
& =\left(u^{\mu} \partial_{\mu} U^{\beta}\right) \Pi_{\beta}+u^{\mu} U^{\alpha} \Gamma_{\cdot \mu \alpha}^{\beta \cdot \cdot} \boldsymbol{\Pi}_{\beta} \\
& =\left(\frac{d U^{\beta}}{d s}+u^{\mu} U^{\alpha} \Gamma_{\cdot \mu \alpha}^{\beta \cdot \cdot}\right) \Pi_{\beta}=0
\end{aligned}
$$

or

$$
\frac{d U^{\beta}}{d s}+u^{\mu} U^{\alpha} \Gamma_{\cdot \mu \alpha}^{\beta \cdot *}=0
$$

which on multiplying by the "mass" $m$ and calling $\pi^{\beta}=m U^{\beta}$ and $\pi_{\rho}=g_{\rho \beta} \pi^{\beta}$ can be written equivalently as

$$
\frac{d \pi_{\rho}}{d s}-\Gamma_{\cdot \mu \rho}^{\beta \cdot \cdot} u^{\mu} \pi_{\beta}=0,
$$

which looks like Equation (37) in [1].

We want now to investigate the following question: is (82) the same as Equation (37) in [1]?

To know the answer to the above question recalls that in [1] authors investigate the variation of $I[\sigma]$ under a variation of the curves $\sigma(s) \mapsto \sigma(s, \ell)$ induced by a coordinate transformation $x^{\mu} \mapsto x^{\prime \mu}+\delta_{\Pi} x^{\mu}$, where they put

$$
\boldsymbol{\delta}_{\Pi} x^{\mu}=\xi_{\rho}^{\mu}(x) \boldsymbol{\delta} x^{\rho}
$$

with $\xi_{\rho}^{\mu}(x)$ being the components of the Killing vector fields $\Pi_{\alpha}$ (recall (65)) and where it is said that $\delta x^{\rho}$ is an ordinary variation. However in [1] we cannot find what authors mean by ordinary variation, and so $\delta x^{\rho}$ is not defined.

So, to continue our analysis we recall that if $\delta x^{\rho}=\varepsilon^{\alpha}$ are constants (but arbitrary), then $\delta_{\Pi} x^{\mu}$ corresponds to a diffeomorphism generated by a Killing vector field $\Pi=\varepsilon^{\alpha} \Pi_{\alpha}$. However, if $\delta x^{\rho}=\lambda^{\rho}(x)$ are infinitesimal arbitrary functions (i.e., $\left|\lambda^{\rho}(x)\right| \ll 1$ ), then the notation $\delta_{\Pi} x^{\mu}$ is misleading since $\xi_{\rho}^{\mu} \lambda^{\rho}(x)$ is the $\boldsymbol{\delta}^{0} I[\sigma]$ variation generated by a quite arbitrary vector field $\mathbf{Y}=\lambda^{\rho}(x) \xi_{\rho}^{\mu}(x) \partial_{\mu}=\lambda^{\rho}(x) \Pi_{\rho}=Y^{\mu} \partial_{\mu}$. In this case we get from $\boldsymbol{\delta}^{0} I[\sigma]=0$ the geodesic equation. 
4.1. Curves Obtained from Constrained Variations. So, let us study the constrained variation when $\delta x^{\rho}=\varepsilon^{\alpha}$ are constants (but arbitrary). We denote the constrained variation by $\boldsymbol{\delta}^{c} I[\sigma]$. In this case starting from (75)

$$
\boldsymbol{\delta}^{c} I[\sigma]=-m \int_{\sigma}\left\{u^{\gamma}\left(\mathbf{D}_{\gamma} u_{\beta}\right) \xi_{\rho}^{\beta}\right\} \delta x^{\rho} d s,
$$

where (taking account of notations already introduced)

$$
\begin{aligned}
u^{\gamma} \mathbf{D}_{e_{\gamma}}\left(u_{\beta} e^{\beta}\right) & =u^{\gamma}\left(\mathbf{D}_{\gamma} u_{\beta}\right) e^{\beta}, \\
\mathbf{D}_{\gamma} u_{\beta} & =\partial_{\gamma} u_{\beta}-\Gamma_{\cdot \gamma \beta}^{\tau \cdot \cdot} u_{\tau}, \\
\mathbf{D}_{e_{\gamma}} e_{\beta} & =\Gamma_{\cdot \gamma \beta}^{\tau \cdot \cdot} e_{\tau}, \\
\mathbf{D}_{e_{\gamma}} e^{\beta} & =-\Gamma_{\cdot \gamma \tau}^{\beta \cdot \cdot} e^{\tau} .
\end{aligned}
$$

We can write

$$
\begin{aligned}
\boldsymbol{\delta}^{c} I[\sigma]= & -m \int\left\{u^{\gamma}\left(\mathbf{D}_{\gamma} u_{\beta}\right) \xi_{\rho}^{\beta}\right\} \boldsymbol{\delta} x^{\rho} d s \\
= & -m \int\left\{u^{\gamma}\left\{\left(\mathbf{D}_{\gamma}\left(u^{\beta} \xi_{\rho \beta}\right)\right)\right\} \boldsymbol{\delta} x^{\rho} d s\right. \\
& +\frac{m}{2} \int\left\{u^{\gamma} u^{\beta}\left(\mathbf{D}_{\gamma} \xi_{\beta \rho}+\mathbf{D}_{\beta} \xi_{\rho}^{\gamma}\right\} \boldsymbol{\delta} x^{\rho} d s\right. \\
= & -m \int\left\{u^{\gamma}\left(\mathbf{D}_{\gamma}\left(\mathbf{u} \cdot \boldsymbol{\Pi}_{\rho}\right)\right)\right\} \boldsymbol{\delta} x^{\rho} d s \\
= & -m \int\left[u^{\gamma} \mathbf{D}_{\gamma}\left(\mathcal{U}_{\rho}\right)\right\} \boldsymbol{\delta} x^{\rho} d s
\end{aligned}
$$

and $\boldsymbol{\delta}^{\mathcal{c}} I[\sigma]=0$ implies

$$
u^{\gamma} \mathbf{D}_{\gamma}\left(m U_{\rho}\right)=0 \text {. }
$$

Equation (87) with $\pi_{\rho}=m U_{\rho}$ can be written as

$$
\frac{d}{d s} \pi_{\rho}-u^{\gamma} \pi_{\beta} \Gamma_{\cdot \cdot \rho}^{\beta \cdot *}=0
$$

which is Equation (37) in [1]. Note that this equations looks like the geodesic equation written as (82) above, but it is in fact different since of course, recalling (79), it is $\Gamma_{\cdot \gamma \rho}^{\beta \cdot *} \neq \Gamma_{\cdot \gamma \rho}^{\beta \cdot}$. In [1] $\pi_{\rho}=m U_{\rho}$ is unfortunately wrongly interpreted as the components of a covector field over $\sigma$ which is supposed to be the energy-momentum covector of the particle, because authors of [1] were supposed to prove that this equation could be derived from Papapetrou's method, which is not the case as we show in Section 6.

\section{Generalized Energy-Momentum Conservation Laws in de Sitter Space-Time Structures}

5.1. Lie Algebra of the de Sitter Group. Given a structure $(\stackrel{M}{2}$ $\mathbb{R}^{5}, \stackrel{\circ}{\mathbf{g}}$, ) introduced in Section 3 define $\mathbf{J}_{A B} \in \sec T \stackrel{\circ}{M}$ by

$$
\mathbf{J}_{A B}:=\eta_{A C} X^{C} \frac{\partial}{\partial X^{B}}-\eta_{B C} X^{C} \frac{\partial}{\partial X^{A}} .
$$

These objects are generators of the Lie algebra so $(1,4)$ of the de Sitter group.

Using the bases $\left\{\partial_{\mu}\right\},\left\{d x^{\mu}\right\}$ introduced above the ten Killing vector fields of de Sitter space-time are the fields $\mathbf{J}_{\alpha 4} \in$ $\sec T M$ and $\mathbf{J}_{\mu \nu} \in \sec T M$ and it is ${ }^{30}$

$$
\begin{aligned}
\mathbf{J}_{\alpha 4} & =\eta_{\alpha C} X^{C} \frac{\partial}{\partial X^{4}}-\eta_{4 C} X^{C} \frac{\partial}{\partial X^{\alpha}}=\ell \mathbf{P}_{\alpha}-\frac{1}{4 \ell} \mathbf{K}_{4 \alpha} \\
& =\ell \partial_{\alpha}-\frac{1}{4 \ell}\left(2 \eta_{\alpha \lambda} x^{\lambda} x^{\nu}-\sigma^{2} \delta_{\alpha}^{\nu}\right) \partial_{\nu}, \\
\mathbf{J}_{\mu \nu} & =\eta_{\mu \kappa} X^{\kappa} \frac{\partial}{\partial X^{\nu}}-\eta_{\nu \kappa} X^{\kappa} \frac{\partial}{\partial X^{\mu}} \\
& =\eta_{\mu \lambda} x^{\lambda} \mathbf{P}_{\nu}-\eta_{\nu \lambda} x^{\lambda} \mathbf{P}_{\mu} .
\end{aligned}
$$

$\mathbf{J}_{\alpha 4} \in \sec T M$ and $\mathbf{J}_{\mu \nu} \in \sec T M$ satisfy the Lie algebra $\operatorname{so}(1,4)$ of the de Sitter group, this time acting as a transformation group acting transitively in de Sitter space-time. We have

$$
\begin{aligned}
& {\left[\mathbf{J}_{\alpha 4}, \mathbf{J}_{\beta 4}\right]=\mathbf{J}_{\alpha \beta},} \\
& {\left[\mathbf{J}_{\alpha \beta}, \mathbf{J}_{\lambda 4}\right]=\eta_{\lambda \beta} \mathbf{J}_{\alpha 4}-\eta_{\lambda \alpha} \mathbf{J}_{\beta 4},} \\
& {\left[\mathbf{J}_{\alpha \beta}, \mathbf{J}_{\lambda \tau}\right]=\eta_{\alpha \lambda} \mathbf{J}_{\beta \tau}+\eta_{\beta \tau} \mathbf{J}_{\alpha \lambda}-\eta_{\beta \lambda} \mathbf{J}_{\alpha \tau}-\eta_{\alpha \tau} \mathbf{J}_{\beta \lambda} .}
\end{aligned}
$$

It is usual in physical applications to define

$$
\Pi_{\alpha}=\frac{\mathbf{J}_{\alpha 4}}{\ell}
$$

for then we have

$$
\begin{aligned}
& {\left[\boldsymbol{\Pi}_{\alpha}, \boldsymbol{\Pi}_{\beta}\right]=\frac{1}{\ell^{2}} \mathbf{J}_{\alpha \beta},} \\
& {\left[\mathbf{J}_{\alpha \beta}, \boldsymbol{\Pi}_{\lambda}\right]=\eta_{\lambda \beta} \boldsymbol{\Pi}_{\alpha}-\eta_{\lambda \alpha} \boldsymbol{\Pi}_{\beta},} \\
& {\left[\mathbf{J}_{\alpha \beta}, \mathbf{J}_{\lambda \tau}\right]=\eta_{\alpha \lambda} \mathbf{J}_{\beta \tau}+\eta_{\beta \tau} \mathbf{J}_{\alpha \lambda}-\eta_{\beta \lambda} \mathbf{J}_{\alpha \tau}-\eta_{\alpha \tau} \mathbf{J}_{\beta \lambda} .}
\end{aligned}
$$

The Killing vector fields $\mathbf{J}_{\alpha \beta}$ satisfy the Lie algebra $s o(1,3)$ (of the special Lorentz group).

Remark 14. From (94) we see that when $\ell \mapsto \infty$ the Lie algebra of $s o(1,4)$ goes into the Lie algebra of the Poincaré group $\mathfrak{P}$ which is the semidirect sum of the group of translations in $\mathbb{R}^{4}$ plus component of the special Lorentz group; that is, $\mathfrak{P}=\left(\mathbb{R}^{4} \boxplus \operatorname{SO}(1,3)\right)$. This is eventually the justification for physicists to call $\Pi_{\alpha}$ the "translation" generators of the de Sitter group.

However, it is necessary to have in mind that whereas the translation subgroup $\mathbb{R}^{4}$ of $\mathfrak{P}$ acts transitively in Minkowski space-time manifold, the set $\left\{\boldsymbol{\Pi}_{\alpha}\right\}$ does not close in a subalgebra of the de Sitter algebra and thus it is impossible in general to find $\exp \left(\lambda^{\alpha} \Pi_{\alpha}\right)$ such that given arbitrary $x, y \in \mathbb{R} \times S^{3}$ it is $y=\exp \left(\lambda^{\alpha} \boldsymbol{\Pi}_{\alpha}\right) x$. Only the whole group $\mathrm{SO}(1,4)$ acts transitively on $\mathbb{R} \times S^{3}$. 
Casimir Invariants. Now, if $\left\{\mathbf{E}^{A}=d X^{A}\right\}$ is an orthonormal basis for the structure $\mathbb{R}^{1,4}=\left(\mathbb{R}^{5}, \stackrel{\circ}{\mathbf{g}}\right)$ define the angular momentum operator as the Clifford algebra valued operator

$$
\mathbf{J}=\frac{1}{2} \mathbf{E}^{A} \wedge \mathbf{E}^{B} \mathbf{J}_{A B}
$$

Taking into account the results of Appendix A its (Clifford) square is

$$
\left.\mathbf{J}^{2}=\mathbf{J}\right\lrcorner \mathbf{J}+\mathbf{J} \wedge \mathbf{J}=-\mathbf{J} \cdot \mathbf{J}+\mathbf{J} \wedge \mathbf{J}
$$

It is immediate to verify that $\mathbf{J}^{2}$ is invariant under the transformations of the de Sitter group. $\mathbf{J}\lrcorner \mathbf{J}$ is (a constant apart) the first invariant Casimir operator of the de Sitter group. The second invariant Casimir operator of the de Sitter is related to $\mathbf{J} \wedge \mathbf{J}$. Indeed, defining

$$
\left.\mathbf{W}:=\underset{\mathrm{g}}{\star} \frac{1}{8 \ell}(\mathbf{J} \wedge \mathbf{J})=\frac{1}{8 \ell}(\mathbf{J} \wedge \mathbf{J})\right\lrcorner \tau_{\mathrm{g}}^{\circ},
$$

one can easily show (details in [16]) that

$$
\begin{aligned}
\mathbf{W} \cdot \mathbf{W} & \left.=\mathbf{W} \mathbf{W}=\mathbf{W}^{2}=-\frac{1}{64 \ell^{2}}(\mathbf{J} \wedge \mathbf{J})\right\lrcorner(\mathbf{J} \wedge \mathbf{J}) \\
& =-\frac{1}{64 \ell^{2}}(\mathbf{J} \wedge \mathbf{J}) \cdot(\mathbf{J} \wedge \mathbf{J}) \\
& =-\frac{1}{64 \ell^{2}}(\mathbf{J} \wedge \mathbf{J})(\mathbf{J} \wedge \mathbf{J})
\end{aligned}
$$

is indeed an invariant operator.

As is well known, the representations of the de Sitter group are classified by their Casimir invariants $I_{1}$ and $I_{2}$ which here following [17] we take as

$$
\begin{aligned}
I_{1} & =-\mathbf{J}_{\lrcorner} \mathbf{J}=\frac{1}{2 \ell^{2}} \mathbf{J}_{A B} \mathbf{J}^{A B} \\
& =\eta^{\alpha \beta} \Pi_{\alpha} \Pi_{\beta}+\frac{1}{2 \ell^{2}} \eta^{\alpha \lambda} \eta^{\beta \tau} \mathbf{J}_{\alpha \beta} \mathbf{J}_{\lambda \tau}=M^{2}, \\
I_{2} & =\mathbf{W}^{2}=\mathbb{W}^{A} \mathbb{W}_{A}=\eta^{\alpha \beta} \mathbf{V}_{\alpha} \mathbf{V}_{\beta}+\frac{1}{\ell^{2}}\left(\mathbb{W}_{4}\right)^{2},
\end{aligned}
$$

where $M \in \mathbb{R}$ and the fields $\mathbb{W}_{A}$ and $\mathbf{V}_{\alpha}$ are defined by

$$
\begin{aligned}
\mathbb{W}_{A} & :=\frac{1}{8 \ell} \varepsilon_{A B C D E} \mathbf{J}^{A B} \mathbf{J}^{D E} \\
\mathbf{V}_{\alpha} & :=-\frac{1}{2} \varepsilon_{4 \alpha \lambda \mu \nu} \eta^{\lambda \rho} \boldsymbol{\Pi}_{\rho} \mathbf{J}^{\mu \nu},
\end{aligned}
$$

from where it follows that

$$
\mathbb{W}_{4}=\frac{1}{8} \varepsilon_{4 \mu \nu \rho \tau} \mathbf{J}^{\mu \nu} \mathbf{J}^{\mu \nu}
$$

In the limit when $\ell \mapsto \infty$ we get the Casimir operators of the special Lorentz group

$$
\begin{aligned}
& I_{1} \longmapsto \mathbf{P}_{\alpha} \mathbf{P}^{\alpha}=m^{2}, \\
& I_{2} \longmapsto \eta^{\alpha \beta} \mathbf{V}_{\alpha} \mathbf{V}_{\beta}=m^{2} s(s+1),
\end{aligned}
$$

where $m \in \mathbb{R}$ and $s=0,1 / 2,1,3 / 2, \ldots$
We see that $\Pi_{\alpha}$ looks like the components of an energymomentum vector $\mathbf{P}=\left.P_{\alpha} \vartheta^{\alpha}\right|_{o}$ of a closed physical system (see (26)) in the Minkowski space-time of Special Relativity, for which $\mathbf{P}^{2}=m^{2}$, with $m$ being the mass of the system. However, take into account that whereas $P_{\alpha}$ are simple real numbers, $\Pi_{\alpha}$ are vector fields.

Moreover, take into account that $\eta^{\alpha \beta} \Pi_{\alpha} \Pi_{\beta}$ is not an invariant; that is, it does not commute with the generators of the Lie algebra of the de Sitter group.

5.2. Generalized Energy-Momentum Covector for a Closed System in the Teleparallel de Sitter Space-Time Structure. In this section we suppose that $\left(M=\mathbb{R} \times S^{3}, \mathbf{g}, \tau_{\mathbf{g}}, \uparrow\right)$ is the physical arena where Physics take place.

We know that $\left(M=\mathbb{R} \times S^{3}, \mathbf{g}\right)$ has ten Killing vector fields and four of them (one timelike and three spacelike, $\Pi_{\alpha}$, $\alpha=0,1,2,3)$ generated "translations." Thus if we suppose that $\left(M=\mathbb{R} \times S^{3}, \mathbf{g}, \tau_{\mathbf{g}}, \uparrow\right)$ is populated by interacting matter fields $\left\{\phi_{1}, \ldots, \phi_{n}\right\}$ with dynamics described by a Lagrangian formalism we can construct as described in Appendix C.1 the conserved currents

$$
\mathscr{J}_{\boldsymbol{\Pi}_{\alpha}}=\mathscr{J}_{\alpha}^{\beta} \boldsymbol{\gamma}_{\beta}=\mathscr{J}_{\alpha}^{\mu} \boldsymbol{\vartheta}_{\mu}
$$

where taking into account that $\boldsymbol{\delta}_{\Pi_{\alpha}}^{0}=-\mathfrak{E}_{\Pi_{\alpha}}$ and denoting by $\boldsymbol{\delta}_{\Pi_{\alpha}}$ each particular local variation generated by $\Pi_{\alpha}$ (recall (64)) we have

$$
\boldsymbol{\delta}_{\Pi_{\alpha}} \phi_{A}=\delta_{\Pi_{\alpha}}^{0} \phi_{A}+\delta x^{\nu} \partial_{\nu} \phi_{A}
$$

and thus we write

$$
\mathscr{J}_{\alpha}^{\mu}=\pi_{A}^{\mu} \delta_{\Pi_{\alpha}} \phi_{A}+\Upsilon_{\nu}^{\mu} \delta_{\Pi_{\alpha}}^{0} x^{\mu}=\Lambda_{\nu}^{\mu} \xi_{\alpha}^{v}+\Upsilon_{\nu}^{\mu} \xi_{\alpha}^{\nu},
$$

where

$$
\Lambda_{\nu}^{\mu} \xi_{\alpha}^{\nu}:=\pi_{A}^{\mu} \boldsymbol{\delta}_{\Pi_{\alpha}} \phi_{A}
$$

In (103) $\left\{\gamma^{\beta}\right\}$ is the dual basis of the orthonormal basis $\left\{\mathbf{e}_{\alpha}\right\}$ defined by ${ }^{31}$

$$
\mathbf{e}_{\alpha}:=\frac{\Pi_{\alpha}}{\mathbf{g}\left(\Pi_{\alpha}, \Pi_{\alpha}\right)} .
$$

From the conserved currents $\mathscr{J}_{\Pi_{\alpha}}$ we can obtain four conserved quantities $\left(P_{\alpha}\right.$ in (27)).

Remark 15. It is crucial to observe that the above results have been deduced without introduction of any connection in the structure $\left(M=\mathbb{R} \times S^{3}, \mathbf{g}, \tau_{\mathbf{g}}, \uparrow\right)$. However that structure is not enough for using the $P_{\alpha}$ to build a (generalized) covector analogous to the energy-momentum covector $\mathbf{P}$ (see (26)) of special relativistic theories.

If we add $\mathbf{D}$, the Levi-Civita connection of $\mathbf{g}$, to $(M=\mathbb{R} \times$ $\left.S^{3}, \mathbf{g}, \tau_{\mathbf{g}}, \uparrow\right)$ we get the Lorentzian de Sitter space-time structure $M^{\mathrm{dSP}}$ and defining a generalized energy-momentum tensor

$$
\Theta=\mathscr{J}_{\Pi_{\alpha}} \otimes e^{\alpha} \in \sec T_{1}^{1} M
$$


we know that $\delta_{\mathbf{g}} \mathscr{J}_{\boldsymbol{\Pi}_{\alpha}}=0$ implies $\mathbf{D} \cdot \boldsymbol{\Theta}=0$, a covariant "conservation" law.

However, the introduction of $M^{\mathrm{dSP}}$ in our game is of no help to construct a covector like $\mathbf{P}$ since in $M^{\mathrm{dSP}}$ vectors at different space-time points cannot be directly compared.

So, the question arises: is it possible to define a structure where $\forall x, y \in \mathbb{R} \times S^{3}$ we can define objects $P_{\alpha}^{\mathrm{dS}}$ such that

$$
\mathbf{P}_{\mathrm{dS}}=\left.P_{\alpha}^{\mathrm{dS}} \gamma^{\alpha}\right|_{x}=\left.P_{\alpha}^{\mathrm{dS}} \gamma^{\alpha}\right|_{y}
$$

defines a legitimate covector for a closed physical system living in a de Sitter structure $\left(M=\mathbb{R} \times S^{3}, \mathbf{g}, \tau_{\mathbf{g}}, \uparrow\right)$ and for which $\mathbf{P}_{\mathrm{dS}}$ can be said to be a kind of generalization of the momentum of the closed system in Minkowski space-time?

We show now that the answer is positive. We recall that $\mathbf{D}$ has been introduced in our developments only as a useful mathematical device and is quite irrelevant in the construction of legitimate conservation laws since the conserved currents $\mathscr{J}_{\Pi_{\alpha}}$ have been obtained without the use of any connection. So, we now introduce for our goal a teleparallel de Sitter space-time, that is, the structure $M^{\mathrm{dSTP}}=\left(M=\mathbb{R} \times S^{3}, \nabla, \tau_{\mathrm{g}}, \uparrow\right)$, where $\nabla$ is a metric compatible teleparallel connection defined by

$$
\nabla_{\mathbf{e}_{\alpha}} \mathbf{e}_{\beta}=\omega_{\cdot \alpha \beta}^{\kappa \cdot *} \mathbf{e}_{\kappa}=0
$$

Under this condition we know that we can identify all tangent and all cotangent spaces. So, we have, $\forall x, y \in \mathbb{R} \times S^{3}$,

$$
\begin{aligned}
& \left.\left.\mathbf{e}_{\alpha}\right|_{x} \simeq \mathbf{e}_{\alpha}\right|_{y}, \\
& \left.\left.\gamma^{\alpha}\right|_{x} \simeq \gamma^{\alpha}\right|_{y}=\mathbf{E}^{\alpha},
\end{aligned}
$$

where $\left\{\mathbf{E}^{\alpha}\right\}$ is a basis of a vector space $\mathscr{V} \simeq \mathbb{R}^{4}$.

Thus, in the structure $M^{\mathrm{dSTP}}$ (109) defines indeed a legitimate covector in $\mathscr{V} \simeq \mathbb{R}^{4}$ and thus permits a legitimate generalization of the concepts of energy-momentum covector obtained for physical theories in Minkowski space-time. The term generalization is a good one here because in the limit, where $\ell \rightarrow \infty, \Pi_{\alpha} \mapsto \partial / \partial x^{\alpha}, \Lambda_{\alpha}^{\kappa}=0$ and thus $\Theta_{\alpha}^{\kappa}=\Upsilon_{\alpha}^{\kappa}$.

5.3. The Conserved Currents $\mathbf{J}_{\Pi_{\alpha}}=\mathbf{g}\left(\boldsymbol{\Pi}_{\alpha},\right)$. To proceed we show that the translational Killing vector fields of the de Sitter structure $(M, \mathbf{g})$ determine trivially conserved currents

$$
\mathbf{J}_{\Pi_{\alpha}}=\mathbf{g}\left(\Pi_{\alpha},\right) \text {. }
$$

Indeed using the $M^{\mathrm{dSTP}}$ structure as a convenient device we recall the result proved in [10] that for each vector Killing $\mathbf{K}$ the one form field $K=\mathbf{g}(\mathbf{K}$,$) is such that \delta_{\mathbf{g}} K=0$. So, it is

$$
\delta \mathbf{J}_{\Pi_{\alpha}}=0
$$

and, of course, also

$$
\underset{\mathrm{g}}{\delta \mathbf{J}_{\Pi}}=0
$$

where

$$
\begin{aligned}
\mathbf{J}_{\Pi} & :=\mathbf{g}(\boldsymbol{\Pi},), \\
\Pi & :=\varepsilon^{\alpha} \Pi_{\alpha}
\end{aligned}
$$

with $\varepsilon^{\alpha}$ being real constants such that $\left|\varepsilon^{\alpha}\right| \ll 1$. Recalling from (91) that in projective conformal coordinate bases $\left(\left\{e_{\mu}=\partial_{\mu}\right\}\right.$ and $\left.\left\{\boldsymbol{\vartheta}^{\mu}=d x^{\mu}\right\}\right)$ the components of $\boldsymbol{\Pi}_{\alpha}$ are

$$
\xi_{\alpha}^{\mu}=\delta_{\alpha}^{\mu}-\frac{1}{4 \ell^{2}}\left(2 \eta_{\alpha \rho} x^{\rho} x^{\mu}-\sigma^{2} \delta_{\alpha}^{\mu}\right)
$$

we get that the conserved current $\mathbf{J}_{\Pi}$ is

$$
\mathbf{J}_{\Pi}=\varepsilon^{\alpha} \mathbf{J}_{\Pi_{\alpha}}=\varepsilon^{\alpha} \xi_{\alpha}^{\mu} \boldsymbol{\vartheta}_{\mu} .
$$

Remark 16. Take notice that, of course, this current is not the conserved current that we found in the previous section.

Remark 17. Take notice also that in [1] authors trying to generalize the results that follow from the canonical formalism for the case of field theories in Minkowski space-time suppose that they can eliminate the term $\pi_{A}^{\mu} \delta_{\Pi_{\alpha}} \phi_{A}$ from (105) by decree postulating a new kind of "local variation," call it $\boldsymbol{\delta}^{\prime}$, for which $\boldsymbol{\delta}^{\prime} \phi_{A}=0$. The fact is that such a "new kind of local variation" never appears in the canonical Lagrangian formalism; only $\delta \phi_{A}$ appears and in general it is not zero.

Now, the generalized canonical de Sitter energymomentum tensor is

$$
\boldsymbol{\Theta}=\mathscr{J}_{\boldsymbol{\Pi}_{\alpha}} \otimes e^{\alpha}=\Theta_{\alpha}^{\kappa} \boldsymbol{\vartheta}_{\kappa} \otimes e^{\alpha} \in \sec \bigwedge^{1} T^{*} M \otimes T M
$$

and making analogy with the case of Minkowski spacetime where $Y_{\alpha}^{\kappa}$ have been defined as the components of the canonical energy-momentum tensor ${ }^{32} \Upsilon$, we write $\Theta_{\alpha}^{\kappa}$, as

$$
\Theta_{\alpha}^{\kappa}=\Upsilon_{\alpha}^{\kappa}-\frac{1}{4 \ell^{2}} K_{\alpha}^{\kappa}+\Lambda_{\alpha}^{\kappa}
$$

Thus each one of the genuine conservation laws $d \underset{\mathrm{g}}{\star} \mathscr{J}_{\boldsymbol{\Pi}_{\alpha}}=0$, $\alpha=0,1,2,3$ reads in coordinate basis components as

$$
\begin{aligned}
& \left(\partial_{\mu} \Upsilon_{\alpha}^{\kappa}-\Gamma_{\cdot \lambda \nu}^{\lambda \cdot *} \Upsilon_{\alpha}^{\kappa}\right)-\frac{1}{4 \ell^{2}}\left(\partial_{\mu} K_{\alpha}^{\mu}-\Gamma_{\cdot \lambda \nu}^{\lambda \cdot \cdot} K_{\alpha}^{\kappa}\right) \\
& -\left(\partial_{\mu} \Lambda_{\alpha}^{\kappa}-\Gamma_{\cdot \lambda \nu}^{\lambda \cdot \cdot} \Lambda_{\alpha}^{\kappa}\right)=\frac{1}{\sqrt{-\operatorname{det} \mathbf{g}}} \partial_{\mu}\left(\sqrt{-\operatorname{det} \mathbf{g}} \Upsilon_{\alpha}^{\mu}\right) \\
& -\frac{1}{4 \ell^{2}} \frac{1}{\sqrt{-\operatorname{det} \mathbf{g}}} \partial_{\mu}\left(\sqrt{-\operatorname{det} \mathbf{g} K_{\alpha}^{\mu}}\right) \\
& +\frac{1}{\sqrt{-\operatorname{det} \mathbf{g}}} \partial_{\mu}\left(\sqrt{-\operatorname{det} \mathbf{g} \Lambda_{\alpha}^{\mu}}\right)=0
\end{aligned}
$$

or

$$
\begin{aligned}
\partial_{\mu}\left[\sqrt{-\operatorname{det} \mathbf{g}}\left(\Upsilon_{\alpha}^{\mu}-\frac{1}{4 \ell^{2}} K_{\alpha}^{\mu}+\Lambda_{\alpha}^{\mu}\right)\right] & =0 \Longleftrightarrow \\
\mathbf{D}_{\mu} \Theta_{\alpha}^{\mu} & =0 .
\end{aligned}
$$


Remark 18. Now, recalling that the relation of the connection coefficients of the bases $\left\{\mathbf{e}_{\alpha}\right\}$ of the Levi-Civita connection D of $\mathbf{g}$ (denoted $\Gamma_{\cdot \alpha \beta}^{\kappa^{*} \cdot}$ ) and the coefficients of the basis $\left\{\mathbf{e}_{\alpha}\right\}$ of the teleparallel connection $\nabla$ of $\mathbf{g}\left(\right.$ denoted $\left.\overline{\boldsymbol{\Gamma}}_{\cdot \alpha \beta}^{\kappa \cdot *}\right)$ are [11]

$$
\overline{\boldsymbol{\Gamma}}_{\cdot \alpha \beta}^{\kappa \cdot \cdot}=\Gamma_{\cdot \alpha \beta}^{\kappa \cdot \cdot}+\Delta_{\cdot \alpha \beta}^{\kappa \cdot},
$$

where

$$
\Delta_{\cdot \alpha \beta}^{\kappa \cdot}:=-\frac{1}{2}\left(T_{\alpha \cdot \beta}^{\cdot \kappa \cdot}+T_{\beta \cdot \alpha}^{\cdot \kappa \cdot}-T_{\cdot \alpha \beta}^{\kappa \cdot \cdot}\right)
$$

are the components of the contorsion tensor and $T_{\cdot \alpha \beta}^{\kappa \cdot *}$ are the components of the torsion tensor of the connection $\nabla$, we can write $\mathbf{D} \bullet \boldsymbol{\Theta}=0$ (taking into account that $\overline{\boldsymbol{\Gamma}}_{\cdot \alpha \beta}^{\kappa \cdot \cdot}=0$ ) in components relative to orthonormal basis as

$$
\begin{aligned}
\mathbf{D}_{\alpha} \Theta_{\beta}^{\alpha} & :=\left(\mathbf{D}_{\mathbf{e}_{\alpha}} \Theta\right)_{\beta}^{\alpha}=\mathbf{e}_{\alpha}\left(\Theta_{\beta}^{\alpha}\right)+\Delta_{\alpha l}^{\alpha} \Theta_{\beta}^{l}-\Delta_{\alpha \beta}^{l} \Theta_{l}^{\alpha} \\
& =0 .
\end{aligned}
$$

On the other hand since $\nabla_{\alpha} \Theta_{\beta}^{\alpha}:=\left(\nabla_{\mathbf{e}_{\alpha}} \Theta\right)_{\beta}^{\alpha}=\mathbf{e}_{\alpha}\left(\Theta_{\beta}^{\alpha}\right)$ we have

$$
\nabla_{\alpha} \Theta_{\beta}^{\alpha}=-\Delta_{\alpha l}^{\alpha} \Theta_{\beta}^{l}+\Delta_{\alpha \beta}^{\iota} \Theta_{l}^{\alpha},
$$

which means that although $(\nabla \cdot \Theta)_{\beta}^{\alpha}:=\nabla_{\alpha} \Theta_{\beta}^{\alpha} \neq 0$ we can generate the conserved currents $\mathbf{J}_{\Pi_{\alpha}}$ in the teleparallel de Sitter space-time structure if (125) is satisfied.

Remark 19. To end this section and for completeness of the article it is necessary to mention that in a remarkable paper [18] the gravitational energy-momentum tensor in teleparallel gravity is discussed in detail. Also related papers are $[19,20]$. A complete list of references can be found in [21].

\section{Equation of Motion for a Single-Pole Mass in a GRT Lorentzian Space-Time}

In a classical paper Papapetrou derived the equations of motion of single-pole and spinning particles in GRT. Here we recall his derivation for the case of a single-pole-mass. We start recalling that in GRT the matter fields are described by an energy-momentum tensor that satisfies the covariant conservation law $\mathbf{D} \cdot \mathbf{T}=0$. If we introduce the relative tensor

$$
\mathfrak{T}=\mathbf{T} \otimes \boldsymbol{\tau}_{\mathbf{g}} \in \sec T_{1}^{1} M \otimes \bigwedge^{4} T^{*} M
$$

we have, recalling Appendix $\mathrm{E}$ and that $\mathbf{D} \cdot \mathbf{T}=0$, that

$$
\mathbf{D}_{\nu} \mathfrak{T}^{\mu \nu}+\Gamma_{\cdot \nu \alpha}^{\mu \cdot \cdot} \mathfrak{T}^{\alpha \nu}=0
$$

From (127) we have

$$
\partial_{\nu}\left(x^{\alpha} \mathfrak{T}^{\mu \nu}\right)=\mathfrak{T}^{\mu \alpha}-x^{\alpha} \Gamma_{\cdot v \alpha}^{\mu \cdot \cdot} \mathfrak{T}^{\alpha \nu}
$$

To continue we suppose that a single-pole mass (considered as a probe particle) is modelled by the restriction of the energy-momentum tensor $\mathbf{T}$ inside a "narrow" tube in the Lorentzian space-time representing a given gravitational field. Let us call $\mathbf{T}_{0}$ the restriction and notice that $\mathbf{D} \cdot \mathbf{T}_{0}=$ 0 . Inside the tube a timelike line $\gamma$ is chosen to represent the particle motion. We restrict our analysis to hyperbolic Lorentzian space-times for which a foliation $\mathbb{R} \times \mathcal{S}(\mathcal{S}$ is a 3 dimensional manifold) exists. We choose a parametrization for $\gamma$ such that its coordinates are $\mathbf{x}^{\mu}(\gamma(t))=X^{\mu}(t)$, where $t=x^{0}$. The probe particle is characterized by taking the coordinates of any point in the world tube to satisfy

$$
\delta x^{\mu}=x^{\mu}-X^{\mu} \ll 1 .
$$

According to Papapetrou a single-pole particle is one for which the integral ${ }^{33}$

$$
\int \mathfrak{T}_{o}^{\mu v} d v \neq 0
$$

and all other integrals

$$
\begin{gathered}
\int \delta x^{\alpha} \mathfrak{T}_{o}^{\mu v} d v \\
\int \delta x^{\alpha} \delta x^{\beta} \mathfrak{T}_{o}^{\mu \nu} d v
\end{gathered}
$$

are null. We now evaluate

$$
\begin{aligned}
\frac{d}{d t} \int \mathfrak{T}_{o}^{\mu 0} & =-\int \Gamma_{\cdot v \alpha}^{\mu \cdot} \mathfrak{T}_{o}^{\alpha \nu} d v, \\
\frac{d}{d t} \int x^{\alpha} \mathfrak{T}_{o}^{\mu 0} & =\int \mathfrak{T}_{o}^{\mu \alpha} d v-\int x^{\alpha} \Gamma_{\cdot v \alpha}^{\mu \cdot \cdot} \mathfrak{T}_{o}^{\alpha \nu} d v .
\end{aligned}
$$

Inside the world tube modelling the particle we can expand the connection coefficients as

$$
\Gamma_{\cdot v \alpha}^{\mu \cdot \cdot}={ }_{o} \Gamma_{\cdot v \alpha}^{\mu \cdot \cdot}+{ }_{o} \Gamma_{\cdot v \alpha, \kappa}^{\mu \cdot \cdot} \delta x^{\kappa}
$$

with ${ }_{o} \Gamma_{\cdot v \alpha}^{\mu \cdot \cdot}$ being the components of the connection in worldline $\gamma$. Then according to the definition of a single-pole particle we get from (132) along $\gamma$

$$
\begin{aligned}
\frac{d}{d t} \int \mathfrak{T}_{o}^{\mu 0}+{ }_{o} \Gamma_{\cdot \nu \alpha}^{\mu \cdot \cdot} \int \mathfrak{T}_{o}^{\alpha \nu} & =0, \\
\int \mathfrak{T}_{o}^{\mu \alpha} & =\frac{d X^{\mu}}{d t} \int \mathfrak{I}_{o}^{\nu 0} d v .
\end{aligned}
$$

Now, put

$$
\gamma_{*}=\mathbf{u}:=\frac{d X^{\mu}}{d s} e_{\mu},
$$

where $d s$ is proper time along $\gamma$, and define

$$
M^{\mu \alpha}=u^{0} \int \mathfrak{T}_{o}^{\mu \alpha} d v
$$

Equations (134) and (135) become

$$
\begin{aligned}
\frac{d}{d s}\left(\frac{M^{\mu 0}}{u^{0}}\right)+{ }_{o} \Gamma_{\cdot v \alpha}^{\mu \cdot} M^{\alpha \nu} & =0 \\
M^{\mu \alpha} & =u^{\mu} \frac{M^{\alpha 0}}{u^{0}} .
\end{aligned}
$$


So, $M^{\mu 0}=u^{\mu}\left(M^{00} / u^{0}\right)$ from where it follows that putting

$$
m:=\frac{M^{00}}{\left(u^{0}\right)^{2}}
$$

it is

$$
M^{\mu \alpha}=u^{\mu} \frac{M^{\alpha 0}}{u^{0}}=m u^{\mu} u^{\alpha}
$$

and we get

$$
\frac{d}{d s}\left(m u^{\mu}\right)+{ }_{o} \Gamma_{\cdot v \alpha}^{\mu \cdot \cdot} m u^{\nu} u^{\alpha}=0
$$

Now, the acceleration of the probe particle is $\mathbf{a}:=\mathbf{D}_{\mathbf{u}} \mathbf{u}$ and thus $\mathbf{g}(\mathbf{a}, \mathbf{u})=0$; that is,

$$
u_{\mu} \frac{d}{d s} u^{\mu}+{ }_{o} \Gamma_{\cdot \nu \alpha}^{\mu \cdot \cdot} m u^{\nu} u^{\alpha} u_{\mu}=0
$$

Multiplying (142) by $u_{\mu}$ and using (143) give

$$
\frac{d}{d s} m=0
$$

and then (142) says that $\gamma$ is a geodesic of the Lorentzian space-time structure; that is,

$$
\mathbf{D}_{\gamma_{*}} \gamma_{*}=0
$$

or

$$
\frac{d u^{\mu}}{d s}+{ }_{o} \Gamma_{\cdot v \alpha}^{\mu \cdot \cdot} m u^{\nu} u^{\alpha}=0 .
$$

\section{Equation of Motion for a Single-Pole Mass in a de Sitter Lorentzian Space-Time}

In this section we suppose that the arena where physical events take place is the de Sitter space-time structure $M^{\mathrm{dSL}}$ where fields live and interact, without never changing the metric g, which as we emphasize does not represent any gravitational field here; that is, we do not suppose here that $M^{\mathrm{dSL}}$ is a model of a gravitational field in GRT. As we learned in Section 4 since de Sitter space-time has one timelike and three spacelike Killing vector fields $\Pi_{\alpha}$ we can construct the conserved currents $\mathscr{J}_{\Pi_{\alpha}}=\Theta_{\alpha}^{\mu} \boldsymbol{\vartheta}_{\mu}$ (see (103)) from where we get $\mathbf{D} \bullet \boldsymbol{\Theta}=0$ with $\boldsymbol{\Theta}=\mathscr{J}_{\boldsymbol{\Pi}_{\alpha}} \otimes e^{\alpha}=\Theta_{\alpha}^{\kappa} \boldsymbol{\vartheta}_{\kappa} \otimes e^{\alpha}$.

Now, if we suppose that a probe-free single-pole particle (i.e., one for which its interaction with the remaining fields can be despised) is described by a covariant conserved tensor $\Theta$ in a narrow tube like the one introduced in the previous section, we can derive (using analog notations for $\gamma$, etc.) an equation like (134); that is,

$$
\begin{aligned}
& \frac{d}{d t} \int \Theta_{o}^{\mu 0} \sqrt{-\operatorname{det} \mathbf{g}_{o}} d v+{ }_{o} \Gamma_{\cdot v \alpha}^{\mu \cdot \cdot} \int \Theta_{o}^{\alpha \nu} \sqrt{-\operatorname{det} \mathbf{g}_{o}} d v \\
& \quad=0 .
\end{aligned}
$$

Now, we obtain an equation analogous to (138) with $M^{\alpha \nu}$ substituted by $N^{\alpha \nu}$; that is,

$$
\frac{d}{d s}\left(\frac{N^{\mu 0}}{u^{0}}\right)+{ }_{o} \Gamma_{\cdot \nu \alpha}^{\mu \cdot \cdot} N^{\alpha \nu}=0
$$

with

$$
N^{\alpha v}:=u^{0} \int \Theta_{o}^{\mu \alpha} \sqrt{-\operatorname{det} \mathbf{g}_{o}} d v
$$

Putting this time

$$
m:=\frac{N^{00}}{\left(u^{0}\right)^{2}}
$$

we get $N^{\mu \alpha}=u^{\mu}\left(N^{\alpha 0} / u^{0}\right)=m u^{\mu} u^{\alpha}$ and

$$
\frac{d}{d s}\left(m u^{\mu}\right)+{ }_{o} \Gamma_{\cdot v \alpha}^{\mu \cdot \cdot} m u^{\nu} u^{\alpha}=0
$$

from where we get exactly as in the previous section that $m=$ const and $\mathbf{D}_{\gamma_{*}} \gamma_{*}=0$.

Conclusion 1. Papapetrou method applied to the $M^{\mathrm{dSL}}$ structure gives for the motion of a free single-pole particle the geodesic equation $\mathbf{D}_{\gamma_{*}} \gamma_{*}=0$. Moreover, (151) is, of course, different from (88) contrary to conclusions of authors of [1]. It is also to be noted here that in [1] authors inferred correctly that Papapetrou method leads to an equation that looks like (147) for a single-pole particle moving in the $M^{\mathrm{dSL}}$ structure. The equation that looks like (147) in [1] is Equation (37) there, but where in place of $\Theta_{o}^{\alpha \nu}$ they used $\Upsilon_{o}^{\alpha \nu}-\left(1 / 4 \ell^{2}\right) \Upsilon_{o}^{\alpha \nu}$, because they believe it is possible to use a local variation of the fields that results in $\Lambda_{v}^{\mu}=0$.

\section{Appendix}

\section{A. Clifford Bundle Formalism}

Let $\left(M, \mathbf{g}, D, \tau_{\mathbf{g}}, \uparrow\right)$ be an arbitrary Lorentzian or RiemannCartan space-time structure. The quadruple $\left(M, \mathbf{g}, \tau_{\mathbf{g}}, \uparrow\right)$ denotes a four-dimensional time-oriented and spaceoriented Lorentzian manifold [4, 11]. This means that $\mathbf{g} \in \sec T_{2}^{0} M$ is a Lorentzian metric of signature $(1,3)$, $\tau_{\mathrm{g}} \in \sec \bigwedge^{4} T^{*} M$, and $\uparrow$ is a time-orientation (see details, e.g., in [4]). Here, $T^{*} M[T M]$ is the cotangent [tangent] bundle. $T^{*} M=\bigcup_{x \in M} T_{x}^{*} M, T M=\bigcup_{x \in M} T_{x} M$, and $T_{x} M \simeq T_{x}^{*} M \simeq \mathbb{R}^{1,3}$, where $\mathbb{R}^{1,3}$ is the Minkowski vector space $^{34}$. $D$ is a metric compatible connection; that is, $D \mathbf{g}=0$. When $D=\mathbf{D}$ is the Levi-Civita connection of $\mathbf{g}, \mathbf{R}^{\mathbf{D}} \neq 0$, and $\Theta^{\mathbf{D}}=0$, with $\mathbf{R}^{\mathbf{D}}$ and $\Theta^{\mathbf{D}}$ being, respectively, the curvature and torsion tensors of the connection. $D=\nabla$ is a Riemann-Cartan connection, $\mathbf{R}^{\nabla} \neq 0$, and $\Theta^{\nabla} \neq 0{ }^{35}$. Let $\mathrm{g} \in \sec T_{0}^{2} M$ be the metric of the cotangent bundle. The Clifford bundle of differential forms $\mathscr{C l}(M, \mathrm{~g})$ is the bundle of algebras, that is, $\mathscr{C} \ell(M, \mathrm{~g})=\bigcup_{x \in M} \mathscr{C} \ell\left(T_{x}^{*} M, \mathrm{~g}\right)$, where, $\forall x \in M, \mathscr{C} \ell\left(T_{x}^{*} M, \mathrm{~g}\right)=\mathbb{R}_{1,3}$, the so-called space-time algebra [11]. Recall also that $\mathscr{C l}(M, \mathrm{~g})$ is 
a vector bundle associated with the orthonormal frame bundle; that is, we have ${ }^{36} \mathscr{C l}(M, \mathrm{~g})=P_{\mathrm{SO}_{(1,3)}^{e}}(M) \times_{\mathrm{Ad}^{\prime}} \mathbb{R}_{1,3}$ $[22,23]$. Also, when $(M, \mathbf{g})$ is a spin manifold we can show that $^{37} \mathscr{C} \ell(M, \mathrm{~g})=P_{\operatorname{Spin}_{(1,3)}^{e}}(M) \times_{\mathrm{Ad}} \mathbb{R}_{1,3}$. For any $x \in M$, $\mathscr{C} \ell\left(T_{x}^{*} M,\left.\mathrm{~g}\right|_{x}\right)$ as a linear space over the real field $\mathbb{R}$ is isomorphic to the Cartan algebra $\wedge T_{x}^{*} M$ of the cotangent space. We have that $\bigwedge_{x}^{*} M=\bigoplus_{k=0}^{4} \bigwedge^{k} T_{x}^{*} M$, where $\bigwedge^{k} T_{x}^{*} M$ is the $\left(\begin{array}{l}4 \\ k\end{array}\right)$-dimensional space of $k$-forms. Then, sections of $\mathscr{C} \ell(M, \mathrm{~g})$ can be represented as a sum of nonhomogeneous differential forms, which will be called Clifford (multiform) fields. In the Clifford bundle formalism, of course, arbitrary basis can be used, but in this short review of the main ideas of the Clifford calculus we use mainly orthonormal basis. Let then $\left\{\mathbf{e}_{\alpha}\right\}$ be an orthonormal basis for $T U \subset T M$; that is, $\mathrm{g}\left(\mathbf{e}_{\alpha}, \mathbf{e}_{\beta}\right)=\eta_{\alpha \beta}=\operatorname{diag}(1,-1,-1,-1)$. Let $\gamma^{\alpha} \in$ $\sec \bigwedge^{1} T^{*} M \hookrightarrow \sec \mathscr{C} \ell(M, g)(\alpha=0,1,2,3)$ be such that the set $\left\{\boldsymbol{\gamma}^{\alpha}\right\}$ is the dual basis of $\left\{\mathbf{e}_{\alpha}\right\}$. Also, $\left\{\boldsymbol{\gamma}_{\alpha}\right\}$ is the reciprocal basis of $\left\{\gamma^{\alpha}\right\}$, that is, $\mathrm{g}\left(\boldsymbol{\gamma}^{\alpha}, \boldsymbol{\gamma}_{\beta}\right)=\delta_{\beta}^{\alpha}$, and $\left\{\mathbf{e}^{\alpha}\right\}$ is the reciprocal basis of $\left\{\mathbf{e}_{\alpha}\right\}$; that is, $\mathbf{g}\left(\mathbf{e}^{\alpha}, \mathbf{e}_{\beta}\right)=\delta_{\beta}^{\alpha}$.

A.1. Clifford Product. The fundamental Clifford product (in what follows to be denoted by juxtaposition of symbols) is generated by

$$
\gamma^{\alpha} \gamma^{\beta}+\gamma^{\beta} \gamma^{\alpha}=2 \eta^{\alpha \beta}
$$

and if $\mathscr{C} \in \sec \mathscr{C} \ell(M, g)$ we have

$$
\mathscr{C}=s+v_{\alpha} \gamma^{\alpha}+\frac{1}{2 !} f_{\alpha \beta} \gamma^{\alpha} \gamma^{\beta}+\frac{1}{3 !} t_{\alpha \beta \gamma} \gamma^{\alpha} \gamma^{\beta} \gamma^{\gamma}+p \gamma^{5}
$$

where $\tau_{\mathrm{g}}=\gamma^{5}=\gamma^{0} \gamma^{1} \gamma^{2} \gamma^{3}$ is the volume element and $s, v_{\alpha}$, $f_{\beta}, t_{\alpha \beta \gamma}, p \in \sec \bigwedge^{0} T^{*} M \hookrightarrow \sec \mathscr{C} \ell(M, \mathrm{~g})$.

For $A_{r} \in \sec \bigwedge^{r} T^{*} M \hookrightarrow \sec \mathscr{C} \ell(M, g)$ and $B_{s} \in$ $\sec \bigwedge^{s} T^{*} M \hookrightarrow \sec \mathscr{C} \ell(M, \mathrm{~g})$ we define the exterior product in $\mathscr{C} \ell(M, \mathrm{~g})(\forall r, s=0,1,2,3)$ by

$$
A_{r} \wedge B_{s}=\left\langle A_{r} B_{s}\right\rangle_{r+s}
$$

where \langle\rangle$_{k}$ is the component in $\bigwedge^{k} T^{*} M$ of the Clifford field. Of course, $A_{r} \wedge B_{s}=(-1)^{r s} B_{s} \wedge A_{r}$, and the exterior product is extended by linearity to all sections of $\mathscr{C} \ell(M, \mathrm{~g})$.

Let $A_{r} \in \sec \bigwedge^{r} T^{*} M \hookrightarrow \sec \mathscr{C l}(M, \mathrm{~g})$ and $B_{s} \in$ $\sec \bigwedge^{s} T^{*} M \hookrightarrow \sec \mathscr{C} \ell(M, \mathrm{~g})$. We define a scalar product in $\mathscr{C l}(M, \mathrm{~g})$ (denoted by $\cdot$ ) as follows.

(i) For $a, b \in \sec \bigwedge^{1} T^{*} M \hookrightarrow \sec \mathscr{C} \ell(M, g)$,

$$
a \cdot b=\frac{1}{2}(a b+b a)=\mathrm{g}(a, b) .
$$

(ii) For $A_{r}=a_{1} \wedge \cdots \wedge a_{r}, B_{r}=b_{1} \wedge \cdots \wedge b_{r}$ and $a_{i}, b_{j} \in$ $\sec \bigwedge^{1} T^{*} M \hookrightarrow \sec \mathscr{C} \ell(M, \mathrm{~g}), i, j=1, \ldots, r$,

$$
\begin{aligned}
A_{r} \cdot B_{r}= & \left(a_{1} \wedge \cdots \wedge a_{r}\right) \cdot\left(b_{1} \wedge \cdots \wedge b_{r}\right) \\
= & \left|\begin{array}{ccc}
a_{1} \cdot b_{1} & \cdots & a_{1} \cdot b_{r} \\
\vdots & \cdots & \vdots \\
a_{r} \cdot b_{1} & \cdots & a_{r} \cdot b_{r}
\end{array}\right| .
\end{aligned}
$$

We agree that if $r=s=0$, the scalar product is simply the ordinary product in the real field.

Also, if $r \neq s$, then $A_{r} \cdot B_{s}=0$. Finally, the scalar product is extended by linearity for all sections of $\mathscr{C} \ell(M, \mathrm{~g})$.

For $r \leq s, A_{r}=a_{1} \wedge \cdots \wedge a_{r}, B_{s}=b_{1} \wedge \cdots \wedge b_{s}$, we define the left contraction $\lrcorner:\left(A_{r}, B_{s}\right) \mapsto A_{r \underset{\mathbf{g}}{\lrcorner}} B_{s}$ by

$$
\begin{aligned}
A_{r \underset{\mathbf{g}}{\mathrm{g}} B_{s}=} & \sum_{i_{1}<\cdots<i_{r}} \epsilon^{i_{1} \cdots i_{s}}\left(a_{1} \wedge \cdots \wedge a_{r}\right) \\
& \cdot\left(b_{i_{1}} \wedge \cdots \wedge b_{i_{r}}\right)^{\sim} b_{i_{r}+1} \wedge \cdots \wedge b_{i_{s}},
\end{aligned}
$$

where $\sim$ is the reverse mapping (reversion) defined by $\sim$ : $\sec \mathscr{C} \ell(M, \mathrm{~g}) \rightarrow \sec \mathscr{C} \ell(M, \mathrm{~g})$. For any $X=\bigoplus_{p=0}^{4} X_{p}, X_{p} \in$ $\sec \bigwedge^{p} T^{*} M \hookrightarrow \sec \mathscr{C} \ell(M, \mathrm{~g})$,

$$
\widetilde{X}=\sum_{p=0}^{4} \widetilde{X}_{p}=\sum_{p=0}^{4}(-1)^{(1 / 2) k(k-1)} X_{p} .
$$

We agree that for $\alpha, \beta \in \sec \bigwedge^{0} T^{*} M$ the contraction is the ordinary (pointwise) product in the real field and that if $\alpha \in \sec \bigwedge^{0} T^{*} M, X_{r} \in \sec \bigwedge^{r} T^{*} M, Y_{s} \in \sec \bigwedge^{s} T^{*} M \hookrightarrow$ $\sec \mathscr{C} \ell(M, \mathrm{~g})$, then $\left.\left.\left(\alpha X_{r}\right)\right\lrcorner B_{s}=X_{r}\right\lrcorner\left(\alpha Y_{s}\right)$. Left contraction is extended by linearity to all pairs of sections of $\mathscr{C} \ell(M, \mathrm{~g})$; that is, for $X, Y \in \sec \mathscr{C} \ell(M, \mathrm{~g})$,

$$
X_{\breve{g}} Y=\sum_{r, s}\langle X\rangle_{r \underset{\mathbf{g}}{\lrcorner}}\langle Y\rangle_{s}, \quad r \leq s .
$$

It is also necessary to introduce the operator of right contraction denoted by $\underset{\mathrm{g}}{\mathrm{g}}$. The definition is obtained from the one presenting the left contraction with the imposition that $r \geq s$ and taking into account that now if $A_{r} \in \sec \bigwedge^{r} T^{*} M$, $B_{s} \in \sec \bigwedge^{s} T^{*} M$, then $A_{r \mathbf{g}}\left(\alpha B_{s}\right)=\left(\alpha A_{r}\right)_{\mathbf{g}} B_{s}$. See also the fourth formula in (A.9).

The main formulas used in this paper can be obtained from the following ones:

$$
\begin{aligned}
a \mathscr{B}_{s}= & a\lrcorner \mathscr{B}_{s}+a \wedge \mathscr{B}_{s}, \\
\mathscr{B}_{s} a= & \mathscr{B}_{s \underline{\mathbf{g}}} a+\mathscr{B}_{s} \wedge a, \\
a_{\mathbf{g}} \mathscr{B}_{s}= & \frac{1}{2}\left(a \mathscr{B}_{s}-(-1)^{s} \mathscr{B}_{s} a\right), \\
\mathscr{A}_{r} \mathscr{\mathscr { B }}_{s}= & (-1)^{r(s-r)} \mathscr{\mathscr { B }}_{s \underline{\mathbf{g}}} \mathscr{A}_{r}, \\
a \wedge \mathscr{B}_{s}= & \frac{1}{2}\left(a \mathscr{B}_{s}+(-1)^{s} \mathscr{B}_{s} a\right), \\
\mathscr{A}_{r} \mathscr{B}_{s}= & \left\langle\mathscr{A}_{r} \mathscr{B}_{s}\right\rangle_{|r-s|}+\left\langle\mathscr{A}_{r} \mathscr{B}_{s}\right\rangle_{|r-s|+2}+\cdots \\
& +\left\langle\mathscr{A}_{r} \mathscr{B}_{s}\right\rangle_{|r+s|}=\sum_{k=0}^{m}\left\langle\mathscr{A}_{r} \mathscr{B}_{s}\right\rangle_{|r-s|+2 k} \\
\mathscr{A}_{r} \cdot \mathscr{B}_{r}= & \mathscr{B}_{r} \cdot \mathscr{A}_{r}=\widetilde{\mathscr{A}}_{r} \mathscr{\mathbf { B }}_{r}=\mathscr{A}_{r \mathrm{~L}} \widetilde{\mathscr{B}}_{r} \\
= & \left\langle\widetilde{\mathscr{A}}_{r} \mathscr{B}_{r}\right\rangle_{0}=\left\langle\mathscr{A}_{r} \widetilde{\mathscr{B}}_{r}\right\rangle_{0} .
\end{aligned}
$$


Two other important identities used in the main text are

$$
\begin{aligned}
& \underset{\mathbf{g}}{a_{\mathbf{g}}}(\mathscr{X} \wedge \mathcal{Y})=\left(\underset{\mathbf{g}}{a_{\mathbf{g}}} \mathcal{X}\right) \wedge \mathscr{Y}+\widehat{X} \wedge\left(a_{\mathbf{g}} \mathcal{Y}\right), \\
& \mathscr{X}_{\mathbf{g}}\left(\mathscr{Y}_{\mathbf{g}} \mathscr{X}\right)=(\mathscr{X} \wedge \mathcal{Y})_{\mathbf{g}} \mathscr{E},
\end{aligned}
$$

for any $a \in \sec \bigwedge^{1} T^{*} M \hookrightarrow \mathscr{C} \ell(M, \mathrm{~g})$ and $\mathscr{X}, \mathscr{Y}, \mathscr{Z} \in$ $\sec \bigwedge T^{*} M \hookrightarrow \mathscr{C} \ell(M, \mathrm{~g})$.

A.1.1. Hodge Star Operator. Let $\underset{\mathrm{g}}{\star}$ be the Hodge star operator, that is, the mapping $\underset{\mathrm{g}}{\star}: \bigwedge^{k} T^{*} M \rightarrow \bigwedge^{4-k} T^{*} M, A_{k} \mapsto \underset{\mathrm{g}}{\star} A_{k}$. For $A_{k} \in \sec \bigwedge^{k} T^{*} M \hookrightarrow \sec \mathscr{C} \ell(M, \mathrm{~g})$ we have

$$
\begin{aligned}
{\left[B_{k} \cdot A_{k}\right] \tau_{\mathbf{g}} } & =B_{k} \wedge \underset{\mathbf{g}}{\star} A_{k} \\
& \forall B_{k} \in \sec \bigwedge^{k} T^{*} M \hookrightarrow \sec \mathscr{C l}(M, \mathrm{~g}),
\end{aligned}
$$

where $\tau_{\mathrm{g}}=\theta^{5} \in \sec \bigwedge^{4} T^{*} M \hookrightarrow \sec \mathscr{C} \ell(M, \mathrm{~g})$ is a standard volume element. We have,

$$
\underset{\mathbf{g}}{\star A_{k}}=\widetilde{A}_{k} \tau_{\mathbf{g}}=\widetilde{A}_{k} \underset{\mathbf{g}}{ } \tau_{\mathbf{g}}
$$

where as noted before, in this paper, $\widetilde{\mathscr{A}}_{k}$ denotes the reverse of $\mathscr{A}_{k}$. Equation (A.12) permits calculation of Hodge duals very easily in an orthonormal basis for which $\tau_{\mathrm{g}}=\gamma^{5}$. Let $\left\{\vartheta^{\alpha}\right\}$ be the dual basis of $\left\{e_{\alpha}\right\}$ (i.e., it is a basis for $T^{*} U \equiv \wedge^{1} T^{*} U$ ) which is either an orthonormal or a coordinate basis. Then writing $g\left(\vartheta^{\alpha}, \vartheta^{\beta}\right)=g^{\alpha \beta}$, with $g^{\alpha \beta} g_{\alpha \rho}=\delta_{\rho}^{\beta}$, and $\vartheta^{\mu_{1} \cdots \mu_{p}}=$ $\vartheta^{\mu_{1}} \wedge \cdots \wedge \vartheta^{\mu_{p}}, \mathfrak{\vartheta}^{v_{p+1} \cdots v_{n}}=\mathfrak{\vartheta}^{v_{p+1}} \wedge \cdots \wedge \mathcal{\vartheta}^{v_{n}}$ we have from (A.12)

$$
\begin{aligned}
& \stackrel{{ }_{\mathrm{g}}}{9^{\mu_{1} \cdots \mu_{p}}} \\
& =\frac{1}{(n-p) !} \sqrt{|\operatorname{det} \mathbf{g}|} g^{\mu_{1} v_{1}} \cdots g^{\mu_{p} v_{p}} \epsilon_{\nu_{1} \cdots \nu_{n}} \vartheta^{v_{p+1} \cdots v_{n}},
\end{aligned}
$$

where det $\mathbf{g}$ denotes the determinant of the matrix with entries $g_{\alpha \beta}=\mathbf{g}\left(e_{\alpha}, e_{\beta}\right)$, that is, $\operatorname{det} \mathbf{g}=\operatorname{det}\left[g_{\alpha \beta}\right]$. We also define the inverse $\star_{\mathrm{g}}^{-1}$ of the Hodge dual operator, such that

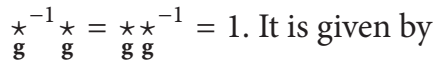

$$
\begin{aligned}
\stackrel{\star_{\mathbf{g}}^{-1}}{\mathrm{-}} \sec \bigwedge^{r} T^{*} M & \longrightarrow \sec \bigwedge^{n-r} T^{*} M, \\
& \stackrel{{ }_{\mathrm{g}}^{-1}}{-1} A_{r}=(-1)^{r(n-r)} \operatorname{sgn} \operatorname{det} \mathbf{g} \underset{\mathbf{g}}{\star} A_{r},
\end{aligned}
$$

where sgn det $\mathbf{g}=\operatorname{det} \mathbf{g} /|\operatorname{det} \mathbf{g}|$ denotes the sign of the determinant of $\mathbf{g}$.
Some useful identities (used in the text) involving the Hodge star operator, the exterior product, and contractions are

$$
\begin{aligned}
& A_{r} \wedge \underset{\mathbf{g}}{\star} B_{s}=B_{s} \wedge \underset{\mathrm{g}}{\star} A_{r} ; \quad r=s, \\
& A_{r} \cdot \underset{\mathbf{g}}{\star} B_{s}=B_{s} \cdot \underset{\mathbf{g}}{\star} A_{r} ; \quad r+s=n, \\
& A_{r} \wedge \underset{\mathbf{g}}{\star} B_{s}=(-1)^{r(s-1)} \underset{\mathbf{g}}{\star}\left(\widetilde{A}_{r \underset{\mathbf{g}}{\lrcorner}} B_{s}\right) ; \quad r \leq s, \\
& A_{r\lrcorner \mathbf{g}} \star B_{s}=(-1)^{r s} \underset{\mathbf{g}}{\star}\left(\widetilde{A}_{r} \wedge B_{s}\right) ; \quad r+s \leq n, \\
& \underset{\mathrm{g}}{\star} \tau_{\mathrm{g}}=\operatorname{sgn} \operatorname{det} \mathbf{g} ; \quad \stackrel{\star \mathrm{g}}{\star} 1=\tau_{\mathrm{g}} .
\end{aligned}
$$

A.1.2. Dirac Operator Associated with a Levi-Civita Connection D. Let $d$ and $\delta$ be, respectively, the differential and Hodge codifferential operators acting on sections of $\mathscr{C} \ell(M, \mathrm{~g})$. If $A_{p} \in \sec \bigwedge^{p} T^{*} M \hookrightarrow \sec \mathscr{C} \ell(M, \mathrm{~g})$, then $\delta_{\mathrm{g}} A_{p}=$

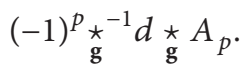

The Dirac operator acting on sections of $\mathscr{C} \ell(M, \mathrm{~g})$ associated with the metric compatible connection $D$ is the invariant first-order differential operator

$$
\partial=\vartheta^{\alpha} \mathbf{D}_{e_{\alpha}},
$$

where $\left\{e_{\alpha}\right\}$ is an arbitrary (coordinate or orthonormal) basis for $T U \subset T M$ and $\left\{\vartheta^{\alpha}\right\}$ is a basis for $T^{*} U \subset T^{*} M$ dual to the basis $\left\{e_{\alpha}\right\}$; that is, $\vartheta^{\beta}\left(e_{\alpha}\right)=\delta_{\beta}^{\alpha}, \alpha, \beta=0,1,2,3$. The reciprocal basis of $\left\{\vartheta^{\alpha}\right\}$ is denoted by $\left\{\vartheta_{\alpha}\right\}$ and we have $\vartheta_{\alpha} \cdot \vartheta_{\beta}=g_{\alpha \beta}$. Also, when $\left\{e_{\alpha}=\partial_{\alpha}\right\}$ and $\left\{\vartheta^{\alpha}=d x^{\alpha}\right\}$ we have

$$
\begin{aligned}
\mathbf{D}_{\partial_{\alpha}} \partial_{\beta} & =\Gamma_{\cdot \alpha \beta}^{\varepsilon \cdot} \partial_{\beta}, \\
\mathbf{D}_{\partial_{\alpha}} d x^{\beta} & =-\Gamma_{\cdot \alpha \varepsilon}^{\beta \cdot \cdot} d x^{\varepsilon}
\end{aligned}
$$

and when $\left\{e_{\alpha}=\mathbf{e}_{\alpha}\right\},\left\{\vartheta^{\alpha}=\gamma^{\alpha}\right\}$ are orthonormal basis we have

$$
\begin{aligned}
& \mathrm{D}_{e_{\alpha}} e_{\beta}=\omega_{\cdot \alpha \beta}^{\lambda \cdot *} e_{\lambda}, \\
& \mathbf{D}_{e_{\alpha}} \gamma^{\beta}=-\omega_{\cdot \alpha \lambda}^{\beta \cdot *} \gamma^{\lambda} .
\end{aligned}
$$

We define the connection ${ }^{38} 1$-forms in the gauge defined by $\left\{\gamma^{\alpha}\right\}$ as

$$
\omega_{\cdot \beta}^{\alpha \cdot}:=\omega_{\cdot \lambda \beta}^{\alpha \cdot} \gamma^{\lambda}
$$

Moreover, we write for an arbitrary tensor field $Y=$ $Y_{\nu_{1} \cdots \nu_{s}}^{\mu_{1} \cdots \mu_{r}} \vartheta^{\nu_{1}} \otimes \cdots \otimes \vartheta^{v_{s}} \otimes \partial_{\mu_{1}} \otimes \cdots \otimes \partial_{\mu_{r}}$ in a coordinate basis

$$
\begin{aligned}
\mathbf{D}_{\mathbf{e}_{\alpha}} Y:= & \left(\mathbf{D}_{\alpha} Y_{v_{1} \cdots \nu_{s}}^{\mu_{1} \cdots \mu_{r}}\right) \vartheta^{\nu_{1}} \otimes \cdots \otimes \vartheta^{v_{s}} \otimes \partial_{\mu_{1}} \otimes \cdots \\
& \otimes \partial_{\mu_{r}}
\end{aligned}
$$

and also when we write $Y=Y_{\nu_{1} \cdots \nu_{s}}^{\mu_{1} \cdots \mu_{r}} \boldsymbol{\gamma}^{\nu_{1}} \otimes \cdots \otimes \boldsymbol{\gamma}^{\nu_{s}} \otimes \mathbf{e}_{\mu_{1}} \otimes \cdots \otimes \mathbf{e}_{\mu_{r}}$ we also write

$$
\begin{aligned}
\mathbf{D}_{\mathbf{e}_{\alpha}} Y:= & \left(\mathbf{D}_{\alpha} Y_{\nu_{1} \cdots \nu_{s}}^{\mu_{1} \cdots \mu_{r}}\right) \gamma^{\nu_{1}} \otimes \cdots \otimes \gamma^{\nu_{s}} \otimes \mathbf{e}_{\mu_{1}} \otimes \cdots \\
& \otimes \mathbf{e}_{\mu_{r}} .
\end{aligned}
$$


So, please pay attention when reading a particular formula to certificate the meaning of $\mathbf{D}_{\alpha} Y_{v_{1} \cdots v_{s}}^{\mu_{1} \cdots \mu_{r}}$, that is, whether we are using in that formula coordinate or orthonormal frames.

We have also the important results (see, e.g., [11]) for the Dirac operator associated with the Levi-Civita connection D acting on the sections of the Clifford bundle ${ }^{39}$

$$
\begin{aligned}
\partial A_{p} & =\partial \wedge A_{p}+\partial_{\lrcorner} A_{p}=d A_{p}-\underset{\mathbf{g}}{\delta} A_{p}, \\
\partial \wedge A_{p} & =d A_{p} \\
\underset{J_{\mathbf{g}}}{A_{p}} & =-\underset{\mathbf{g}}{\delta} A_{p} .
\end{aligned}
$$

We shall need the following identity valid for any $a, b \in$ $\sec \bigwedge^{1} T^{*} M \hookrightarrow \mathscr{C} \ell(M, \mathrm{~g})$ :

$$
\begin{aligned}
\partial(a \cdot b)= & (a \cdot \partial) b+(b \cdot \partial) a+a_{\lrcorner}(\partial \wedge b) \\
& +b_{\lrcorner}(\partial \wedge a) .
\end{aligned}
$$

A.2. Covariant D'Alembertian, Hodge D'Alembertian, and Ricci Operators. The square of the Dirac operator $\diamond=\partial^{2}$ is called Hodge D'Alembertian and we have the following noticeable formulas:

$$
\begin{gathered}
\partial^{2}=-d \underset{\mathbf{g}}{\delta}-\underset{\mathbf{g}}{\delta} d, \\
\partial^{2} A_{p}=\partial \cdot \partial A_{p}+\partial \wedge \partial A_{p},
\end{gathered}
$$

where $\partial \cdot \partial$ is called the covariant D'Alembertian and $\partial \wedge \partial$ is called the Ricci operator ${ }^{40}$. If $A_{p}=(1 / p !) A_{\mu_{1} \cdots \mu_{p}} \vartheta^{\mu_{1}} \wedge \cdots \wedge \vartheta^{\mu_{p}}$, we have

$$
\begin{aligned}
\boldsymbol{\partial} \cdot \boldsymbol{\partial} A_{p} & =g^{\alpha \beta}\left(D_{\partial_{\alpha}} D_{\partial_{\beta}}-\Gamma_{\cdot \alpha \beta}^{\rho \cdot} D_{\partial_{\rho}}\right) A_{p} \\
& =\frac{1}{p !} g^{\alpha \beta} D_{\alpha} D_{\beta} A_{\alpha_{1} \cdots \alpha_{p}} \vartheta^{\alpha_{1}} \wedge \cdots \wedge \vartheta^{\alpha_{p}} .
\end{aligned}
$$

Also for $\partial \wedge \partial$ in an arbitrary basis (coordinate or orthonormal)

$$
\begin{aligned}
\partial \wedge & \partial A_{p} \\
= & \frac{1}{2} \vartheta^{\alpha} \\
& \wedge \vartheta^{\beta}\left(\left[\mathbf{D}_{e_{\alpha}}, \mathbf{D}_{e_{\beta}}\right]-\left(\Gamma_{\cdot \alpha \beta}^{\rho \cdot \cdot}-\Gamma_{\cdot \beta \alpha}^{\rho \cdot \cdot}\right) D_{e_{\rho}}\right) A_{p} .
\end{aligned}
$$

In particular we have [11]

$$
\partial \wedge \partial \vartheta^{\mu}=\mathscr{R}^{\mu}
$$

where $\mathscr{R}^{\mu}=R_{\nu}^{\mu} \vartheta^{\nu} \in \sec \bigwedge^{1} T^{*} M \hookrightarrow \sec \mathscr{C} \ell(M, g)$ are the Ricci 1-form fields, such that if $R_{\cdot v \sigma \mu}^{\mu \cdots}$ are the components of the Riemann tensor we use the convention that $R_{\nu \sigma}=R_{\cdot v \sigma \mu}^{\mu \cdots}$ are the components of the Ricci tensor.

Applying this operator to the 1-forms of the basis $\left\{\vartheta^{\mu}\right\}$, we get

$$
\partial \wedge \partial \vartheta^{\mu}=-\frac{1}{2} R_{\cdot \rho \alpha \beta}^{\mu \cdots}\left(\vartheta^{\alpha} \wedge \vartheta^{\beta}\right) \vartheta^{\rho}=\mathscr{R}_{\rho}^{\mu} \vartheta_{\rho}
$$

$\partial \wedge \partial$ is an extensor operator; that is, for $A \in$ $\sec \bigwedge^{1} T^{*} M \hookrightarrow \sec \mathscr{C} \ell(M, g)$, it is

$$
\partial \wedge \partial A=A_{\mu} \partial \wedge \partial \vartheta^{\mu}
$$

Remark A.1. We remark that covariant Dirac spinor fields used in almost all Physics texts books and research papers can be represented as certain equivalence classes of even sections of the Clifford bundle $\mathscr{C} \ell(M, \mathrm{~g})$. These objects are now called Dirac-Hestenes spinor fields (DHSF) and a thoughtful theory describing them can be found in [11, 23, 24]. Moreover, in [25], using the concept of DHSF a new approach is given to the concept of Lie derivative for spinor fields, which does not seem to have the objections of previous approaches to the subject. Of course, a meaningful definition of Lie derivative for spinor fields is a necessary condition for a formulation of conservation laws involving bosons and fermion fields in interaction in arbitrary manifolds. We will present the complete Lagrangian density involving the gravitation field (interpreted as fields in the Faraday sense and described by cotetrad fields), the electromagnetic and the DHSF living in a parallelizable manifold, and its variation in another publication.

\section{B. Lie Derivatives and Variations}

In modern field theory the physical fields are tensor and spinor fields living on a structure $\left(M, \mathbf{g}, \tau_{\mathbf{g}}, \uparrow\right)$ and interacting among themselves. Note that at this point we did not introduce any connection in our game, since according to our view (see, e.g., Chapter 11 of [11]) the introduction of a particular connection to describe Physics is only a question of convenience. For the objective of this paper we shall consider two structures (already introduced in the main text), a Lorentzian space-time $M^{\mathrm{dSL}}=\left(M, \mathbf{g}, \mathbf{D}, \tau_{\mathbf{g}}, \uparrow\right)$, where $\mathbf{D}$ is the Levi-Civita connection of $\mathbf{g}$, and a teleparallel spacetime $M^{\text {dSTP }}=\left(M, \mathbf{g}, \nabla, \tau_{\mathbf{g}}, \uparrow\right)$, where $\nabla$ is a metric compatible teleparallel connection. Minkowski space-time structure will be denoted by $\left(M, \boldsymbol{\eta}, D, \tau_{\boldsymbol{\eta}}, \uparrow\right)$. The equations of motion are derived from a variational principle once a given Lagrangian density is postulated for the interacting fields of the theory.

As is well known, diffeomorphism invariance is a crucial ingredient of any physical theory. This means that if a physical phenomenon is described by fields, say, $\phi_{1}, \ldots, \phi_{n}$ (defined in $\mathcal{U} \subset M$ ), satisfying equations of motion of the theory (with appropriated initial and boundary conditions), then if $h: M \mapsto M$ is a diffeomorphism, then the fields $h^{*} \phi_{1}, \ldots, h^{*} \phi_{N}$ (where, $h^{*}$ is the pullback mapping) describe the same physical phenomenon ${ }^{41}$ in $h \mathscr{U}$.

Suppose that fields $\phi_{1}, \ldots, \phi_{N}$ (in what follows called simply matter fields ${ }^{42}$ ) are arbitrary differential forms. Their Lagrangian density will here be defined as the functional mapping $^{43}$

$$
\begin{aligned}
\mathscr{L}_{m}:\left(\phi_{1}, \ldots, \phi_{N}, d \phi_{1}, \ldots, d \phi_{N}\right) & \longmapsto \\
\mathscr{L}_{m}(\phi, d \phi) & \in \sec \bigwedge^{4} T M,
\end{aligned}
$$


where $\mathscr{L}_{m}(\phi, d \phi)$ is here supposed to be constructed using the Hodge star operator $\star$. The action of the system is

$$
\mathscr{A}=\int_{\mathscr{U}} \mathscr{L}_{m}(\phi, d \phi)
$$

Choose a chart of $M$ covering $\mathcal{U}$ and $h \mathcal{U}$ with coordinate functions $\left\{\mathbf{x}^{\mu}\right\}$. Then under an infinitesimal mapping $h_{\varepsilon}$ : $M \mapsto M, x \mapsto x^{\prime}=h_{\varepsilon}(x)$ generated by a one-parameter group of diffeomorphisms associated with the vector field $\xi \in$ sec TM we have (with $h_{\varepsilon}^{\mu}$ being the coordinate representative of the mapping $h_{\varepsilon}$ )

$$
\begin{aligned}
x^{\mu} & =\mathbf{x}^{\mu}(x) \longmapsto x^{\prime \mu} \\
x^{\prime \mu} & =\mathbf{x}^{\mu}\left(h_{\varepsilon}(x)\right)=h_{\varepsilon}^{\mu}\left(x^{\alpha}\right)=x^{\mu}+\varepsilon \xi^{\mu},
\end{aligned}
$$

$$
|\varepsilon| \ll 1
$$

In Physics textbooks given an infinitesimal diffeomorphism $h_{\varepsilon}$ several different kinds of variations (for each one of the fields $\phi_{i}$ ) are defined.

Let $\phi$ be one of the fields $\phi_{1}, \ldots, \phi_{N}$ and recall that the Lie derivative of $\phi$ in the direction of the vector field $\xi$ is given by

$$
\mathfrak{E}_{\xi} \phi=\lim _{\varepsilon \rightarrow 0} \frac{h_{\varepsilon}^{*} \circ \phi \circ h_{\varepsilon}-\phi}{\varepsilon} .
$$

As an example, take $\phi$ as 1 -form. Then, in the chart introduced above using the definition of the pullback

$$
h_{\varepsilon}^{*} \phi_{\mu}\left(x^{\kappa}\right):=\left[h_{\varepsilon}^{*}\left(\phi\left(h_{\varepsilon}(x)\right)\right)\right]_{\mu}
$$

it is

$$
h_{\varepsilon}^{*} \phi(x)=h_{\varepsilon}^{*} \phi_{\mu}\left(x^{\kappa}\right) d x^{\mu}:=\phi_{\kappa}\left(x^{\prime \kappa}\left(x^{\kappa}\right)\right) \frac{\partial x^{\prime \kappa}}{\partial x^{\mu}} d x^{\mu} .
$$

Then, (B.4) can be written in components as

$$
\left(£_{\xi} \phi\left(x^{\kappa}\right)\right)_{\mu}=\lim _{\varepsilon \rightarrow 0} \frac{h_{\varepsilon}^{*} \phi_{\mu}\left(x^{\kappa}\right)-\phi_{\mu}\left(x^{\kappa}\right)}{\varepsilon} .
$$

Now, to first order in $\varepsilon$, we have

$$
\begin{aligned}
\frac{\partial x^{\prime \kappa}}{\partial x^{\mu}} & =\delta_{\mu}^{\kappa}+\varepsilon \partial_{\mu} \xi^{\kappa} \\
\phi_{\kappa}\left(x^{\prime \kappa}\left(x^{k}\right)\right) & =\phi_{\kappa}\left(x^{\kappa}+\varepsilon \xi^{\kappa}\right) \\
& =\phi_{\kappa}\left(x^{\kappa}\right)+\varepsilon \xi^{\alpha} \partial_{\alpha} \phi_{\kappa}\left(x^{\kappa}\right) .
\end{aligned}
$$

So,

$$
\begin{aligned}
h_{\varepsilon}^{*} \phi_{\mu}\left(x^{\kappa}\right) & =\phi_{\kappa}\left(x^{\prime \kappa}\left(x^{\kappa}\right)\right) \frac{\partial x^{\prime \kappa}}{\partial x^{\mu}} \\
& =\left(\phi_{\kappa}\left(x^{\kappa}\right)+\varepsilon \xi^{\alpha} \partial_{\alpha} \phi_{\kappa}\left(x^{\kappa}\right)\right)\left(\delta_{\mu}^{\kappa}+\varepsilon \partial_{\mu} \xi^{\kappa}\right) \\
& =\phi_{\mu}\left(x^{\kappa}\right)+\varepsilon \partial_{\mu} \xi^{\kappa} \phi_{\kappa}\left(x^{\kappa}\right)+\varepsilon \xi^{\alpha} \partial_{\alpha} \phi_{\mu}\left(x^{\kappa}\right) .
\end{aligned}
$$

Then,

$$
\left(\mathfrak{E}_{\xi} \phi\left(x^{\kappa}\right)\right)_{\mu}=\partial_{\mu} \xi^{\kappa} \phi_{\kappa}\left(x^{\kappa}\right)+\xi^{\alpha} \partial_{\alpha} \phi_{\mu}\left(x^{\kappa}\right) .
$$

Now, we define following Physics textbooks the horizontal variation $^{44}$ by

$$
\boldsymbol{\delta}^{0} \phi:=-\mathfrak{E}_{\xi} \phi
$$

This definition (with the negative sign) is used by physicists because they usually work only with the components of the fields and diffeomorphism invariance is interpreted as invariance under choice of coordinates. Then, they interpret (B.3) as a coordinate transformation between two charts whose intersection of domains cover the regions $\mathcal{U}$ and $h \mathcal{U}$ of interest with coordinate functions $\left\{\mathbf{x}^{\mu}\right\}$ and $\left\{\mathbf{x}^{\prime \mu}\right\}$ such that

$$
\mathbf{x}^{\prime \mu}:=\mathbf{x}^{\mu} \circ h_{\varepsilon}
$$

and then

$$
\mathbf{x}^{\prime \mu}(x)=x^{\mu}+\varepsilon \xi^{\mu}
$$

The field $\phi$ at $x \in \mathcal{U} \subset M$ has the representations

$$
\phi(x)=\phi_{\mu}^{\prime}\left(x^{\prime \prime}\right) d x^{\prime \mu}=\phi_{\kappa}\left(x^{l}\right) d x^{\kappa}
$$

and in first order in $\varepsilon$ it is

$$
\phi_{\mu}^{\prime}\left(x^{\prime l}\right)=\frac{\partial x^{\kappa}}{\partial x^{\prime \mu}} \phi_{\kappa}\left(x^{l}\right)=\phi_{\mu}\left(x^{l}\right)-\varepsilon \partial_{\mu} \xi^{\kappa} \phi_{\kappa}\left(x^{l}\right)
$$

and on the other hand since

$$
\phi_{\mu}^{\prime}\left(x^{\prime l}\right)=\phi_{\mu}^{\prime}\left(x^{l}+\varepsilon \xi^{l}\right)=\phi_{\mu}^{\prime}\left(x^{l}\right)+\varepsilon \xi^{\kappa} \partial_{\kappa} \phi_{\mu}^{\prime}\left(x^{l}\right)
$$

we have in first order in $\varepsilon$ that

$$
\phi_{\mu}^{\prime}\left(x^{l}\right)=\phi_{\mu}\left(x^{l}\right)-\varepsilon \partial_{\mu} \xi^{\kappa} \phi_{\kappa}\left(x^{l}\right)-\varepsilon \xi^{\kappa} \partial_{\kappa} \phi_{\mu}\left(x^{l}\right)
$$

from where we get

$$
\delta^{0} \phi_{\mu}(x)=\lim _{\varepsilon \rightarrow 0} \frac{\phi_{\mu}^{\prime}\left(x^{\kappa}\right)-\phi_{\mu}\left(x^{\kappa}\right)}{\varepsilon}=-\left(£_{\xi} \phi\left(x^{\kappa}\right)\right)_{\mu} .
$$

Remark B.1. The above calculation can be done in a while recalling Cartan's "magical" formula, which with $\xi:=\mathbf{g}(\boldsymbol{\xi}$, reads

$$
£_{\xi} \phi=\xi_{\lrcorner} d \phi+d\left(\xi_{\lrcorner} \phi\right) .
$$

In components we have

$$
\begin{aligned}
\left(\xi_{\lrcorner} d \phi\right)_{\alpha} & =\xi^{\mu} \partial_{\mu} \phi_{\alpha}-\xi^{\mu} \partial_{\alpha} \phi_{\mu}, \\
\left(d\left(\xi_{\lrcorner} \phi\right)\right)_{\alpha} & =\partial_{\alpha} \xi^{\mu} \phi_{\mu}+\xi^{\mu} \partial_{\alpha} \phi_{\mu}
\end{aligned}
$$

from which the substituting of these results in (B.19) and (B.10) follows immediately.

Remark B.2. If we have chosen the coordinate functions $\left\{\mathbf{x}^{\mu}\right\}$ and $\left\{\mathbf{x}^{\prime \mu}\right\}$ related by ${ }^{45}$

$$
\mathbf{x}^{\prime \mu}:=\mathbf{x}^{\mu} \circ h_{\varepsilon}^{-1}
$$

we would get that $\delta^{0} \phi_{\mu}(x)=\left(\mathfrak{E}_{\xi} \phi\left(x^{\kappa}\right)\right)_{\mu}$. 
Remark B.3. Take into account for applications that for any $\mathscr{C} \in \sec \bigwedge T^{*} M$

$$
d £_{\xi} \mathscr{C}=£_{\xi} d \mathscr{C}
$$

Now, physicists introduce another two variations $\boldsymbol{\delta}^{a}$ and $\boldsymbol{\delta}$ defined by

$$
\begin{aligned}
\boldsymbol{\delta}^{a} \phi_{\mu}(x) & :=\lim _{\varepsilon \rightarrow 0} \frac{\phi_{\mu}\left(x^{\prime \kappa}\right)-\phi_{\mu}\left(x^{\kappa}\right)}{\varepsilon}=\xi^{\iota} \partial_{\iota} \phi_{\mu}\left(x^{\kappa}\right), \\
\delta \phi_{\mu}(x) & :=\lim _{\varepsilon \rightarrow 0} \frac{\phi_{\mu}^{\prime}\left(x^{\prime \kappa}\right)-\phi_{\mu}\left(x^{\kappa}\right)}{\varepsilon}
\end{aligned}
$$

called, for example, in [26], local variation ${ }^{46}$. We have

$$
\begin{aligned}
(\boldsymbol{\delta} \phi(x))_{\mu}= & \lim _{\varepsilon \rightarrow 0} \frac{\phi_{\mu}^{\prime}\left(x^{\kappa}\right)+\varepsilon \xi^{l} \partial_{\iota} \phi_{\mu}\left(x^{\kappa}\right)-\phi_{\mu}\left(x^{\kappa}\right)}{\varepsilon} \\
= & \left(\boldsymbol{\delta}^{0} \phi\left(x^{\kappa}\right)\right)_{\mu}+\xi^{l} \partial_{\iota} \phi_{\mu}\left(x^{\kappa}\right) \\
= & -\partial_{\mu} \xi^{l} \phi_{l}\left(x^{\kappa}\right)-\xi^{\alpha} \partial_{\alpha} \phi_{\mu}\left(x^{\kappa}\right) \\
& +\xi^{l} \partial_{\iota} \phi_{\mu}\left(x^{\kappa}\right)=-\partial_{\mu} \xi^{l} \phi_{l}\left(x^{\kappa}\right) .
\end{aligned}
$$

Remark B.4. In what follows we shall use the above terminology for the various variations introduced above for an arbitrary tensor field. The definition of the Lie derivative of spinor fields is still a subject of recent research with many conflicting views. In [25] we present a novel geometrical approach to this subject using the theory of Clifford and spinClifford bundles which seems to lead to consistent results.

Remark B.5. Defining

$$
\boldsymbol{\delta}^{0} \mathscr{A}:=-\int_{\mathscr{U}} \mathfrak{E}_{\xi} \mathscr{L}_{m}(\phi, d \phi),
$$

we have returning to (B.2) that Stokes theorem permit us to write

$$
\begin{aligned}
\boldsymbol{\delta}^{0} \mathscr{A}= & -\int_{\mathscr{U}} \mathfrak{E}_{\xi} \mathscr{L}_{m}(\phi, d \phi) \\
= & -\int_{\mathscr{U}} d\left(\xi_{\lrcorner} \mathscr{L}_{m}(\phi, d \phi)\right) \\
& -\int_{\mathscr{U}} \xi_{\lrcorner} d \mathscr{L}_{m}(\phi, d \phi) \\
= & -\int_{\partial \mathscr{U}} \xi_{\lrcorner} \mathscr{L}_{m}(\phi, d \phi) .
\end{aligned}
$$

\section{The Generalized Energy-Momentum Current in $\left(M, \mathbf{g}, \tau_{\mathbf{g}}, \uparrow\right)$}

C.1. The Case of a General Lorentzian Space-Time Structure. In this subsection $\left(M, \mathbf{g}, \tau_{\mathbf{g}}, \uparrow\right)$ is an arbitrary oriented and time-oriented Lorentzian manifold $(M, \mathbf{g})$ which will be supposed to be the arena where physical phenomena take place. We choose coordinate charts $\left(\mathscr{U}_{1}, \chi_{1}\right)$ and $\left(\mathscr{U}_{2}, \chi_{2}\right)$ with coordinate functions $\left\{\mathbf{x}^{\mu}\right\}$ and $\left\{\mathbf{x}^{\prime \mu}\right\}$ covering $\mathcal{U}_{1} \cap \mathcal{U}_{2}=\mathscr{U}$. We call $\chi_{1}(\mathcal{U})=U, \chi_{2}(\mathcal{U})=U^{\prime}$. We take $\mathcal{U}$ such that $\partial \mathcal{U}=\Sigma_{2}-\Sigma_{1}+\boldsymbol{\Xi}$, that is, $\mathcal{U}$ is bounded from above and below by spacelike surfaces $\Sigma_{1}$ and $\Sigma_{2}$ such that $\sigma_{1}=\chi_{1}\left(\Sigma_{1}\right)$ and $\sigma_{2}=\chi_{1}\left(\Sigma_{2}\right)$, and moreover we suppose that set of the $N$ matter fields in interaction denoted by

$$
\phi=\left\{\phi_{A}\right\}, \quad A=1,2, \ldots, N
$$

living in $\mathcal{U}$ satisfy in $\Xi$ (a timelike boundary)

$$
\left.\phi_{A}\right|_{\Xi}=0 .
$$

In what follows the action functional for the fields is written

$$
\begin{aligned}
\mathscr{A} & =\int_{\mathscr{U}} \mathfrak{Q}_{m}\left(\phi_{\mu}, \partial_{\mu} \phi\right) d^{4} x \\
& =\int_{\mathscr{U}} L_{m}\left(\phi_{\mu}, \partial_{\mu} \phi\right) \sqrt{-\operatorname{det} \mathbf{g}} d^{4} x .
\end{aligned}
$$

Under a coordinate transformation corresponding to a diffeomorphism generated by a one-parameter group of diffeomorphisms,

$$
\begin{aligned}
x^{\mu} & \longmapsto \\
x^{\prime \mu} & =x^{\mu}+\varepsilon \xi^{\mu}=x^{\mu}+\varepsilon \xi\left[x^{\mu}\right]=x^{\mu}-\mathfrak{E}_{\varepsilon \xi} x^{\mu} \\
& =x^{\mu}+\delta x^{\mu},
\end{aligned}
$$

we already know that the fields suffer the variation

$$
\phi \longmapsto \phi^{\prime}=\phi+\delta^{0} \phi .
$$

We have in first order in $\varepsilon$, recalling that $\delta^{0}$ and $\partial_{\mu}$ commute, that

$$
\begin{aligned}
& \boldsymbol{\delta}^{0} \mathscr{A}=\int_{\mathscr{U}} \mathfrak{R}_{m}\left(\phi_{\mu}^{\prime}, \partial_{\mu} \phi^{\prime}\right) d^{4} x^{\prime} \\
& -\int_{\mathcal{U}} \mathfrak{R}_{m}\left(\phi_{\mu}, \partial_{\mu} \phi\right) d^{4} x=\int_{\mathscr{U}}\left(\frac{\partial \mathfrak{Q}_{m}}{\partial \phi_{A}} \boldsymbol{\delta}^{0} \phi_{A}\right. \\
& \left.+\frac{\partial \boldsymbol{Q}_{m}}{\partial \partial_{\mu} \phi_{A}} \partial_{\mu} \boldsymbol{\delta}^{0} \phi_{A}+\mathfrak{\Omega}_{m} \frac{\partial \delta x^{\mu}}{\partial x^{\mu}}\right) d^{4} x \\
& =\int_{\mathscr{U}}\left(\frac{\partial \mathfrak{Q}_{m}}{\partial \phi_{A}}-\partial_{\mu} \frac{\partial \mathfrak{Q}_{m}}{\partial \partial_{\mu} \phi_{A}}\right) \boldsymbol{\delta}^{0} \phi_{A}+\partial_{\mu}\left(\frac{\partial \mathfrak{Q}_{m}}{\partial \partial_{\mu} \phi_{A}}\right. \\
& \left.\boldsymbol{\delta}^{0} \phi_{A}+\mathfrak{\Omega}_{m} \delta x^{\mu}\right) d^{4} x \text {. }
\end{aligned}
$$

Putting

$$
\mathcal{g}^{\mu}:=\frac{\partial \boldsymbol{Q}_{m}}{\partial \partial_{\mu} \phi_{A}} \boldsymbol{\delta}^{0} \phi_{A}+\mathfrak{Q}_{m} \boldsymbol{\delta} x^{\mu}
$$

the second term in (C.6) can be written using Gauss theorem as

$$
\begin{gathered}
\int_{\mathscr{U}} \partial_{\mu}\left(\frac{\partial \mathfrak{Q}_{m}}{\partial \partial_{\mu} \phi_{A}} \boldsymbol{\delta}^{0} \phi_{A}+\mathfrak{Q}_{m} \delta x^{\mu}\right) d^{4} x \\
=\int_{\sigma_{2}} \mathcal{J}^{\mu} d \sigma_{\mu}-\int_{\sigma_{1}} \mathcal{J}^{\mu} d \sigma_{\mu} .
\end{gathered}
$$


Recalling the concept of local variation introduced above we have

$$
\delta \phi_{A}=\boldsymbol{\delta}^{0} \phi_{A}+\delta x^{\nu} \partial_{\nu} \phi_{A}
$$

Putting

$$
\pi_{A}^{\mu}=\frac{\partial \mathfrak{Q}_{m}}{\partial \partial_{\mu} \phi_{A}}
$$

we call

$$
\pi_{A}:=\pi_{A}^{\mu} \partial_{\mu}
$$

the canonical momentum canonically conjugated to the field $\phi_{A}$. Moreover, putting

$$
\Upsilon_{\nu}^{\mu}:=\pi_{A}^{\mu} \partial_{\nu} \phi_{A}-\delta_{\nu}^{\mu} \boldsymbol{\Omega}_{m}
$$

we call

$$
\Upsilon:=\Upsilon_{\nu}^{\mu} d x^{\nu} \otimes \partial_{\mu}
$$

the canonical energy-momentum tensor of the closed physical system described by the fields.

Now, we can write (C.7) as

$$
\mathcal{J}^{\mu}:=\pi_{A}^{\mu} \delta \phi_{A}-\Upsilon_{\nu}^{\mu} \delta x^{\nu}
$$

Moreover, defining

$$
F(\sigma):=\int_{\sigma}\left(\pi_{A}^{\mu} \delta \phi_{A}-\Upsilon_{\nu}^{\mu} \delta x^{\nu}\right) d \sigma_{\mu}
$$

we can rewrite (C.6):

$$
\begin{aligned}
\boldsymbol{\delta}^{0} \mathscr{A}= & \int_{U}\left(\frac{\partial \mathfrak{\Omega}_{m}}{\partial \phi_{A}}-\partial_{\mu} \frac{\partial \mathfrak{\Omega}_{m}}{\partial \partial_{\mu} \phi_{A}}\right) \boldsymbol{\delta}^{0} \phi_{A} d^{4} x+F\left(\sigma_{2}\right) \\
& -F\left(\sigma_{1}\right) .
\end{aligned}
$$

Now, the action principle establishes that $\boldsymbol{\delta}^{0} \mathscr{A}=0$ and then we must have

$$
\frac{\partial \boldsymbol{Q}_{m}}{\partial \phi_{A}}-\partial_{\mu} \frac{\partial \boldsymbol{Q}_{m}}{\partial \partial_{\mu} \phi_{A}}=0
$$

which are the Euler-Lagrange equations satisfied by each one of the fields $\phi_{A}$ and also

$$
F\left(\sigma_{2}\right)-F\left(\sigma_{1}\right)=0
$$

Now, if $\tau_{\mathbf{g}}$ is the volume element, taking into account that we took $\Sigma_{2}-\Sigma_{1}+\Xi=\partial \mathcal{U}$ where $\Xi$ is a timelike surface such that $\left.\mathscr{J}\right|_{\Xi}=0$ and introducing the current

$$
\mathscr{J}:=\mathscr{J}_{\mu} d x^{\mu}=g_{\mu \nu} \mathscr{F}^{\nu} d x^{\mu} \in \sec \bigwedge^{1} T^{*} M \hookrightarrow \sec \mathscr{C} \ell(M, \mathrm{~g}),
$$

we can rewrite (C.18) using Stokes theorem as

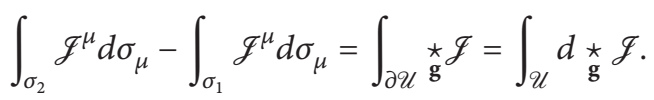

C.2. Introducing $\mathbf{D}$ and the Covariant "Conservation" Law for $\mathbf{Y}$. If we add $\mathbf{D}$, the Levi-Civita connection of $\mathbf{g}$ to $\left(M, \mathbf{g}, \tau_{\mathbf{g}}, \uparrow\right)$, we get a Lorentzian space-time structure $\left(M, \mathbf{g}, \mathbf{D}, \tau_{\mathbf{g}}, \uparrow\right)$. Then recalling from Appendix A the definitions of the Hodge coderivative and of the Dirac operator we can write

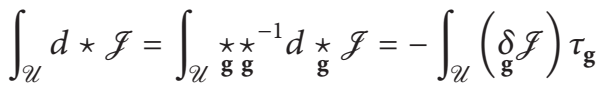

$$
\begin{aligned}
& \left.=\int_{\mathscr{U}} \partial\right\lrcorner \mathscr{J}_{\mathbf{g}}=\int_{\mathscr{U}} \mathbf{D}_{\mu} \mathscr{J}^{\mu} \tau_{\mathbf{g}}
\end{aligned}
$$

and we arrive at the conclusion that $\boldsymbol{\delta}^{0} \mathscr{A}=0$ implies that

$$
\begin{aligned}
& d \underset{\mathbf{g}}{\star} \mathscr{J}=0 \Longleftrightarrow \\
& \mathbf{D}_{\mu} \mathcal{J}^{\mu}=0 \Longleftrightarrow \\
& \frac{1}{\sqrt{-\operatorname{det} \mathbf{g}}} \partial_{\mu}\left(\sqrt{-\operatorname{det} \mathbf{g} \mathscr{J}^{\mu}}\right)=0 .
\end{aligned}
$$

Remark C.1. Recalling the definition of the canonical energymomentum tensor $\Upsilon$ (see (C.13)) gives a covariant "conservation" law for $\Upsilon$, that is,

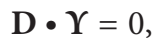

only if the term $\pi_{A}^{\mu} \delta \phi_{A}$ in the current $J^{\mu}$ is null. This, of course, happens if the local variation of the fields $\delta \phi_{A}=$ 0 , something that cannot happen in an arbitrary structure $\left(M, \mathbf{g}, \tau_{\mathbf{g}}, \uparrow\right)$. So, we need to investigate when this occurs.

Remark C.2. We observe here that comparison of (C.16) with (B.26) permits us to write

$$
\begin{aligned}
\int_{\partial \mathscr{U}} \stackrel{\mathrm{g}}{\star} \mathscr{J} & \left.=\int_{\mathscr{U}} d \underset{\mathbf{g}}{\star} \mathscr{J}=-\int_{\partial \mathscr{U}} \xi\right\lrcorner \mathscr{L}(\phi, d \phi) \\
& \left.=-\int_{\mathscr{U}} d(\xi\lrcorner \mathscr{L}(\phi, d \phi)\right) .
\end{aligned}
$$

\section{C.3. The Case of Minkowski Space-Time}

C.3.1. The Canonical Energy-Momentum Tensor in Minkowski Space-Time. We now apply the results of the last section to the case where the fields live in Minkowski spacetime $\left(M, \boldsymbol{\eta}, D, \tau_{\boldsymbol{\eta}}, \uparrow\right)$. In this case we can introduce global coordinates $\left\{x^{\mu}\right\}$ in Einstein-Lorentz-Poincaré gauge (see Section 2.3).

We now construct the conserved current associated with the diffeomorphisms generated by the vector fields $e_{\mu}=$ $\partial / \partial x^{\mu}$ which are Killing vector fields on $(M, \boldsymbol{\eta})$. Consider then the Killing vector field

$$
\xi:=\varepsilon^{\mu} e_{\mu}
$$

where $\varepsilon^{\mu} \in \mathbb{R}$ are constants such that $\left|\varepsilon^{\mu}\right| \ll 1$ and the coordinate transformation

$$
\begin{aligned}
x^{\mu} & \longmapsto x^{\prime \mu} \\
x^{\prime \mu} & =x^{\mu}+\mathcal{E}_{\xi} x^{\mu}=x^{\mu}+\varepsilon^{\mu} .
\end{aligned}
$$


Recalling the definitions of $\pi_{A}$, the momentum canonically conjugated to the field $\phi_{A}$ (see (C.11)) and of $\Upsilon$ (see (C.13)) and recalling that in the present case it is $\delta \phi_{A}=0$ we have that the conserved Noether current is

$$
\mathscr{g}^{\mu}=-\varepsilon^{\nu} \Upsilon_{v}^{\mu}
$$

and the canonical energy-momentum tensor of the physical system described by the fields $\phi_{A}$ is conserved; that is,

$$
\partial_{\mu} Y_{\nu}^{\mu}=0
$$

Remark C.3. Of course, if we introduce an arbitrary coordinate system $\left\{x^{\mu}\right\}$ covering an open set $\mathscr{U}$ of the Minkowski space-time manifold $M \simeq \mathbb{R}^{4}$, (C.28) reads

$$
\begin{aligned}
& D \bullet \Upsilon=0 \Longleftrightarrow \\
& D_{\mu} \Upsilon_{\nu}^{\mu}=0 .
\end{aligned}
$$

C.3.2. The Energy-Momentum 1-Form Fields in Minkowski Space-Time. Recall that the objects

$$
\Upsilon^{\mu}:=\left(\pi_{A}^{\mu} \partial_{\nu} \phi_{A}-\delta_{\nu}^{\mu} \ell\right) d x^{\nu}=\Upsilon_{\nu}^{\mu} d x^{\nu} \in \sec \bigwedge^{1} T^{*} M \hookrightarrow \sec \mathscr{C} \ell(M, \eta)
$$

are conserved currents. They may be called the generalized energy-momentum 1-form fields of the physical system described by the fields $\phi_{A}$. We have

$$
\begin{aligned}
& \delta \Upsilon_{\eta}^{\mu}=0 \Longleftrightarrow \\
& d \underset{\eta}{\star} \mathbf{Y}^{\mu}=0 .
\end{aligned}
$$

C.3.3. The Belinfante Energy-Momentum Tensor in Minkowski Space-Time. It happens that given an arbitrary field theory the canonical energy-momentum tensor is in general not symmetric, that is,

$$
\Upsilon^{\mu \nu} \neq \Upsilon^{\nu \mu} .
$$

But, of course, if $\Upsilon^{\mu}$ is conserved, so it is

$$
\mathscr{T}^{\mu}:=\mathfrak{Y}^{\mu}+\boldsymbol{F}^{\mu},
$$

where

$$
\boldsymbol{F}^{\mu}:=\delta_{\eta} \Psi^{\mu}
$$

with each one of the $\Psi^{\mu} \in \sec \bigwedge^{2} T^{*} M \hookrightarrow \sec \mathscr{C} \ell(M, g)$. So, it is always possible for any field theory to find ${ }^{47}$ a condition on the $\Psi^{\mu}$ such that the components $T_{\nu}^{\mu}$ of $\mathscr{T}^{\mu}=T_{\nu}^{\mu} d x^{\nu}$ satisfy the symmetry condition.

$$
T^{\mu \nu}=T^{\nu \mu} .
$$

When this is the case $\mathbf{T}=T_{\nu}^{\mu} d x^{\nu} \otimes \partial_{\mu}$ will be called the Belinfante energy-momentum tensor of the system.

C.3.4. The Energy-Momentum Covector in Minkowski SpaceTime. Since Minkowski space-time is parallelizable we can identify all tangent and cotangent spaces and thus define a covector in a vector space $\mathscr{V} \simeq \mathbb{R}^{4}$. Fixing (global) coordinates in Einstein-Lorentz-Poincaré gauge $\left\{x^{\mu}\right\}$ a vector $\mathbf{v}_{x} \in T_{x} M$ can be identified by a pair [27] $(\mathbf{x}, \mathbf{v})$, where $(\mathbf{x}, \mathbf{v}) \in \mathbb{R}^{4} \times \mathbb{R}^{4}$ and $\mathbf{x}=\left(x^{0}, x^{1}, x^{2}, x^{3}\right)$. If two vectors $\mathbf{v}_{x} \in T_{x} M, \mathbf{v}_{y} \in T_{y} M$ are such that

$$
\begin{aligned}
& \mathbf{v}_{x}=(\mathbf{x}, \mathbf{v}), \\
& \mathbf{v}_{y}=(\mathbf{y}, \mathbf{v}),
\end{aligned}
$$

that is, they have the same vector part, we will say that they can be identified as a a vector of some vector space $\mathbf{V} \simeq \mathbb{R}^{4}$. With these considerations we write $\forall x, y \in M \simeq \mathbb{R}^{4}$

$$
\begin{aligned}
& \left.\left.\frac{\partial}{\partial x^{\mu}}\right|_{x} \approx \frac{\partial}{\partial x^{\mu}}\right|_{y}=\mathbf{E}_{\mu}, \\
& \left.\left.d x^{\mu}\right|_{x} \approx d x^{\mu}\right|_{y}=\mathbf{E}^{\mu},
\end{aligned}
$$

where $\left\{\mathbf{E}_{\mu}\right\}$ is a basis of $\mathbf{V}$ and $\left\{\mathbf{E}^{\mu}\right\}$ is a basis of a $\mathscr{V} \simeq \mathbb{R}^{4}$. Then we can write (with $o \in M$ an arbitrary point, taken in general, for convenience as origin of the coordinate system)

$$
\mathbf{P}=P_{\mu} \mathbf{E}^{\mu}=\left.P_{\mu} d x^{\mu}\right|_{0}:=\left.\left(\int_{\eta}^{\underset{\eta}{T}} \mathscr{T}_{\mu}\right) d x^{\mu}\right|_{0}
$$

as the energy-momentum covector of the closed physical system described by the fields $\phi_{A}$.

Remark C.4. Note that under a global (constant) Lorentz transformation $\partial / \partial x^{\mu} \mapsto \partial / \partial x^{\prime \mu}=\Lambda_{\mu}^{\nu} \partial / \partial x^{\nu}$ we have that $\underset{\eta}{\star} \mathscr{T}_{\mu} \mapsto \underset{\eta}{\star} \mathscr{T}_{\mu}^{\prime}=\underset{\eta}{\star} \mathscr{T}_{\nu} \Lambda_{\mu}^{\nu}$ and it results in $P_{\mu} \mapsto P_{\mu}^{\prime}=P_{\nu} \Lambda_{\mu}^{\nu}$; that is, $P_{\mu}$ are indeed the components of a covector under any global (constant) Lorentz transformation.

\section{The Energy-Momentum Tensor of Matter in GRT}

The result of the previous section shows that in a general Lorentzian space-time structure the canonical Lagrangian formalism does not give a covariant "conserved" energymomentum tensor unless the local variations of the matter fields $\boldsymbol{\delta} \phi_{A}$ are null. So, in GRT the matter energy-momentum tensor $\mathbf{T}=T_{\nu}^{\mu} d x^{\nu} \otimes \partial / \partial x^{\mu}$ that enters Einstein equation is symmetric (i.e., $T^{\mu \nu}=T^{\nu \mu}$ ) and it is obtained in the following way. We start with the matter action

$$
\mathscr{A}_{m}=\int_{\mathscr{U}} L_{m}\left(\phi_{\mu}, \partial_{\mu} \phi\right) \sqrt{-\operatorname{det} \mathbf{g}} d^{4} x .
$$

Remark D.1. Consider, as above, a diffeomorphism $x \mapsto x^{\prime}=$ $h_{\varepsilon}(x)$ generated by a one-parameter group associated with 
a vector field $\xi=\xi^{\mu} \partial_{\mu}$ (such that components $\left|\xi^{\mu}\right| \ll 1$ and $\xi^{\mu} \rightarrow 0$ at $\Xi$ ) and a corresponding coordinate transformation

$$
\begin{gathered}
x^{\mu} \longmapsto x^{\prime \mu} \\
x^{\prime \mu}=x^{\mu}+\varepsilon \xi^{\mu}
\end{gathered}
$$

with $|\varepsilon| \ll 1$ and study the variation of $\mathscr{A}_{m}$ that induced the variation of the (gravitational) field $\mathbf{g}$ (without changing the fields $\phi_{A}$ ) induced by the coordinate transformation of (D.2). We have immediately that

$$
\begin{aligned}
g^{\mu \nu}\left(x^{\kappa}\right) \longmapsto & g^{\prime \mu \nu}\left(x^{\prime \kappa}\right) \\
g^{\prime \mu \nu}\left(x^{\prime \kappa}\right)= & g^{\mu \nu}\left(x^{\kappa}\right)+\varepsilon g^{\mu l}\left(x^{\kappa}\right) \partial_{\iota} \xi^{\nu} \\
& +\varepsilon g^{\nu l}\left(x^{\kappa}\right) \partial_{\iota} \xi^{\mu} .
\end{aligned}
$$

To first order in $\varepsilon$ it is

$$
\begin{aligned}
g^{\prime \mu \nu}\left(x^{\kappa}\right)= & g^{\mu \nu}\left(x^{\kappa}\right)+\varepsilon g^{\mu l}\left(x^{\kappa}\right) \partial_{\iota} \xi^{\nu}+\varepsilon g^{\nu l}\left(x^{\kappa}\right) \partial_{l} \xi^{\mu} \\
& -\varepsilon \xi^{\iota} \partial_{\iota} g^{\mu \nu} \\
\boldsymbol{\delta}^{0} g^{\mu \nu}= & -\mathfrak{E}_{\xi} g^{\mu \nu}=\mathbf{D}^{\nu} \xi^{\mu}+\mathbf{D}^{\mu} \xi^{\nu} \\
\boldsymbol{\delta}^{0} g_{\mu \nu}= & -\mathfrak{E}_{\xi} g_{\mu \nu}=-\mathbf{D}_{\nu} \xi_{\mu}-\mathbf{D}_{\mu} \xi_{\nu} .
\end{aligned}
$$

Then, under the above conditions, using Gauss theorem and supposing that $\boldsymbol{\delta}^{0} g^{\mu \nu}$ vanishes at $\boldsymbol{\Xi}$, it is

$$
\begin{aligned}
& \boldsymbol{\delta}^{0} \mathscr{A}_{m}:=\int_{\mathcal{U}}\left\{\begin{array}{c}
\frac{\partial L_{m} \sqrt{-\operatorname{det} \mathbf{g}}}{\partial g^{\mu \nu}} \boldsymbol{\delta}^{0} g^{\mu \nu} \\
+\frac{\partial L_{m} \sqrt{-\operatorname{det} \mathbf{g}}}{\partial \partial_{\iota} g^{\mu \nu}} \delta^{0}\left(\partial_{\iota} g^{\mu \nu}\right)
\end{array}\right\} d^{4} x \\
& =\int_{\mathcal{U}}\left\{\begin{array}{c}
\frac{\partial L_{m} \sqrt{-\operatorname{det} \mathbf{g}}}{\partial g^{\mu \nu}} \boldsymbol{\delta}^{0} g^{\mu \nu} \\
+\frac{\partial L_{m} \sqrt{-\operatorname{det} \mathbf{g}}}{\partial \partial_{\iota} g^{\mu \nu}} \partial_{\iota} \boldsymbol{\delta}^{0} g^{\mu \nu}
\end{array}\right\} d^{4} x \\
& =\int_{\mathscr{U}}\left\{\begin{array}{c}
\frac{\partial L_{m} \sqrt{-\operatorname{det} \mathbf{g}}}{\partial g^{\mu \nu}} \\
-\frac{\partial}{\partial x^{l}}\left(\frac{\partial L_{m} \sqrt{-\operatorname{det} \mathbf{g}}}{\partial \partial_{\iota} g^{\mu \nu}}\right)
\end{array}\right\} \boldsymbol{\delta}^{0} g^{\mu \nu} d^{4} x \\
& =: \frac{1}{2} \int_{\mathcal{U}} T_{\mu \nu} \boldsymbol{\delta}^{0} g^{\mu \nu} \sqrt{-\operatorname{det} \mathbf{g}} d^{4} x \\
& =-\frac{1}{2} \int_{\mathscr{U}} T^{\mu v} \boldsymbol{\delta}^{0} g_{\mu \nu} \sqrt{-\operatorname{det} \mathbf{g}} d^{4} x
\end{aligned}
$$

with

$$
\begin{aligned}
\frac{1}{2} \sqrt{-\operatorname{det} \mathbf{g}} T_{\mu \nu}:= & \frac{\partial L_{m} \sqrt{-\operatorname{det} \mathbf{g}}}{\partial g^{\mu \nu}} \\
& -\frac{\partial}{\partial x^{\iota}}\left(\frac{\partial L_{m} \sqrt{-\operatorname{det} \mathbf{g}}}{\partial \partial_{\iota} g^{\mu \nu}}\right) .
\end{aligned}
$$

Since $T_{\mu \nu}=T_{\nu \mu}$ we can write

$$
\begin{aligned}
\boldsymbol{\delta}^{0} \mathscr{A}_{m}= & \frac{1}{2} \int_{\mathscr{U}} T_{\mu \nu} \boldsymbol{\delta}^{0} g^{\mu \nu} \sqrt{-\operatorname{det} \mathbf{g}} d^{4} x \\
= & \frac{1}{2} \int_{\mathscr{U}} T^{\mu \nu}\left(\mathbf{D}_{\nu} \xi_{\mu}+\mathbf{D}_{\mu} \xi_{\nu}\right) \sqrt{-\operatorname{det} \mathbf{g}} d^{4} x \\
= & \int_{\mathcal{U}} T^{\mu \nu} \mathbf{D}_{\nu} \xi_{\mu} \sqrt{-\operatorname{det} \mathbf{g}} d^{4} x \\
= & \int_{\mathscr{U}} \mathbf{D}_{\nu}\left(T_{\mu}^{v} \xi^{\mu} \mu\right) \sqrt{-\operatorname{det} \mathbf{g}} d^{4} x \\
& -\int_{\mathscr{U}}\left(\mathbf{D}_{\nu} T_{\mu}^{\nu}\right) \xi^{\mu} \sqrt{-\operatorname{det} \mathbf{g}} d^{4} x \\
= & \int_{\mathscr{U}} \partial_{\nu}\left(\sqrt{-\operatorname{det} \mathbf{g}} T_{\mu}^{\nu} \xi^{\mu} \mu\right) d^{4} x \\
& -\int_{\mathscr{U}}\left(\mathbf{D}_{\nu} T_{\mu}^{\nu}\right) \xi^{\mu} \sqrt{-\operatorname{det} \mathbf{g}} d^{4} x \\
= & -\int_{\mathscr{U}}\left(\mathbf{D}_{\nu} T_{\mu}^{\nu}\right) \xi^{\mu} \sqrt{-\operatorname{det} \mathbf{g}} d^{4} x .
\end{aligned}
$$

Remark D.2. Contrary to what is stated in many textbooks in GRT we cannot conclude with the ingredients introduced in this section that $\boldsymbol{\delta}^{0} \mathscr{A}_{m}=0$.

However, if we take into account that in GRT the total action describing the mater fields and the gravitational field is

$$
\mathscr{A}=\int \mathscr{L}_{g}+\int \mathscr{L}_{m}=-\frac{1}{2} \int R \boldsymbol{\tau}_{\mathrm{g}}+\int \mathscr{L}_{m}
$$

we get from the variation $\delta^{0} \mathscr{A}$ induced by the variation of the (gravitational) field $\mathbf{g}$ (without changing the fields $\phi_{A}$ ) and induced by the coordinate transformation given by (D.2) the Einstein field equation $\mathbf{G}=-\mathbf{T}$ which reads in components as

$$
G_{v}^{\mu}=R_{v}^{\mu}-\frac{1}{2} R \delta_{v}^{\mu}=-T_{v}^{\mu}
$$

Since it is $\mathbf{D} \cdot \mathbf{G}=0$ it follows that in GRT we have

$$
\mathrm{D} \cdot \mathrm{T}=0 .
$$

Remark D.3. It is opportune to recall that as observed, for example, by Weinberg [28] for the case of Minkowski spacetime the symmetric energy-momentum tensor obtained by the above method is always equal to a convenient symmetrization of the canonical energy-momentum tensor. But it is necessary to have in mind that the GRT procedure eliminates a legitimate conserved current $\mathscr{F}$ introducing a covariant "conserved" energy-momentum tensor that does not give any legitimate energy-momentum conserved current for the matter fields, except for the particular Lorentzian space-times containing appropriate Killing vector fields. And even in this case no energy-momentum covector as it exists in special relativistic theories can be defined. Moreover, at this point we cannot forget the existence of the quantum 
structure of matter fields which experimentally says that the Minkowskian concept of energy and momentum is carried out by field excitations that one calls particles. This strongly suggests that, parodying (again) Sachs and Wu [4], it is really a shame to lose the special relativistic conservations laws in GRT.

\section{E. Relative Tensors and Their Covariant Derivatives}

Now, recall that given arbitrary coordinates $\left\{x^{\alpha}\right\}$ covering $U \subset M$ and $\left\{x^{\prime \alpha}\right\}$ covering $V \subset M(U \cap V \neq \varnothing)$ a relative tensor $\mathfrak{A}$ of type $(r, s)$ and weight ${ }^{48} w$ is a section of the bundle ${ }^{49} T_{q}^{p} M \otimes\left(\bigwedge^{4} T^{*} M\right)^{\otimes w}$.

We have

$$
\begin{aligned}
\mathfrak{A}= & \mathfrak{A}_{\nu_{1} \cdots \nu_{s}}^{\mu_{1} \cdots \mu_{r}}\left(x^{\alpha}\right) \partial_{\mu_{1}} \otimes \cdots \otimes \partial_{\mu_{r}} \otimes d x^{\nu_{1}} \otimes \cdots \otimes d x^{\nu_{s}} \\
& \otimes(\tau)^{\otimes w}
\end{aligned}
$$

with $\tau:=d x^{0} \wedge \cdots \wedge d x^{3}$. The set of functions

$$
\mathfrak{\mathfrak { A }}_{v_{1} \cdots \nu_{s}}^{\mu_{1} \cdots \mu_{r}}\left(x^{\alpha}\right)=(\sqrt{-\operatorname{det} \mathbf{g}})^{w} A_{\nu_{1} \cdots v_{s}}^{\mu_{1} \cdots \mu_{r}}\left(x^{\alpha}\right)
$$

is said to be the components of the relative tensor field $\mathfrak{U} \in \sec \left(T_{s}^{r} M \otimes\left(\bigwedge^{4} T^{*} M\right)^{w}\right)$ and under a coordinate transformation $x^{\alpha} \mapsto x^{\prime \beta}$ with Jacobian $J=\operatorname{det}\left(\partial x^{\prime \alpha} / \partial x^{\beta}\right)$ these functions transform as $[29,30]$

$$
\begin{aligned}
& \mathfrak{A}_{\kappa_{1} \cdots \kappa_{s}}^{\prime \lambda_{1} \cdots \lambda_{r}}\left(x^{\prime \beta}\right) \\
& \quad=J^{w} \frac{\partial x^{\prime \lambda_{1}}}{\partial x^{\mu_{1}}} \cdots \frac{\partial x^{\prime \lambda_{1}}}{\partial x^{\mu_{1}}} \frac{\partial x^{\nu_{1}}}{\partial x^{\kappa_{1}}} \cdots \frac{\partial x^{\nu_{s}}}{\partial x^{\kappa_{s}}} \mathfrak{P}_{\nu_{1} \cdots \nu_{s}}^{\mu_{1} \cdots \mu_{r}}\left(x^{\alpha}\right) .
\end{aligned}
$$

On a manifold $M$ equipped with a metric tensor field $\mathbf{g}$ we can write $\mathfrak{A}_{v_{1} \cdots \nu_{s}}^{\mu_{1} \cdots \mu_{r}}\left(x^{\alpha}\right)=(\sqrt{-\operatorname{det} \mathbf{g}})^{w} A_{v_{1} \cdots v_{s}}^{\mu_{1} \cdots \mu_{r}}\left(x^{\alpha}\right)$, where $A_{v_{1} \cdots v_{s}}^{\mu_{1} \cdots \mu_{r}}\left(x^{\alpha}\right)$ are the components of a tensor field $A \in \sec T_{s}^{r} M$.

The covariant derivative of a relative tensor field relative to a given arbitrary connection $\nabla$ defined on $M$ such that $\nabla_{\partial / \partial x^{\nu}} d x^{\mu}=-\ell_{\cdot v \alpha}^{\mu \cdot \cdot} d x^{\alpha}$ is given (as the reader may easily find) by

$$
\begin{aligned}
\nabla_{\partial_{\kappa}} \mathfrak{A}= & \left(\nabla_{\kappa} \mathfrak{I}_{v_{1} \cdots v_{s}}^{\mu_{1} \cdots \mu_{r}}\right) \partial_{\mu_{1}} \otimes \cdots \otimes \partial_{\mu_{r}} \otimes d x^{\nu_{1}} \otimes \cdots \\
& \otimes d x^{\nu_{s}} \otimes(\tau)^{\otimes w},
\end{aligned}
$$

where

$$
\begin{aligned}
& \nabla_{\mathcal{K}} \mathfrak{A}_{v_{1} \cdots v_{s}}^{\mu_{1} \cdots \mu_{r}}=\frac{\partial}{\partial x^{\mathcal{K}}} \mathfrak{A}_{v_{1} \cdots \nu_{s}}^{\mu_{1} \cdots \mu_{r}}+\ell_{\cdot \mathcal{L} \mathcal{K}}^{\mu_{p \cdots}} \mathfrak{A}_{v_{1} \cdots \nu_{s}}^{\mu_{1} \cdots \mu_{p-1} \mu \mu_{p+1} \cdots \mu_{r}} \\
& -\ell_{\cdot v_{q} \kappa}^{l \cdot *} \mathfrak{P}_{v_{1} \cdots v_{q-1} \nu_{q+1} \cdots v_{s}}^{\mu_{1} \cdots \mu_{r}}-w \ell_{\cdot \kappa \sigma}^{\sigma \cdot \cdot} \mathfrak{A}_{v_{1} \cdots v_{s}}^{\mu_{1} \cdots \mu_{r}} .
\end{aligned}
$$

In particular for the Levi-Civita connection $\mathbf{D}$ of $\mathbf{g}$ we have for the relative tensor

$$
\tau_{\mathbf{g}}=\sqrt{-\operatorname{det} \mathbf{g}} \otimes d x^{0} \wedge \cdots \wedge d x^{3}
$$

that

$$
\begin{aligned}
\mathbf{D}_{\alpha}(\sqrt{-\operatorname{det} \mathbf{g}}) & =\partial_{\gamma}(\sqrt{\operatorname{det} \mathbf{g}})-\Gamma_{\cdot \gamma \rho}^{\rho \cdot \cdot} \sqrt{\operatorname{det} \mathbf{g}}=0, \\
\mathbf{D}_{\alpha}\left(\frac{1}{\sqrt{-\operatorname{det} \mathbf{g}}}\right) & =\partial_{\gamma}\left(\frac{1}{\sqrt{-\operatorname{det} \mathbf{g}}}\right)+\Gamma_{\cdot \gamma \rho}^{\rho \cdot \cdot} \frac{1}{\sqrt{-\operatorname{det} \mathbf{g}}} \\
& =0 .
\end{aligned}
$$

\section{F. Explicit Formulas for $\mathbf{J}_{\mu \nu}$ and $\mathbf{J}_{\mu 4}$ in Terms of Projective Conformal Coordinates}

We have taking into account (62) the following identities:

$$
\begin{aligned}
\frac{\partial X^{\kappa}}{\partial x^{\alpha}} & =\frac{\Omega^{2}}{2 \ell^{2}} x_{\alpha} x^{\kappa}+\Omega \delta_{\alpha}^{\kappa}, \\
\frac{\partial X^{4}}{\partial x^{\alpha}} & =-\frac{\Omega^{2}}{\ell} x_{\alpha}, \\
X^{\mu} & =\Omega x^{\mu},
\end{aligned}
$$

where $x_{\mu}:=\eta_{\mu \nu} x^{\nu}$ and $X_{\mu}:=\dot{\eta}_{\mu \nu} X^{\nu}$. We want to prove that

$$
\text { (a) } \begin{aligned}
\mathbf{J}_{\mu \nu} & =\eta_{\mu \beta} x^{\beta} \frac{\partial}{\partial x^{\nu}}-\eta_{\nu \beta} x^{\beta} \frac{\partial}{\partial x^{\mu}} \\
& =\stackrel{\circ}{\mu \beta} X^{\beta} \frac{\partial}{\partial X^{\nu}}-\stackrel{\circ}{\nu \beta}_{X^{\beta}} \frac{\partial}{\partial X^{\mu}}, \\
\text { (b) } \mathbf{J}_{\mu 4} & =\ell \frac{\partial}{\partial x^{\mu}}-\frac{1}{4 \ell}\left(2 \eta_{\mu \nu} x^{\nu} x^{\lambda}-\sigma^{2} \delta_{\mu}^{\lambda}\right) \frac{\partial}{\partial x^{\lambda}} \\
& =-X^{4} \frac{\partial}{\partial X^{\mu}}+X_{\mu} \frac{\partial}{\partial X^{4}} .
\end{aligned}
$$

Proof. (a) One has

$$
\begin{aligned}
\mathbf{J}_{\mu \nu}= & \eta_{\mu \beta} x^{\beta} \frac{\partial X^{\kappa}}{\partial x^{\nu}} \frac{\partial}{\partial X^{\kappa}}+\eta_{\mu \beta} x^{\beta} \frac{\partial X^{4}}{\partial x^{\nu}} \frac{\partial}{\partial X^{4}} \\
& -\eta_{\nu \beta} x^{\beta} \frac{\partial X^{\kappa}}{\partial x^{\mu}} \frac{\partial}{\partial X^{\kappa}}-\eta_{\nu \beta} x^{\beta} \frac{\partial X^{4}}{\partial x^{\mu}} \frac{\partial}{\partial X^{4}} \\
= & x_{\mu} \frac{\partial X^{\kappa}}{\partial x^{\nu}} \frac{\partial}{\partial X^{\kappa}}-x_{\nu} \frac{\partial X^{\kappa}}{\partial x^{\mu}} \frac{\partial}{\partial X^{\kappa}}+x_{\mu} \frac{\partial X^{4}}{\partial x^{\nu}} \frac{\partial}{\partial X^{4}} \\
& -x_{\nu} \frac{\partial X^{4}}{\partial x^{\mu}} \frac{\partial}{\partial X^{4}} \\
= & x_{\mu}\left(-\frac{\Omega^{2}}{2 \ell^{2}} x_{\nu} x^{\kappa}+\Omega \delta_{\nu}^{\kappa}\right) \frac{\partial}{\partial X^{\kappa}} \\
& -x_{\nu}\left(-\frac{\Omega^{2}}{2 \ell^{2}} x_{\mu} x^{\kappa}+\Omega \delta_{\mu}^{\kappa}\right) \frac{\partial}{\partial X^{\kappa}} \\
& +\left(\frac{\Omega^{2}}{\ell} x_{\nu} x_{\mu}-\frac{\Omega^{2}}{\ell} x_{\mu} x_{\nu}\right) \frac{\partial}{\partial X^{4}}
\end{aligned}
$$




$$
\begin{aligned}
= & X_{\mu} \frac{\partial}{\partial X^{\nu}}-X_{\nu} \frac{\partial}{\partial X^{\mu}}-\frac{\Omega^{2}}{2 \ell^{2}} x_{\nu} x_{\mu} x^{\kappa} \frac{\partial}{\partial X^{\kappa}} \\
& +\frac{\Omega^{2}}{2 \ell^{2}} x_{\nu} x_{\mu} x^{\kappa} \frac{\partial}{\partial X^{\kappa}}=X_{\mu} \frac{\partial}{\partial X^{\nu}}-X_{\nu} \frac{\partial}{\partial X^{\mu}} \\
= & \stackrel{\circ}{\eta}_{\mu \beta} X^{\beta} \frac{\partial}{\partial X^{\nu}}-\dot{\circ}_{\nu \beta} X^{\beta} \frac{\partial}{\partial X^{\mu}} .
\end{aligned}
$$

(b) One has

$$
\begin{aligned}
& \mathbf{J}_{\mu 4}=\ell \frac{\partial}{\partial x^{\mu}}-\frac{1}{4 \ell}\left(2 \eta_{\mu \nu} x^{\nu} x^{\lambda}-\sigma^{2} \delta_{\mu}^{\lambda}\right) \frac{\partial}{\partial x^{\lambda}} \\
& =\ell \frac{\partial}{\partial x^{\mu}}+\frac{1}{4 \ell} \sigma^{2} \frac{\partial}{\partial x^{\mu}}-\frac{1}{4 \ell} 2 \eta_{\mu \nu} x^{\nu} x^{\lambda} \frac{\partial}{\partial x^{\lambda}} \\
& =-\frac{1}{\Omega} X^{4} \frac{\partial}{\partial x^{\mu}}-\frac{1}{2 \ell} \eta_{\mu \nu} x^{\nu} x^{\lambda} \frac{\partial}{\partial x^{\lambda}} \\
& =-\frac{1}{\Omega} X^{4}\left(\frac{\Omega^{2}}{2 \ell^{2}} x_{\mu} x^{\kappa}+\Omega \delta_{\mu}^{\kappa}\right) \frac{\partial}{\partial X^{\kappa}}-\frac{1}{\Omega} X^{4} \frac{\partial X^{4}}{\partial x^{\mu}} \frac{\partial}{\partial X^{4}} \\
& -\frac{1}{2 \ell} x_{\mu} x^{\lambda}\left(\frac{\Omega^{2}}{2 \ell^{2}} x_{\lambda} x^{\kappa}+\Omega \delta_{\lambda}^{\kappa}\right) \frac{\partial}{\partial X^{\kappa}} \\
& -\frac{1}{2 \ell} \eta_{\mu \nu} x^{\nu} x^{\lambda} \frac{\partial X^{4}}{\partial x^{\lambda}} \frac{\partial}{\partial X^{4}} \\
& =-X^{4} \frac{\partial}{\partial X^{\mu}}-\frac{\Omega}{2 \ell^{2}} X^{4} x_{\mu} x^{\kappa} \frac{\partial}{\partial X^{\kappa}}-\frac{1}{2 \ell} x_{\mu} x^{\lambda} \Omega \frac{\partial}{\partial X^{\lambda}} \\
& -\frac{1}{4 \ell^{3}} \Omega^{2} \sigma^{2} x_{\mu} x^{\kappa} \frac{\partial}{\partial X^{\lambda}}+X^{4} \Omega x_{\mu} \frac{\partial}{\partial X^{4}} \\
& +\frac{1}{2 \ell^{2}} x_{\mu} \Omega^{2} x^{\lambda} \frac{\partial X^{4}}{\partial x^{\lambda}} \frac{\partial}{\partial X^{4}} \\
& =-X^{4} \frac{\partial}{\partial X^{\mu}}+\left\{X^{4} \Omega+\frac{1}{2 \ell^{2}} \Omega^{2} \sigma^{2}\right\} x_{\mu} \frac{\partial}{\partial X^{4}} \\
& -\left\{\frac{1}{\ell} X^{4}+1+\frac{1}{2 \ell^{2}} \Omega \sigma^{2}\right\} \frac{1}{2 \ell} \Omega x_{\mu} x^{\lambda} \frac{\partial}{\partial X^{\lambda}} \\
& =-X^{4} \frac{\partial}{\partial X^{\mu}}+X_{\mu} \frac{\partial}{\partial X^{4}} \\
& -\left\{-\left(1+\frac{\sigma^{2}}{4 \ell^{2}}\right)+\frac{1}{\Omega}+\frac{1}{2 \ell^{2}} \sigma^{2}\right\} \frac{1}{2 \ell} \Omega^{2} x_{\mu} x^{\lambda} \frac{\partial}{\partial X^{\lambda}} \\
& =-X^{4} \frac{\partial}{\partial X^{\mu}}+X_{\mu} \frac{\partial}{\partial X^{4}} \\
& -\left\{-1+\frac{1}{4 \ell^{2}} \sigma^{2}+1-\frac{1}{4 \ell^{2}} \sigma^{2}\right\} \frac{1}{2 \ell} \Omega^{2} x_{\mu} x^{\lambda} \frac{\partial}{\partial X^{\lambda}} \\
& =-X_{4} \frac{\partial}{\partial X^{\mu}}+X_{\mu} \frac{\partial}{\partial X^{4}},
\end{aligned}
$$

where we used the fact that

$$
\begin{aligned}
&\left\{X^{4} \Omega \frac{1}{\ell}+\frac{1}{2 \ell^{2}} \Omega^{2} \sigma^{2}\right\} x_{\mu} \frac{\partial}{\partial X^{4}}=\left\{-\left(1+\frac{1}{4 \ell^{2}} \sigma^{2}\right)+\frac{2}{4 \ell^{2}} \sigma^{2}\right\} \\
& \cdot \Omega^{2} x_{\mu} \frac{\partial}{\partial X^{4}}\left\{-\left(1+\frac{1}{4 \ell^{2}} \sigma^{2}\right)+\frac{2}{4 \ell^{2}} \sigma^{2}\right\} \Omega^{2} x_{\mu} \frac{\partial}{\partial X^{4}} \\
&=-\frac{1}{\Omega} \Omega^{2} x_{\mu} \frac{\partial}{\partial X^{4}}=-X_{\mu} \frac{\partial}{\partial X^{4}} .
\end{aligned}
$$

\section{Competing Interests}

The authors declare that they have no competing interests regarding the publication of this paper.

\section{Endnotes}

1. The energy-momentum covector is an element of a vector space and is not a covector field.

2. This section is an improvement of results first presented in [9].

3. The symbol $\partial$ denotes the Dirac operator acting on sections of the Clifford bundle $\mathscr{C} \ell(M, \mathrm{~g})$. See Appendix A.

4. Not a covector field.

5. Some of the material of the appendices is well known, but we think that despite this fact their presentation here will be useful for most of our readers.

6. The Clifford bundle formalism permits the representation of a covariant Dirac spinor field as certain equivalence classes of even sections of the Clifford bundle, called Dirac-Hestenes spinor field (DHSF). These objects are a key ingredient to clarify the concept of Lie derivative of spinor fields and give meaningful definition for such an object, something necessary to study conservation laws in Lorentzian space-time structures when spinor fields are present. Our approach to the subject is described in [25] and a thoughtful derivation of Dirac equation in de Sitter structure $(M, \mathbf{g})$ using DHSFs is given in [16].

7. See Chapter 4 of [11].

8. The maximum number is 10 when $\operatorname{dim} M=4$ and that maximum number occurs only for space-times of constant curvature.

9. See [11] for the rest of the notation.

10. Keep in mind that in (25) $T_{\alpha \nu}$ are the $\nu$-component of the current $\mathscr{T}_{\alpha}=\mathscr{J}_{\partial / \partial x^{\alpha}}$ and moreover are here taken as symmetric. See Appendix C.3.3.

11. In fact, by an equivalence classes of quintuples $\left(M, \mathbf{g}, \mathbf{D}, \tau_{\mathbf{g}}, \uparrow\right)$ are modulo diffeomorphisms.

12. See however Section 2.4 to learn that this naive expectation is incorrect. 
13. The concept of asymptotically flat Lorentzian manifold can be rigorously formulated without the use of coordinates, as, for example, in [31]. However we will not need to go into detail here.

14. Komar called a related quantity the generalized flux.

15. $V$ denotes a spacelike hypersurface and $S=\partial V$ its boundary. Usually the integral $\mathfrak{E}$ is calculated at a constant $x^{0}$ time hypersurface and the limit is taken for $S$ being the boundary at infinity.

16. Please do not confuse $\boldsymbol{\delta}^{0}$ with $\underset{\mathrm{g}}{\boldsymbol{\delta}}$.

17. $\mathscr{G}^{\mu}=G_{v}^{\mu} \boldsymbol{\vartheta}^{v}$, where $G_{v}^{\mu}=R_{v}^{\mu}-(1 / 2) \delta_{v}^{\mu}$ are the components of the Einstein tensor. Moreover, we write $\mathscr{E}^{\mu}=E_{\nu}^{\mu} \boldsymbol{\vartheta}^{\nu}$.

18. $\mathscr{C} \ell(M, \mathrm{~g})$ is the Clifford bundle of differential forms; see Appendix and if more details are necessary, consult, for example, [11].

19. Note that since $\underset{\mathbf{g}}{\delta}\left(\mathscr{G}^{\mu} A_{\mu}\right)=0$ it follows from (45) that indeed $\underset{\mathbf{g}}{\delta} L=0$.

20. Something that is not given in [6].

21. Recall that in relativity theory (both special and general) a reference frame is modelled by a timelike vector field $\mathbf{Z}$ pointing into the future. A naturally adapted coordinate chart to $\mathbf{Z}$ (with coordinate functions $\left\{x^{\mu}\right\}$ (denoted (nacs $\mid \mathbf{Z})$ )) is one such that the spatial components of $\mathbf{Z}$ are null. More details may be found, for example, in Chapter 6 of [11].

22. An equivalent formula appears, for example, as Equation (11.2.10) in [31]. However, the simplicity and transparency of our approach concerning traditional ones based on classical tensor calculus is to be emphasized here.

23. The total system is the system consisting of the gravitational plus matter and nongravitational fields.

24. A group $G$ of transformations in a manifold $M(\sigma: G \times$ $M \rightarrow M$ by $(g, x) \mapsto \sigma(g, x))$ is said to act transitively on $M$ if for arbitraries $x, y \in M$ there exists $g \in G$ such that $\sigma(g, x)=y$.

25. Figure 1 appears also in author's paper [16].

26. More details are in Section 5.1.

27. We are using here a notation similar to the ones in [1] for comparison of some of our results with the ones obtained there.

28. In (76) $\mathrm{Y}$ is the deformation vector field determining the curves $\sigma(s, l)$ necessary to calculate the first variation of $I[\sigma]$.

29. Take notice that $\Gamma_{\cdot \mu \alpha}^{\beta \cdot \cdot} \neq \Gamma_{\cdot \mu \alpha}^{\beta \cdot \cdot}$, where $D_{\partial_{\mu}} \partial_{\alpha}=\Gamma_{\cdot \mu \alpha}^{\beta \cdot \cdot} \partial_{\beta}$, for otherwise confusion will arise.

30. Proofs of (90) and (91) are in Appendix F. Of course, in those equations, it is $\mathbf{P}_{\alpha}=e_{\alpha}$ (as introduced is Section 2).

31. Recall that the fields $\mathbf{e}_{\alpha}$ are only defined in subset $\left\{S^{3}\right.$-north pole $\}$.
32. The explict form of the $K_{\alpha}^{\kappa}$ can be determined without difficulty if needed.

33. The integration is to be evaluated at a $t=$ const space line hypersurface $\mathcal{S}$.

34. Not to be confused with Minkowski space-time $[4,11]$.

35. Minkowski space-time is the particular case of a Lorentzian space-time structure for which $M \simeq \mathbb{R}^{4}$ and the curvature and torsion tensors of the Levi-Civita connection of Minkowski metric are null. A teleparallel space-time is a particular Riemann-Cartan space-time such that $\mathbf{R}^{\mathbf{D}}=0$ and $\Theta^{\mathbf{D}} \neq 0$.

36. Ad : $\operatorname{Spin}_{1,3}^{e} \rightarrow \operatorname{Aut}\left(\mathbb{R}_{1,3}\right)$ by $\operatorname{Ad}_{u} x=u x u^{-1}$.

37. Take notice that $A d: \operatorname{Spin}_{1,3}^{e} \rightarrow \operatorname{Aut}\left(\mathbb{R}_{1,3}\right)$ such that for any $\mathscr{C} \in \mathbb{R}_{1,3}$ it is $\operatorname{Ad}_{u} \mathscr{C}=u \mathscr{C} u^{-1}$. Since $\operatorname{Ad}_{-1}=\operatorname{Ad}_{1}=$ identity, Ad descends to a representation of $\mathrm{SO}_{1,3}^{e}$ that we denoted by $\mathrm{Ad}^{\prime}$.

38. Also called "spin connection 1-forms."

39. For a general metric compatible Riemann-Cartan connection the formula in (A.22b) is not valid; we have a more general relation involving the torsion tensor that will not be used in this paper. The interested reader may consult [11].

40. For more details concerning the square of Dirac (and spin-Dirac operators) on a general Riemann-Cartan space-time, see [32].

41. Of course, the fields $h^{*} \phi_{1}, \ldots, h^{*} \phi_{N}$ must satisfy deformed initial conditions and deformed boundary conditions.

42. In truth, by matter fields, we understand fields of two kinds, fermion fields (electrons, neutrinos, and quarks) and boson fields (electromagnetic, gravitational, weak, and strong fields).

43. A rigorous formulation needs the introduction of jet bundles (see, e.g., [33]). We will not need such sophistication for the goals of this paper.

44. In [11] the horizontal variation is denoted by $\boldsymbol{\delta}_{\mathrm{h}}$, where a vertical variation denoted by $\boldsymbol{\delta}_{\mathbf{v}}$ (associated with gauge transformations) is also introduced. Moreover, let us recall that $\boldsymbol{\delta}^{0}$ has been used extensively after a famous paper by Rosenfeld [34] but appears also to the best of our knowledge in Section 23 of Pauli's book [35] on relativity theory.

45. As, for example, in [36].

46. Some authors call $\delta \phi_{\mu}(x)$ the total variation, but we think that this is not an appropriate name.

47. For example, in [7], the condition is fixed in such a way that the orbital angular momentum tensor of the system defined as $\mathbf{M}=\left.\left.(1 / 2) M_{\mu \nu} d x^{\mu}\right|_{o} \wedge d x^{\nu}\right|_{o}$ (with $\left.M^{\mu \nu}=\int\left(x^{\mu} T^{\nu \kappa}-x^{\nu} T^{\mu \kappa}\right) d \sigma_{\kappa}\right)$ is automatically conserved. However take into account that since the fields possess in general intrinsic spin an angular momentum conservation law can only be formulated by taking into account the orbital and spin angular momenta. It can be shown 
(see Chapter 8 of [11]) that the antisymmetric part of the canonical energy-momentum tensor is the source of the spin tensor of the field. In [37] how to obtain a conserved symmetrical energy-momentum tensor by studying the conservation laws that come from a general Poincaré variation is shown, which involves translations and general Lorentz transformations.

48. The number $w$ is an integer. Of course, if $w=0$ we are back to tensor fields.

49. The notation $\left(\bigwedge^{4} T^{*} M\right)^{\otimes w}$ means the $w$-fold tensor product of $\wedge^{4} T^{*} M$ with itself.

\section{References}

[1] J. G. Pereira and A. C. Sampson, "De Sitter geodesics: reappraising the notion of motion," General Relativity and Gravitation, vol. 44, no. 5, pp. 1299-1308, 2012.

[2] A. Papapetrou, "Spinning test-particles in general relativity I," Proceedings of the Royal Society of London A, vol. 209, pp. 248225, 1951.

[3] I. M. Benn and R. W. Tucker, An Introduction to Spinors and Geometry with Applications in Physics, Adam Hilger, Bristol, UK, 1987.

[4] R. K. Sachs and H. H. Wu, General Relativity for Mathematicians, Springer, Berlin, Germany, 1977.

[5] W. A. Rodrigues Jr., "The nature of gravitational field and its legitimate energy-momentum tensor," Reports on Mathematical Physics, vol. 69, no. 2, pp. 265-279, 2012.

[6] A. Komar, "Covariant conservation laws in general relativity," Physical Review, vol. 113, no. 3, pp. 934-936, 1959.

[7] L. Landau and E. M. Lifshitz, The Classical Theory of Fields, Pergamon Press, New York, NY, USA, 4th edition, 1975.

[8] P. G. Bergmann, "Conservation laws in general relativity as the generators of coordinate transformations," Physical Review, vol. 112, no. 1, pp. 287-289, 1958.

[9] F. G. Rodrigues, W. A. Rodrigues Jr., and R. Rocha, "The Maxwell and Navier-Stokes equations that follow from Einstein equation in a spacetime containing a Killing vector field," in Proceedings of the 6th International School on Field Theory and Gravitation, W. A. Jr. Rodrigues, R. Kerner, G. O. Pires, and C. Pinheiro, Eds., vol. 1483 of AIP Conference Proceedings, pp. 277295, Rio de Janeiro, Brazil, April 2012.

[10] W. A. Rodrigues Jr., "Killing vector fields, maxwell equations and lorentzian spacetimes," Advances in Applied Clifford Algebras, vol. 20, no. 3, pp. 871-884, 2010.

[11] W. A. Rodrigues Jr. and E. Capelas de Oliveira, The Many Faces of Maxwell, Dirac and Einstein Equations: A Clifford Bundle Approach, vol. 922 of Lecture Notes in Physics, Springer, Berlin, Germany, 2016.

[12] W. A. Rodrigues Jr. and S. A. Wainer, "A Clifford bundle approach to the differential geometry of branes," Advances in Applied Clifford Algebras, vol. 24, no. 3, pp. 817-847, 2014.

[13] S. W. Hawking and G. F. R. Ellis, The Large Scale Structure of Space-Time, Cambridge University Press, Cambridge, UK, 1973.

[14] H.-J. Schmidt, "On the de Sitter space-time-the geometric foundation of inflationary cosmology," Fortschritte der Physik/Progress of Physics, vol. 41, no. 3, pp. 179-199, 1933.

[15] B. O’Neill, Semi-Riemannian Geometry, Academic Press, New York, NY, USA, 1893.
[16] W. A. Rodrigues Jr., S. A. Wainer, M. Rivera-Tapia, E. A. NotteCuello, and I. Kondrashuk, "A Clifford bundle approach to the wave equation of a spin 1/2 fermion in the de Sitter manifold," Advances in Applied Cliford Algebras, vol. 26, no. 3, pp. 253-277, 2015.

[17] F. Gürsey, "Introduction to group theory," in Relativity, Groups and Topology, C. De Witt and B. De Witt, Eds., Gordon and Breach, New York, NY, USA, 1963.

[18] V. C. de Andrade, L. C. Guillen, and J. G. Pereira, "Gravitational energy-momentum density in teleparallel gravity," Physical Review Letters, vol. 84, article 4533, 2000.

[19] V. C. de Andrade, L. C. T. Guillen, and J. G. Pereira, “Teleparallel gravity: an overview," in Proceedings of IX Marcel Grosamman Metting on General Relativity, V. G. Gurzadyar, R. T. Jansen, and R. Ruffini, Eds., World Scientific Publishing, Singapore, 2002, http://arxiv.org/abs/gr-qc/0011087.

[20] J. W. Maluf, "The teleparallel equivalent of general relativity," Annalen der Physik, vol. 525, no. 5, pp. 339-357, 2013.

[21] R. Aldrovandi and J. G. Perira, Teleparallel Gravity: An Introduction, vol. 173 of Fundamental Theories of Physics, Springer, Dordrecht, The Netherlands, 2013.

[22] H. B. Jr. Lawson and M.-L. Michelsohn, Spin Geometry, Princeton University Press, Princeton, NJ, USA, 1989.

[23] R. A. Mosna and W. A. Rodrigues Jr., "The bundles of algebraic and Dirac-Hestenes spinor fields," Journal of Mathematical Physics, vol. 45, no. 7, pp. 2945-2966, 2004.

[24] W. A. Rodrigues Jr., "Algebraic and Dirac-Hestenes spinors and spinor fields," Journal of Mathematical Physics, vol. 45, pp. 29082994, 2004.

[25] R. F. Leão, W. A. Rodrigues Jr., and S. A. Wainer, "Concept of lie derivative of spinor fields. A geometric motivated approach," Advances in Applied Clifford Algebras, 2015.

[26] P. Roman, Introduction to Quantum Field Theory, John Wiley \& Sons, New York, NY, USA, 1969.

[27] B. O'Neill, Elementary Differential Geometry, Academic Press, New York, NY, USA, 1966.

[28] S. Weinberg, Gravitation and Cosmology; Principles and Applications of the General Theory of Relativity, John Wiley \& Sons, New York, NY, USA, 1972.

[29] D. Lovelok and H. Rund, Tensors, Differential Forms, and Variational Principles, John Wiley \& Sons, New York, NY, USA, 1975.

[30] C. Tiee, "Contravariance, Covariance, Densities, and all That: An Informal Discussion on Tensor Calculus," 2006, http://ccom .ucsd.edu/ ctiee/notes/tensors.pdf.

[31] R. M. Wald, General Relativity, University of Chicago Press, Chicago, Ill, USA, 1984.

[32] E. A. Notte-Cuello, W. A. Rodrigues Jr., and Q. A. G. de Souza, "The squares of the Dirac and spin-Dirac operators on a Riemann-Cartan space(time)," Reports on Mathematical Physics, vol. 60, no. 1, pp. 135-157, 2007.

[33] L. Fatibene and M. Francaviglia, Natural and Gauge Natural Formalism for Classical Field Theories, Kluwer Academic publishers, Dordrecht, The Netherlands, 2003.

[34] L. Rosenfeld, "Sur le tenseur d'impulsion-energie," Memoires de l'Académie Royale de Belgique, vol. 18, pp. 1-30, 1940.

[35] W. Pauli, Theory of Relativity, Dover Publications, New York, NY, USA, 1981, Originally Published in German: Relativitätstheorie, Encyclopädie der Mathematischen Wissenschaften.19, B.G., Teubner, Leipzig, 1921. 
[36] A. Trautman, "Foundations and current problems in general relativity," in Lectures on General Relativity, Brandeis Summer Institute in Theoretical Physics 1964, S. Deser and K. W. Ford, Eds., Prentice Hall, Englewood Cliffs, NJ, USA, 1965.

[37] A. O. Barut, Electrodynamics and Classical Theory of Fields and Particles, Dover Publications, New York, NY, USA, 1980. 


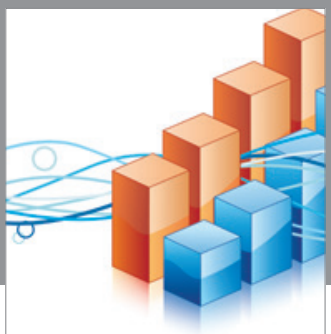

Advances in

Operations Research

vatem alat4

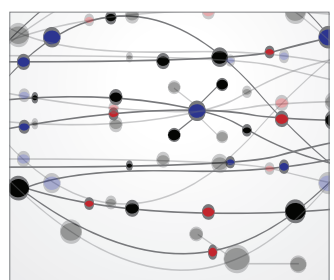

\section{The Scientific} World Journal
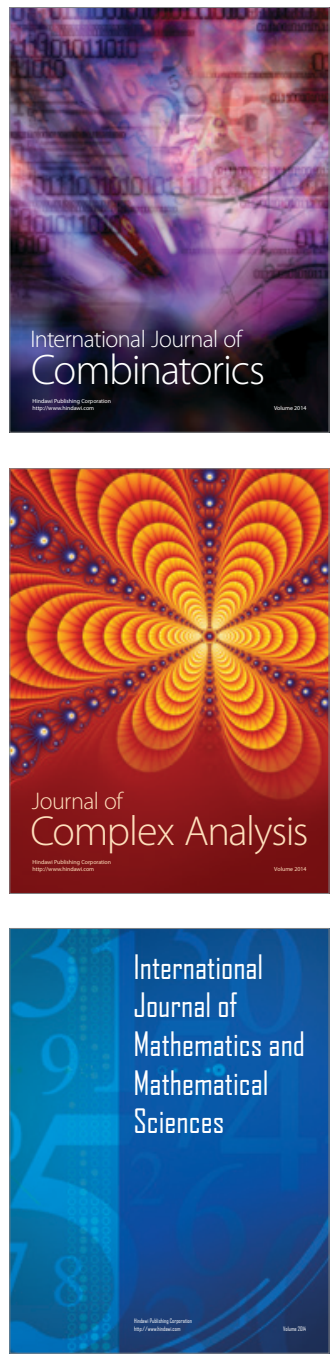
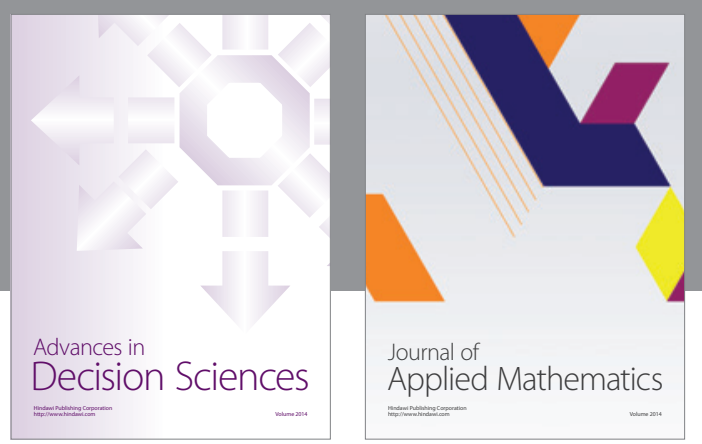

Algebra

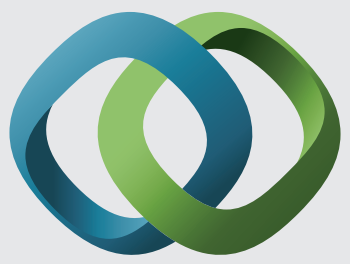

\section{Hindawi}

Submit your manuscripts at

http://www.hindawi.com
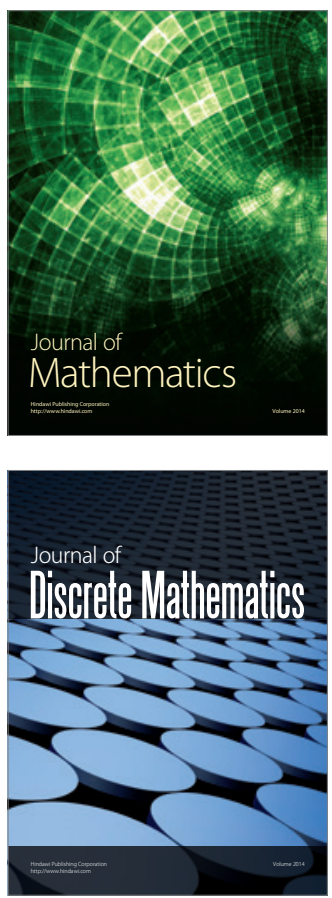

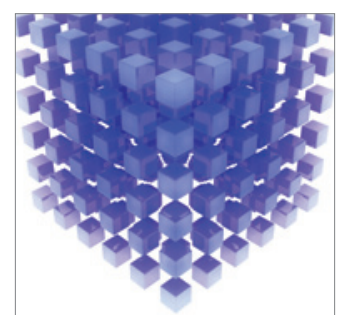

Mathematical Problems in Engineering
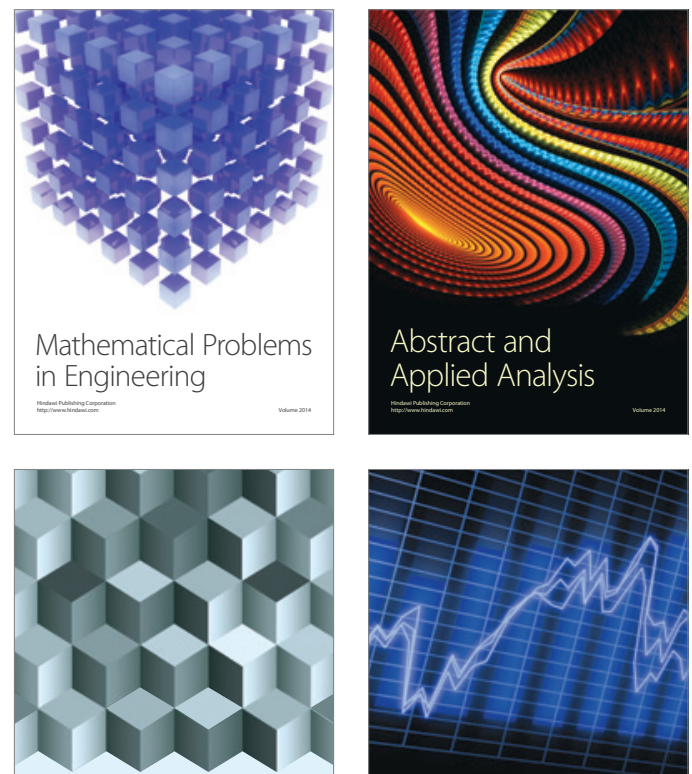

Journal of

Function Spaces

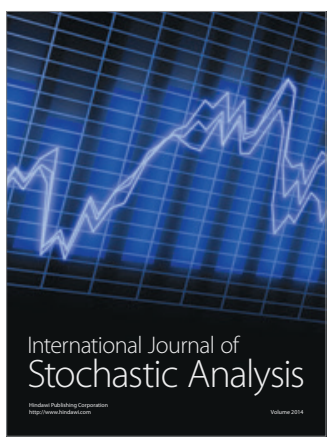

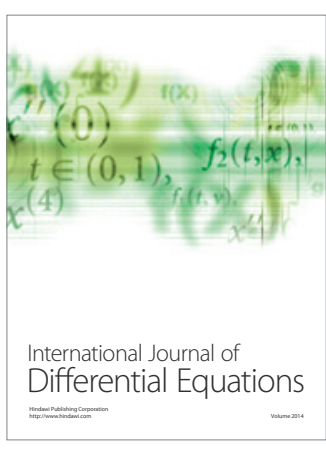
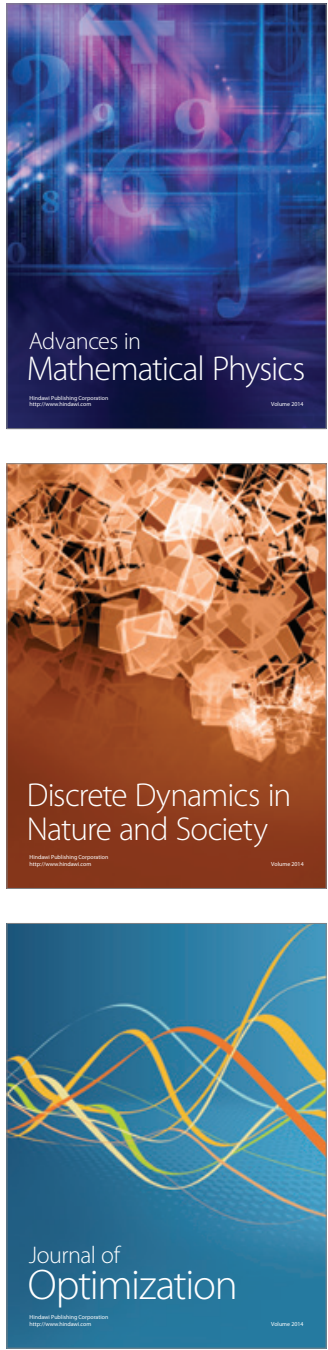